Florida International University

FIU Digital Commons

FIU Electronic Theses and Dissertations

University Graduate School

7-3-2019

\title{
Towards Stable Electrochemical Sensing for Wearable Wound Monitoring
}

Sohini RoyChoudhury

Florida International University, sroyc003@fiu.edu

Follow this and additional works at: https://digitalcommons.fiu.edu/etd

Part of the Biomedical Commons, Enzymes and Coenzymes Commons, Investigative Techniques

Commons, Medical Biochemistry Commons, Nanoscience and Nanotechnology Commons, Nanotechnology Fabrication Commons, Polymer and Organic Materials Commons, Skin and Connective

Tissue Diseases Commons, Therapeutics Commons, and the Translational Medical Research Commons

\section{Recommended Citation}

RoyChoudhury, Sohini, "Towards Stable Electrochemical Sensing for Wearable Wound Monitoring" (2019). FIU Electronic Theses and Dissertations. 4217.

https://digitalcommons.fiu.edu/etd/4217

This work is brought to you for free and open access by the University Graduate School at FIU Digital Commons. It has been accepted for inclusion in FIU Electronic Theses and Dissertations by an authorized administrator of FIU Digital Commons. For more information, please contact dcc@fiu.edu. 


\section{FLORIDA INTERNATIONAL UNIVERSITY}

Miami, Florida

\section{TOWARD STABLE ELECTROCHEMICAL SENSING FOR WEARABLE WOUND MONITORING}

A dissertation submitted in partial fulfillment of

the requirements for the degree of

DOCTOR OF PHILOSOPHY

in

ELECTRICAL ENGINEERING

by

Sohini RoyChoudhury 
To: Dean John L. Volakis

College of Engineering and Computing

This dissertation, written by Sohini RoyChoudhury, and entitled Toward Stable Electrochemical Sensing for Wearable Wound Monitoring, having been approved in respect to style and intellectual content, is referred to you for judgment.

We have read this dissertation and recommend that it be approved.

Robert S. Kirsner

Veena Misra

Michael Daniele

Kingsley Lau

Shubhendu Bhardwaj

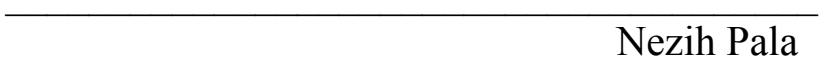

Shekhar Bhansali, Major Professor

Date of Defense: July 3, 2019.

The dissertation of Sohini RoyChoudhury is approved.

Dean John L. Volakis College of Engineering and Computing

Andrés G. Gil

Vice President for Research and Economic Development and Dean of the University Graduate School

Florida International University, 2019 
(C) Copyright 2019 by Sohini RoyChoudhury

All rights reserved. 


\section{DEDICATION}

To my parents, Mr. Somnath RoyChoudhury and Dr. Devjani RoyChoudhury (PhD); my grandparents, Late Dr. Birendra Nath Bose (PhD/DSc)

and Late Mrs. Anima RoyChoudhury;

my little brother Aditya and beloved pet Twixy 


\section{ACKNOWLEDGMENTS}

First and foremost, I would like to express sincere and deep gratitude to my major professor, Dr. Shekhar Bhansali, for having given me the opportunity to undertake this study for my doctoral dissertation. I am indebted to him for his invaluable guidance and support during this journey. His encouragement, motivation and mentorship have been instrumental in enriching my research skills and personality through several opportunities provided to me during my time at Florida International University (FIU).

A big thank you to my committee members Dr. Nezih Pala, Dr. Shubhendu Bhardwaj, Dr. Kingsley Lau, Dr. Veena Misra, Dr. Michael Daniele and Dr. Robert Kirsner for their valuable inputs provided to me from time to time. It is also a pleasure to extend my sincere gratitude to Dr. Misra and Dr. Daniele, for agreeing to be on my committee, offering guidance and advice on my research at multiple conferences, seminars and networking events. Special mention of Dr. Kirsner is to be made for accepting the invitation to be on my dissertation committee and Dr. Hadar Lev-Tov for his valuable suggestions during this study.

A special thank you is due to Dr. Yogeswaran Umasankar for his constant guidance and encouragement during the entire tenure of my research study. I would also like to take this opportunity to thank Dr. Patrick Roman and Dr. Mubarak Mujawar for their advice and suggestions whenever it was required. My profound gratitude is also due to all the deft engineers and staff of Advanced Materials and Research Institute (AMERI) at FIU for maintaining the laboratory facilities and providing me with the necessary tools for my research work. I would like to also convey my thanks to Dr. Munroe, Dr. Hutcheson and Dr. Andriy, for allowing me to use their laboratory equipment in my study. 
I am immensely grateful to the staff of the Department of Electrical and Computer Engineering, FIU and in particular Ms. Pat Brammer, Ms. Luisa Ruiz, Ms. Layla El-Hilu, Ms. Xiang Li and Ms. Mais Kayali for all their administrative support. In addition, special acknowledgement is due to the Wound Clinic at University of Miami and funding sources from NSF NERC - ASSIST, which made this work possible.

My sincere thanks go to Dr. Aparajita Singh, Dr. Syed Khalid Pasha, Dr. Ahmed Hasnain Jalal, Dr. Pandiaraj Manickam, Michelle, Krystine and Kevin, my former colleagues and members of BioMEMS and Microsystems Lab at FIU whose co-operation has helped me in each step of my work. I would also like to express appreciation to my colleagues Apurva, Pulak, Lamar and Randy for their support. My acknowledgements would remain incomplete without mentioning my friends Fahmida, Kajal, Khushbu, Wardalynn, Vanora, Ester and Malak, without whom my days at FIU would not have been as enjoyable.

Finally, I am extremely grateful to my family for having faith in me and being a constant source of motivation and support at all times. I am forever indebted to my parents for their unyielding love, guidance, encouragement and support throughout my research journey and all pursuits. 


\title{
ABSTRACT OF THE DISSERTATION \\ TOWARD STABLE ELECTROCHEMICAL SENSING FOR WEARABLE WOUND MONITORING
}

\author{
by \\ Sohini RoyChoudhury \\ Florida International University, 2019 \\ Miami, Florida

\section{Professor Shekhar Bhansali, Major Professor}

Wearable biosensing has the tremendous advantage of providing point-of-care diagnosis and convenient therapy. In this research, methods to stabilize the electrochemical sensing response from detection of target biomolecules, Uric Acid (UA) and Xanthine, closely linked to wound healing, have been investigated. Different kinds of materials have been explored to address such detection from a wearable, healing platform. Electrochemical sensing modalities have been implemented in the detection of purine metabolites, UA and Xanthine, in the physiologically relevant ranges of the respective biomarkers. A correlation can be drawn between the concentrations of these bio-analytes and wound severity, thus offering probable quantitative insights on wound healing progression. These insights attempt to contribute in reducing some impacts of the financial structure on the healthcare economy associated with wound-care.

An enzymatic electrochemical sensing system was designed to provide quick response at a cost-effective, miniaturized scale. Robust enzyme immobilization protocols have assisted in preserving enzyme activity to offer stable response under relevant variations of temperature and $\mathrm{pH}$, from normal. Increased hydrophilicity of the sensor 
surface using corona plasma, has assisted in improving conductivity, thus allowing for increased electroactive functionalization and loading across the substrate's surface. Superior sensor response was attained from higher loading of nanomaterials (MWCNT/AuNP) and enzymes (UOx/XO) employed in detection.

Potentiometric analyses of the nanomaterial modified enzymatic biosensors were conducted using cyclic voltammetry (CV) and differential pulse voltammetry (DPV) modalities. Under relevant physiological conditions, the biosensor was noted to offer a variation in response between $10 \%$ and $30 \%$ within a week. Stable, reproducible results were obtained from repeated use of the biosensor over multiple days, also offering promise for continuous monitoring. Shelf life of the biosensor was noted to be more than two days with response retained by about $80 \%$ thereafter. Secondary analyses have been performed utilizing the enzymatic biosensor to explore the feasibility of target biomarker detection from clinical extracts of different biofluids for wound monitoring. Biosensor response evaluation from the extracts of human wound exudate, and those obtained from perilesional and healthy skin, provided an average recovery between $107 \%$ and $110 \%$ with a deviation within (+/-) $6 \%$. Gradual decrease in response (10-20\%) was noted in detection from extracts further away from injury site. Increased accumulation of biofluids on the sensor surface was studied to explore sensor response stability as a function of sample volume. With a broad linear range of detection $(0.1 \mathrm{nM}-7.3 \mathrm{mM})$ and detection limits lower than the physiological concentrations, this study has assessed the viability of stable wound monitoring under physiologically relevant conditions on a wearable platform. 


\section{TABLE OF CONTENTS}

\section{CHAPTER}

PAGE

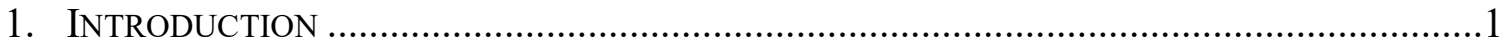

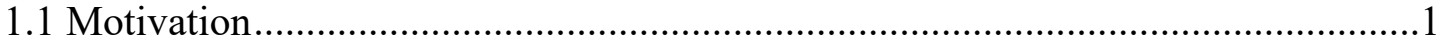

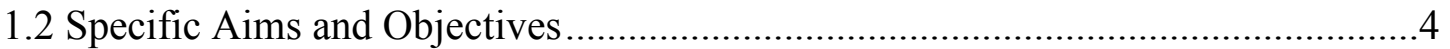

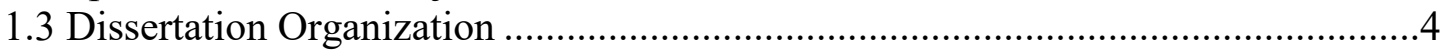

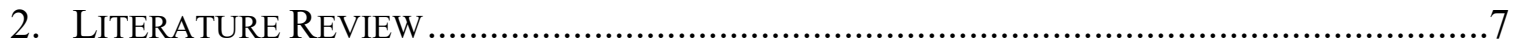

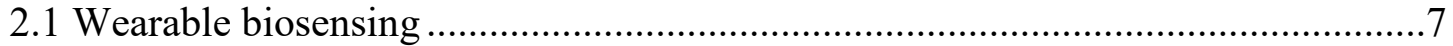

2.1.1 Current state-of-the-art in wearable biosensing ....................................... 7

2.1.2 Detection mechanisms explored in wearable biosensing...........................8

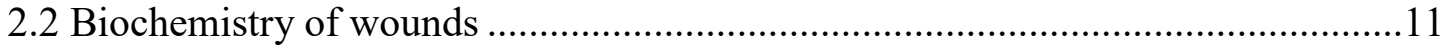

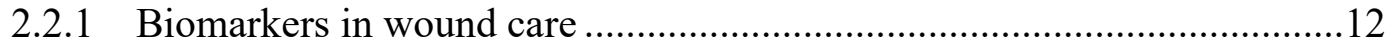

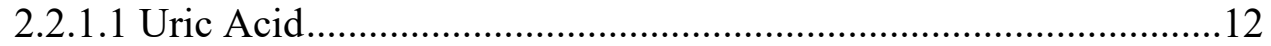

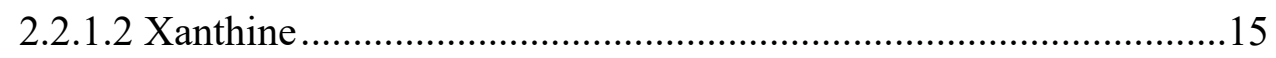

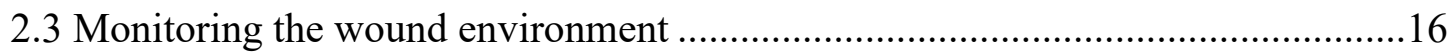

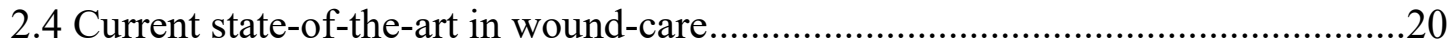

2.5 Mechanisms explored in detection of wound biomarkers ................................21

2.6 Electrochemical determination of wound biomarkers ........................................24

2.7 Nanomaterials employed in electrochemical detection of wound biomarkers ......25

2.8 Substrate materials explored in wearable biosensing ......................................28

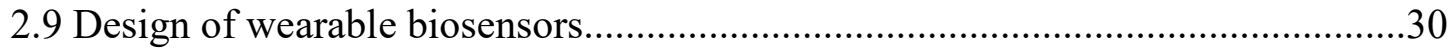

3. ENZYMATIC BIOSENSOR FOR STABLE DETECTION OF URIC ACID BY ENTRAPMENT IN

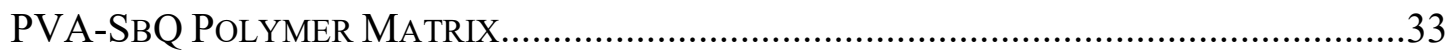

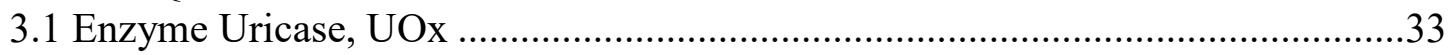

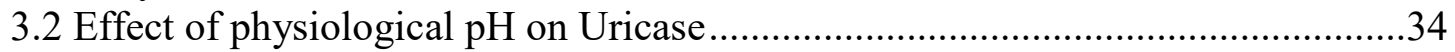

3.3 Enzyme immobilization protocols in detection of Uric Acid ..............................35

3.3.1 Uricase entrapment in PVA-SbQ polymer matrix ................................36

3.4 Ferrocene Carboxylic Acid as mediator in Uric Acid detection.............................37

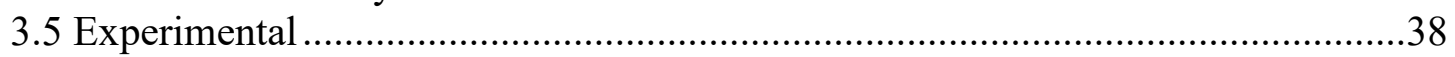

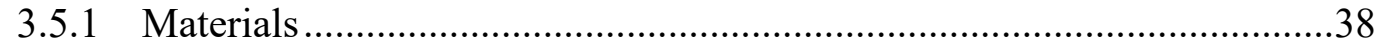

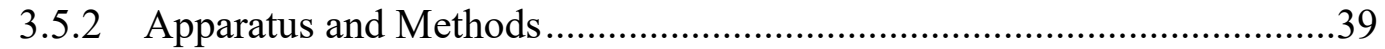

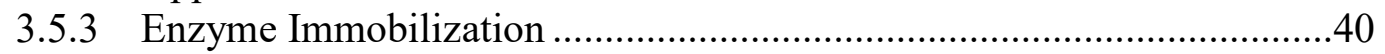

3.6 Entrapped enzymatic biosensor surface characterization .................................40

3.7 Reaction mechanism in enzymatic Uric Acid detection ...................................49

3.8 Electrochemical characterization of entrapped enzymatic biosensor ...................53

3.9 Continuous monitoring and stability at physiologically relevant temperatures.....56

3.10 Selectivity from major electroactive interferents..........................................59

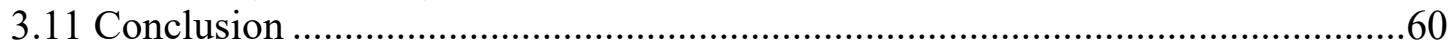


4. Nanocomposite EnZymatic Sensor for Monitoring Xanthine in Wound

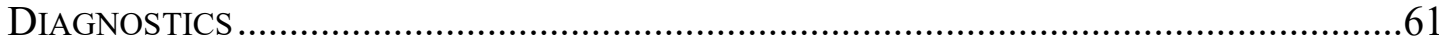

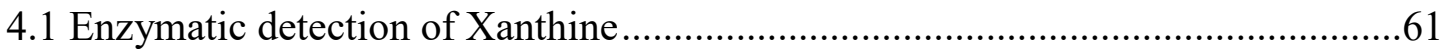

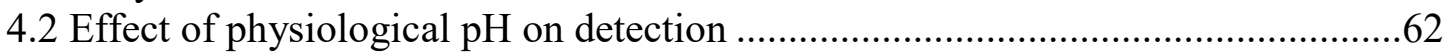

4.3 Use of nanomaterial in electrochemical detection ...........................................63

4.3.1 MWCNT/AuNP nanocomposite in detection of Xanthine ......................63

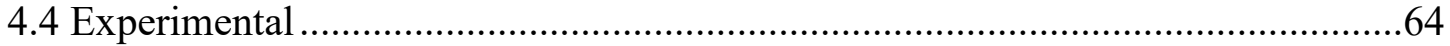

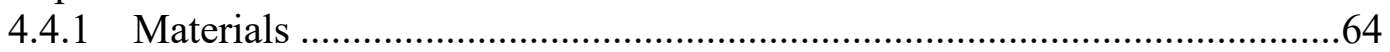

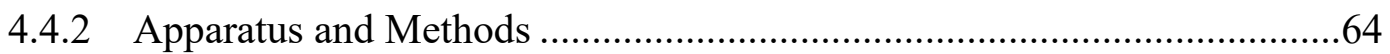

4.4.3 Electrode functionalization .................................................................66

4.5 MWCNT/AuNP nanocomposite sensor surface characterization.......................67

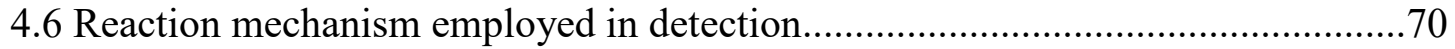

4.7 Sensing performance from MWCNT/AuNP nanocomposite ............................... 71

4.8 Bi-enzymatic performance using MWCNT/AuNP nanocomposite .......................73

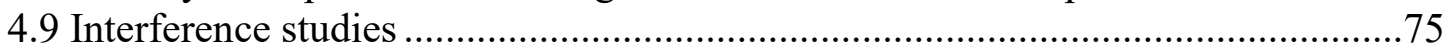

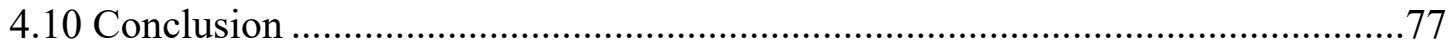

5. DESIGN OF BIOSENSOR FOR WEARABLE WOUND MONITORING AND CLINICAL SAMPLE

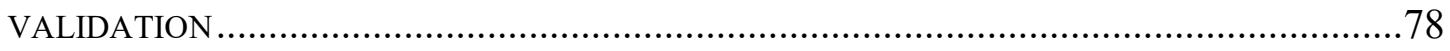

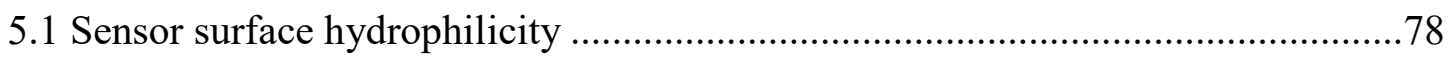

5.2 Detection of wound biomarker from wearable construct ...................................8

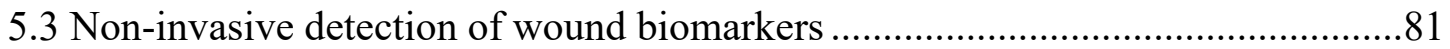

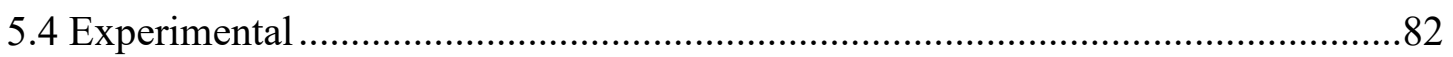

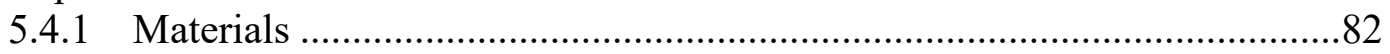

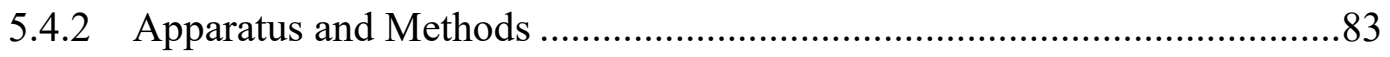

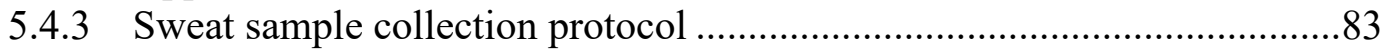

5.4.4 Analyte extraction from wound dressing ........................................... 85

5.4.5 Electrode fabrication on wearable construct...........................................86

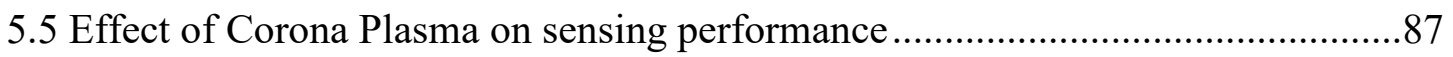

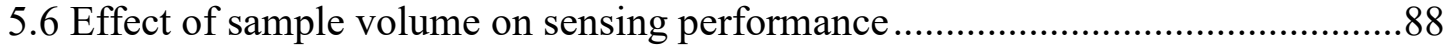

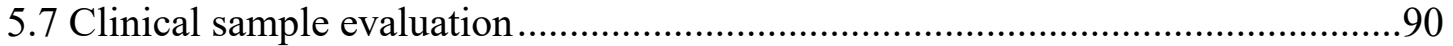

5.7.1 Measurement of UA in sweat samples..................................................90

5.7.2 Measurement of UA in extracted wound exudate...................................90

5.7.3 Effect of sweat lipid emulsification on sensor signal ............................92

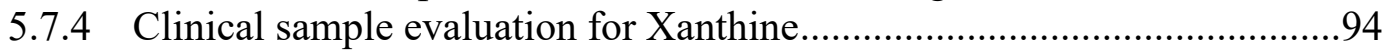

5.7.5 Effect of biofluid occlusion on sensor surface ....................................96

5.8 Electrochemical sensing performance on wearable platform .............................97

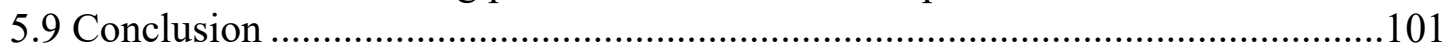

6. SUMMARY AND FUtURE DiRECTION ................................................................ 102

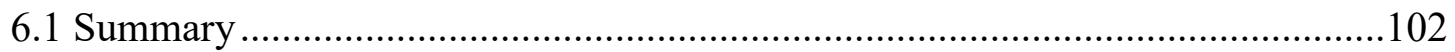

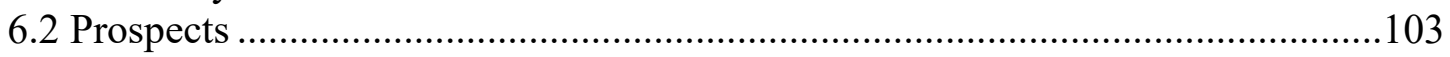

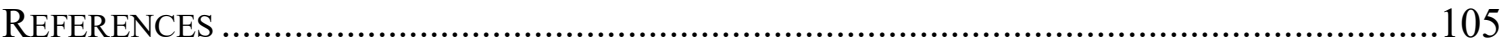

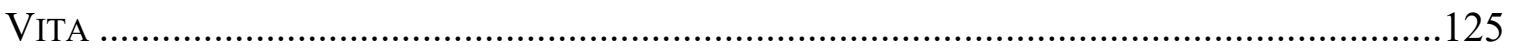




\section{LIST OF TABLES}

TABLE

PAGE

2.1 Comparison of conventional real time wound-care biosensors

2.2 Comparison of materials employed in enzymatic Uric acid determination using UOx functionalized electrochemical biosensors. RT represents room temperature

4.1 Response of sensor for $3 \mu \mathrm{M}$ xanthine in absence and presence of interfering biomolecules

5.1 Colorimetric UA assay vs. electrochemical detection of UA in human sweat samples using an enzymatic biosensor

5.2 Colorimetric UA assay vs. electrochemical detection of UA in extracted wound fluid of de-identified patients using an enzymatic biosensor ...................... 91

5.3 Detection of xanthine in extracted wound exudate, biofluid from perilesional and healthy skin of de-identified patients using the biosensor 


\section{LIST OF FIGURES}

\section{FIGURE}

2.1 (a) TactArray, flexible capacitive array tactile sensor from Pressure Profile Systems, (b) Flexible, wearable disposable cardiac biosensor for remote and ambulatory monitoring; (c) Wearable sensor array for multiplexed in situ perspiration analysis; (d) Tattoo biosensor for monitoring electrolytes from sweat

2.3 Chemical structure of biomolecules (a) Uric Acid (b) Xanthine

2.4 Correlation of Uric Acid concentrations with wound healing 14

2.5 Existing protocols in wound management offering qualitative assessment from (a) visual inspection, (b) wound imaging; and (c) wound mapping; (d) $\mathrm{pH}$ monitor from Empa Inc. for tracking healing of wounds; (e) Wound moisture monitor from WoundSense

3.1 Comparison of $\mathrm{pH}$ effect on specific activity of UOx and current density of UOx electrode

3.2 (a) PVA-SbQ interaction with negatively charged amino acid chains of UOx enzyme, (b) Inset depicts structural interactions between the carboxylic functional group of the enzyme and the amine side chains of the polymer

3.3 Plot representing stability of the pristine PVA-SbQ obtained from continuous measurements for $n=4$ over multiple days with RSD of 0.41

3.4 Raman spectra of UOx functionalized substrates measured with $514.5 \mathrm{~nm}$ Ar laser. (a) Polymer-UOx ratio 1:2 in (-) red line, 1:4 in (- - ) dashed purple line, 1:6 in (-) green line, UOx physisorbed in $(-)$ dark blue line and pristine polymer in (-) purple line. The red, purple, green, blue bands represent the groups in PVA-SbQ. (b) amide III (red band) and (c) phenylalanine (green band) groups in polymer-UOx

3.5 Raman spectra of UOx functionalized substrate measured with $514.5 \mathrm{~nm}$ Ar laser, showing peaks of amino acid residues depicting bonds of (a) C-C (yellow band) and (b) $\mathrm{CO}_{2}^{-}$(orange band) in Arginine; (c) $\mathrm{C}=\mathrm{C}$ and $-\mathrm{CH}_{3}$ in Valine (green band); and (d) $-\mathrm{CO}_{2}{ }^{-}$(blue band) in Leucine. The dashed purple line is the signal from pristine $\mathrm{PVA}-\mathrm{SbQ}$ 
3.6 Fluorescence microscopic images of UOx functionalized substrates with various polymer-UOx ratios of (a) 1:2; (b) 1:4; (c) 1:6; Arrows indicate vacant spaces from reduced loading and low distribution of enzyme across sensor surface; and (d) physisorbed; (e) depicts comparison of mean intensities emitted at $570 \mathrm{~nm}$ from the UOx substrates

3.7. Plot depicting comparison of fluorescence intensities emitted from different UOx loaded substrates at $570 \mathrm{~nm}$. The mean pixel intensities of 1:2, 1:4 and 1:6 polymer-UOx substrates were $1830.89,742.64$ and 440.96 (a.u.) respectively

3.8 SEM micrographs of UOx (a) physisorbed and (b) entrapped functionalized substrates at $100 \mathrm{~nm}$

3.9 (a) Nyquist plot showing variation of impedance in presence of FCA at $0.3 \mathrm{~V}$ between $1 \mathrm{mHz}$ to $100 \mathrm{kHz}$ on functionalized substrates with varied loading; (b) Plot depicting change in UOx activity and current density with varied UOx loading 46

3.10 Bode plot showing variation in impedance (a) phase angle and (b) magnitude on different UOx loaded substrates with frequency from $0.001 \mathrm{~Hz}$ to $100 \mathrm{kHz}$ at at $0.3 \mathrm{~V}$

3.11 Schematic representation of uric acid oxidation by UOx and oxidation of $\mathrm{H}_{2} \mathrm{O}_{2}$ via mediated electron transfer using FCA as redox shuttle

3.12 (a) CV response of UOx entrapped electrode in presence of $20 \mathrm{mM} \mathrm{FCA}$, showing Cycles 1 and 2 with oxidation peak potential, $E \mathrm{p}_{\mathrm{a}}=0.4 \mathrm{~V}$, (b) Reaction mechanism depicting inhibition of $\mathrm{O}_{2}$ in its absence and presence; and its effect on FCA signal $\left(E \mathrm{p}_{\mathrm{a}}=0.45 \mathrm{~V}\right)$, (c) Electron transfer mechanism depicting inhibition of $1 \mathrm{mM} \mathrm{H}_{2} \mathrm{O}_{2}$ in the absence and presence of HRP; and its effect on FCA signal, (d) Concentration of $\mathrm{H}_{2} \mathrm{O}_{2}$ in presence and absence $\mathrm{FCA}$, measured by $\mathrm{H}_{2} \mathrm{O}_{2}$ assay. All the above $\mathrm{CV}$ measurements were carried out at $20 \mathrm{mV} \mathrm{s}^{-1}$ 51

3.13 CV response of UOx entrapped electrode in presence and absence of $20 \mathrm{mM}$ FCA to $0,0.3,1$, and $50 \mathrm{mM} \mathrm{H}_{2} \mathrm{O}_{2}$ at a scan rate of $20 \mathrm{mV} \mathrm{s}^{-1}$. This reveals that FCA acts as an electrochemical mediator for $\mathrm{H}_{2} \mathrm{O}_{2}$ oxidation

3.14 (a) $\mathrm{CV}$ response of $48 \mu \mathrm{M}$ uric acid on bare, only polymer, UOx physisorbed and entrapped electrodes in presence and absence of $20 \mathrm{mM}$ FCA at the scan rate of $20 \mathrm{mV} \mathrm{s}^{-1}$. The inset is the plot of $I \mathrm{p}_{\mathrm{a}} \mathrm{vs}$. uric acid concentration in physiological range $(12-100 \mu \mathrm{M})$. (b) DPV response of $48 \mu \mathrm{M}$ uric acid on UOx physisorbed and entrapped electrodes at the amplitude $50 \mathrm{mV}$ in presence of $20 \mathrm{mM}$ FCA. In the inset, the continuous line represents entrapped electrode and the dotted line represents physisorbed 
3.15. CV response of UOx entrapped electrode in presence and absence of UA at a scan rate of $20 \mathrm{mV} \mathrm{s}^{-1}$

3.16 (a) Plot representing continuous measurements of the UOx electrode over $30 \mathrm{~min}$ in $48 \mathrm{mM}$ uric acid. (b) Plot representing shelf life of the UOx electrodes, where the measurements were carried out in $48 \mu \mathrm{M}$ uric acid

3.17 (a) Plot representing repeatability of the UOx electrode obtained through repeated measurements of $48 \mu \mathrm{M}$ uric acid over a week; (b) Distribution curve representing reproducibility obtained from nine different UOx electrodes measured at the same condition

3.18 (a) Amperometric signal of UA $(100 \mu \mathrm{M})$ in the absence and presence of ascorbic acid $(100 \mu \mathrm{M})$ and dopamine $(100 \mu \mathrm{M})$. (b) Chart representing the reduction in current in presence and absence of ascorbic acid and dopamine at $20 \mathrm{~s}$

4.1 Effect of $\mathrm{pH}$ on the current and potential of the bi-enzymatic sensor in presence of xanthine functionalization in presence of Xanthine

4.2 Plot depicting change in response with varied MWCNT/AuNP and XO/HRP functionalization in presence of Xanthine

4.3 SEM images of (a) MWCNT, (b) MWCNT/AuNP decorated electrode

4.4 TEM images of (a) MWCNT, (b) MWCNT/AuNP and (c) MWCNT/AuNP/XO decorated electrodes

4.5 AFM images of MWCNT/AuNP nanocomposite electrodes at different depths of (a) $164.5 \mathrm{~nm}$, (b) $173 \mathrm{~nm}$ and (c) $1.6 \mu \mathrm{m}$....

4.6 Nyquist plot showing variation of impedance on electrodes functionalized with MWCNT and MWCNT/AuNP at $5 \mathrm{mV}$ between $1 \mathrm{mHz}$ and $100 \mathrm{kHz}$ in presence of buffer

4.7 Schematic showing electron transfer mechanism in xanthine oxidation by XO and HRP on a nanocomposite functionalized electrode

4.8 (a) Comparison of $\mathrm{CV}$ signals of various $\mathrm{XO}$ electrodes in presence of xanthine, (b) CV signals of various xanthine concentrations from MWCNT/AuNP/XO biosensor. Inset: Linear regression plot of the biosensor

4.9 (a) CV signals of bare, MWCNT/Au functionalized with XO or HRP or both in presence of $32 \mu \mathrm{M}$ xanthine, (b) CV signals of bare, $\mathrm{XO} / \mathrm{HRP}$ electrodes with MWCNT or Au or MWCNT/Au in presence of $32 \mu \mathrm{M}$ xanthine; Insets: (c) Linear regression plot of the bi-enzymatic sensor, (d) Linear regression plot of the sensor at elevated concentrations 
5.1 (a) Schematic displaying the working mechanism of Corona Plasma in air,

(b) Experimental setup of electrode treatment using Corona Plasma

5.2 Schematic displaying diffusion of analyte from sweat glands through underlying dermal and epidermal layers of skin

5.3 Survey form handed out to the volunteers from whom human sweat samples were collected

5.4 Steps involved in sweat sample collection

5.5 De-identified wound dressings from 5 patients, subjects 1-5 respectively are shown by green dashed lines (---); red dashed lines (---) represent the extraction area of wound exudate; blue dashed lines (---) represent the extraction area of biofluid from perilesional skin and pink dashed lines (---) represent healthy skin extracted area ...85

5.6 (a) Steps involved in IOT electrode preparation, (b) Screen printing using conductive graphite paste, (c) Precise cutting of electrode design, (d) Integration of flexible electrode on dressing, (e) Flexible electrode on wound dressing .... 86

5.7 Contact angle measurements recorded from (a) Non-plasma treated and (b) Plasma treated electrodes

5.8 Plot depicting (a) CV response recorded from plasma treated and non-plasma treated electrodes in presence of UA, (b) Increase in response to different increasing concentrations of UA, inset: linear regression plot to increasing UA between $6 \mathrm{nM}$ and $0.4 \mathrm{mM}$

5.9 Effect of low sample volume on (a) $E \mathrm{p}_{\mathrm{a}}$ and (b) $I \mathrm{p}_{\mathrm{a}}$ of the biosensor, where $48 \mu \mathrm{M}$ uric acid was measured. The dotted lines in both graphs represent the upper and lower limits. The plots in the (a) and (b) insets are normal distribution curves representing potential and current, respectively. The inset schematic in (b) is the experimental setup

5.10 Sample plot depicting CV response of the UOx electrode to 1 of the sweat and wound samples measured at $20 \mathrm{mV} \mathrm{s}^{-1}$. This data shows wound has higher concentration of uric acid than sweat as reported

5.11 Plot representing the effect of fat emulsification in sweat on the biosensor recovery data obtained through DPV technique. S1, S2 and S3 represent 30.3, 33.9 and $37.5 \mu \mathrm{M}$ uric acid respectively. The top and bottom vial images are the clear and fat emulsified sweat samples

5.12 De-identified wound images of patients diagnosed with venous leg ulcers ( $a, b)$ and sickle cell disease leg ulcer (c) 
5.13 Concentrations of xanthine concentrations from extracts of biofluids sampled from (a) healthy skin, (b) perilesional skin and (c) wound exudate of five de-identified patients using the biosensor

5.14 Plot depicting percentage change in current response of the biosensor as a function of volume of extracted biofluids from (a) healthy skin, (b) perilesional skin and (c) wound exudate

5.15 (a) Flexible electronics; (b) Schematic of the potentiostat and connections;

(c) Circuit design of components embedded on the PCB interfaced with the flexible wound sensor

5.16 Bland-Altman plots representing variation in (a) current and (b) potential from studies using classical setup and IoT platform in presence of $100 \mu \mathrm{M}$ uric acid, measured at $20 \mathrm{mV} \mathrm{s}^{-1}$

6.1 Schematic representing the convergence of smart wound care management and IOT 


\section{LIST OF ACRONYMS AND ABBREVIATIONS}

$\begin{array}{ll}\text { POC } & \text { Point of care } \\ \text { UA } & \text { Uric Acid } \\ \text { IOT } & \text { Internet of Things } \\ \text { PDMS } & \text { Polydimethylsiloxane } \\ \text { ATP } & \text { Adenosine triphosphate } \\ \text { XOR } & \text { Xanthine oxidoreductase } \\ \text { XO } & \text { Xanthine oxidase } \\ \text { ROS } & \text { Reactive oxygen species } \\ \text { TMB } & \text { Tetramethylbenzidine } \\ \text { TRIAP } & \text { 1,1,3-tricyano-2-amino-1-propene } \\ \text { FTIR } & \text { Fourier transform infrared } \\ \text { FI } & \text { Flow injection } \\ \text { HPLC } & \text { High-performance liquid chromatography } \\ \text { GCMS } & \text { Gas chromatography/mass spectrometry } \\ \text { DOP } & \text { Dopamine } \\ \text { AA } & \text { Ascorbic acid } \\ \text { UOx } & \text { Uricase } \\ \text { ITO } & \text { Indium tin oxide } \\ \text { GO } & \text { Graphene oxide } \\ \text { SWCNT } & \text { Single-walled carbon nanotubes } \\ \text { DWCNT } & \text { Double-walled carbon nanotubes } \\ \text { MWCNT } & \text { Multi-walled carbon nanotubes } \\ \text { PC } & \text { Polycarbonate } \\ \text { PET } & \text { Polyethylene } \\ \text { PI } & \text { Polyimide } \\ \text { PEDOT:PSS } & \text { poly (3,4-ethyl-enedioxythiophene)-poly(styrene-sulfonate) } \\ \text { PVDF } & \text { Polyvinylidene fluoride } \\ \text { PTFE } & \text { Polytetrafluoroethylene } \\ & \end{array}$




$\begin{array}{ll}\text { PANI } & \text { Polyaniline } \\ \text { PMMA } & \text { Polymethyl methacrylate } \\ \text { VOC } & \text { Volatile organic compound } \\ \text { PVA-SbQ } & \begin{array}{l}\text { Poly (vinyl alcohol) N-methyl-4(4'formylstyryl) pyridinium } \\ \text { methosulfate acetal }\end{array} \\ \text { FCA } & \text { Ferrocene carboxylic acid } \\ \text { SPCE } & \text { Screen printed carbon electrode } \\ \text { PBS } & \text { Phosphate buffer solution } \\ \text { HRP } & \text { Horseradish peroxidase } \\ \text { CV } & \text { Cyclic voltammetry } \\ \text { DPV } & \text { Differential pulse voltammetry } \\ \text { SEM } & \text { Scanning electron microscope } \\ \text { EIS } & \text { Electrochemical impedance spectroscopy } \\ \text { DET } & \text { Direct electron transfer } \\ \text { MET } & \text { Mediated electron transfer } \\ \text { FAD } & \text { Flavin adenine dinucleotide } \\ \text { DMF } & \text { Dimethylformamide } \\ \text { AFE } & \text { Analog front end }\end{array}$




\section{CHAPTER 1}

\section{INTRODUCTION}

\subsection{Motivation}

A growing need exists for personalized medicine in global healthcare. Smart healthcare technologies draw the attention of scientists and researchers to bring breakthroughs in medical science. Accordingly, there is a demand for robust, flexible biosensing platforms in clinical applications for disease diagnosis and treatment monitoring.[1] Provision of point-of-care (POC) medical facilities can offer the tracking of vital health parameters and, in turn, reduce the outcomes of delayed treatment and associated costs. Wearable biosensing has of late garnered considerable interest in personal diagnostics and health management. There is renewed interest in flexible platform technologies due to their low cost, light weight, low modulus and high stretchability.[2] Such intrinsic factors are paramount to the convenient provision of health parameters on a flexible, biocompatible platform that can facilitate timely decisions for effective therapy. These platform technologies aim to provide alternative solutions to POC diagnostic assays for broad applications in personalized medicine and offer valuable insights into one's physiological conditions, from continuous monitoring of an individual's biomarkers in real-time.

Commercially available biosensors are based on conventional electronic technology that is yet to adhere perfectly to wearable-form factors and conditions. Quantum advancements are being made to engineer soft, flexible biosensors with tunable electrochemical and electromechanical properties. These are expected to provide accurate and reliable sensing without compromising on the natural movement and 
comfort of its users.[3] Although wearable biosensing gains significant attention in personal health management, there are constraints interfering with reliable and convenient operation from a wearable construct. Such constraints can be attributed to differences in electrode-skin interface impedance and contact surface area, that affect the reproducibility and stability of performance. The incompatibility between existing techniques and flexible substrates, demands a revolution in material science and the fabrication processes involved.[4]

Detection of physiological biochemical indicators can utilize the natural human metabolic pathways to assess wellness parameters. This process entailing the use of enzymes provides for a natural, biocompatible approach in biosensing. Inclusion of enzymes in detection is influenced by the catalytic properties of the enzyme activity and its stability over time. Enzymes, which act as catalysts in a bio-chemical reaction, are reportedly active at specific $\mathrm{pH}$ and temperature, and are known to fade over variations. [5] As a result, stability of enzymatic response at higher temperatures and different $\mathrm{pH}$ can vary over time.

Pertinent factors that affect the electrochemical response of a system arise from variations in electrode distances and area, which can consequentially alter performance. This work focuses on addressing some challenges faced in electrochemical detection of specific biomolecules from a wearable construct. Possible variations in response stability have been addressed through precise selection of substrate materials and robust enzyme immobilization protocols. Surface modification methods have been utilized to improve substrate hydrophilicity and enable higher enzyme loading for improved response and minimal variation in stability. Nano-material scaffolds have been 
employed for improved surface interactions and accelerated electron transfer. The integration of specific nano-composite material enables enhanced response with minimal variation.

Electrochemical analyses have been conducted using enzymatic sensors designed on different substrate materials, to assess any degrade in sensor stability and reproducibility under physiologically relevant variations. Enzymatic sensors can be designed at a cost-effective, miniaturized scale through micro/nano fabrication processes. The use of enzymes enable quick detection with low response time and can potentially offer the tremendous advantage of convenient, personalized therapy. [6]

Conventional wound-care protocols are primarily visual or entail colorimetric and optical detection approaches to assess lesion width and depth. These systems are bulky, expensive, and do not provide rapid results for a comprehensive understanding of tissue recovery. In this study, purine metabolites such as uric acid (UA) and Xanthine, which can report changes in tissue recovery from longitudinal measurements, have been investigated as target biomarkers for wound monitoring, through enzymatic detection. Sensor material and design configurations have been carefully tailored, and specific techniques have been employed to achieve minimal variation in response within the concerned physiological ranges. The need for this study has thus been, to design electrochemical sensors on a wearable construct, and to identify and engineer stable pathways for enzymatic detection of the concerned biomarkers in wound monitoring. 


\subsection{Specific Aims and Objectives}

The specific objectives in this study have been as follows:

i. Examine the effects of enzymatic performance for minimal variation under relevant conditions of temperature and $\mathrm{pH}$

Hypothesis - Enzymes fade over variations of $\mathrm{pH}$ and temperature, that affects sensor response.

ii. Investigate a nanocomposite of MWCNT/AuNP for enhanced sensing response Hypothesis - Composite of MWCNT/AuNP nano-scaffolds increase electroactive area, surface conductivity and binding sites, thereby increasing sensor signal, as compared to their isolated presence or absence on bare or enzymatic electrodes.

iii. Understand the effects of sensor material properties on substrate functionalization for improved performance

Hypothesis - Increased hydrophobicity of sensor surface reduces loading and functionalization across the substrate surface, thereby attenuating sensor response.

iv. Validate sensor performance through secondary analysis of extracted clinical samples of biological fluids against standard colorimetric assay.

A nano-composite functionalized enzymatic sensor was designed in this study for the investigation of target biomolecules in extracts of wound exudate and sweat from around perilesional and healthy skin. Correlations obtained provide for a non-invasive method to quantify wound severity and healing progress from around wound milieu.

\subsection{Dissertation Organization}

In Chapter 2, a comprehensive discussion on existing state-of-the-art wearable biosensing and wound care detection is presented, with a review of the advantages and 
limitations of conventional techniques. The physiology and relevance of wound care biomarkers, namely UA and Xanthine, are discussed herein, elucidating their biochemical correlation with wound monitoring. Besides these bio-physiological indicators, certain environmental indicators $(\mathrm{pH}$, temperature) are also discussed. An overview of the different kinds of materials and techniques being explored in such detection have been reported.

Chapter 3 presents the details of experimental procedures employed in enzymatic detection of UA. Suitable substrate functionalization and enzyme immobilization techniques that were adopted in investigating UA detection, have been stated. Characterization methods to explore the stability of enzymatic detection have been elaborated in this chapter. The feasibility of detection under physiologically relevant environmental variations is reported herein.

Chapter 4 presents the details of experimental protocols utilized in Xanthine detection. Synthesis of a hybrid nanocomposite matrix which assisted in maintaining enzyme stability to obtain enhanced signal, has been described here. Imaging analyses of the nanomaterial modified sensor surface have offered information on surface morphology, roughness and conductivity. Analysis of the electrochemical response obtained from the nanomaterial functionalized electroactive substrates have been evaluated in this chapter.

Chapter 5 presents the effects of sensor material properties on performance. Specific techniques utilized to improve hydrophilicity of the sensor surface to achieve increased functionalization and loading for higher signal, have been reported here. Secondary analyses from clinical extracts of physiologically relevant biological fluids have been investigated to validate sensor response. Feasibility for potential non-invasive detection of 
target bio-analytes has been considered here. The viability of sensor design and detection from a flexible, healing platform has been assessed in this chapter for wearable wound monitoring.

Chapter 6 summarizes the design, fabrication, and characterization of electrochemical sensors for wearable wound monitoring applications. Concluding remarks and prospects of the research work undertaken have been directed in this chapter. 


\section{CHAPTER 2}

\section{LITERATURE REVIEW}

\subsection{Wearable biosensing}

A wearable biosensor is a digital device that can be worn on the body in combination with a self-contained integrated device capable of providing specific quantitative analytical information. It does so by using a biological recognition element which is in direct spatial contact with a physiochemical transducer. An electrochemical wearable biosensor is a body-borne system that is intended to continuously interact with the wearer and produce an electronic signal proportional to a single bio-analyte.[7] The acquired bio-physiological information can then be conveyed to an associated electronic device and sent wirelessly to healthcare providers. This two-way feedback between a patient and doctor made possible at the remote level can offer substantial potential in medical IoT (internet of things), allowing to bring medical assistance to a personalized scale.[8]

Wearable biosensors can be made operational based on three important criteria: wearability, reliability and convenience. Without wearability, utility of the device will be limited to users who demand valid data for informed clinical decisions. For the system to be wearable, the users' convenience and comfort are of upmost importance, without which user engagement will not be achieved.[9]

\subsubsection{Current state-of-the-art in wearable biosensing}

Device geometries for precise body to signal transduction include systems with largescale set-ups designed for laboratory settings. This includes primitive functional platforms that integrate consumer electronic goods, such as wrist-mounted wearables, to newly emerging skin-like devices that combine the attractive features of the two. 
A lot of research has been continuing in body-sensor-networks to integrate biosensors into wearable platforms like watch, bracelet, belt, patch, eyewear and textile; made functional with the use of smart technology. Such systems are of late gaining immense interest for providing continuous measurements of various biometric data. Currently, stateof-the-art devices in wearable biosensing typically include measurements of different physiological parameters like heart rate, blood pressure, wake-sleep patterns, respiration rate, oxygen levels, blood glucose levels and physical activity in real time. [10,11]

In comparison, relatively little has been accomplished in wearable biochemical sensors, which can be useful for personal management of wellness, through non-invasive monitoring of target biomarkers. This is possible through the detection of relevant biomolecules from different biological fluids such as sweat, saliva, tear and urine of the

user. The accomplishment of such sensors has been a mixture of both progress and setbacks.[12] Most commercial advancement is being made with the smart adaptation of existing mechanical, electrical and optical techniques of measuring concerned parameters. This involves innovation in miniaturization of sensing technologies to make them conformal to the skin surface.[12] Challenges associated with specific detection of concerned analytes, attribute to interference from other predominant analytes in the sampling matrix, and the impedance associated with diffusion of ions through different layers of the skin. [13-14]

\subsubsection{Detection mechanisms explored in wearable biosensing}

Capacitive pressure sensors are being employed in human pressure sensing interfaces to mimic tactile sensation and joint bending detection. For example, the flexible parallel plate capacitive system by Pressure Profile Systems, Inc. (PPS) (Figure 2.1a) has the ability 
of flexible tactile sensation and body pressure mapping.[15] By results of ease of improvements in design flexibility and stretchability, new electrode materials for capacitive sensors thus draw awareness.[16] To best match skin modulus, silicone elastomers like polydimethylsiloxane (PDMS) are being used to design such skin conformal biosensing systems. Detection of human radial artery pulse waves is being explored using capacitive pressure and strain sensing using micro-patterned conductive polymer films. Piezo-resistive effect of strain sensors are known to be used in wearable electronics to detect human physiological movement.[17] For reconstructing wrist and finger tactile sensations, ionic liquids are being employed as sensing elements to resolve contact forces in flexible and transparent microfluidic systems.[18]
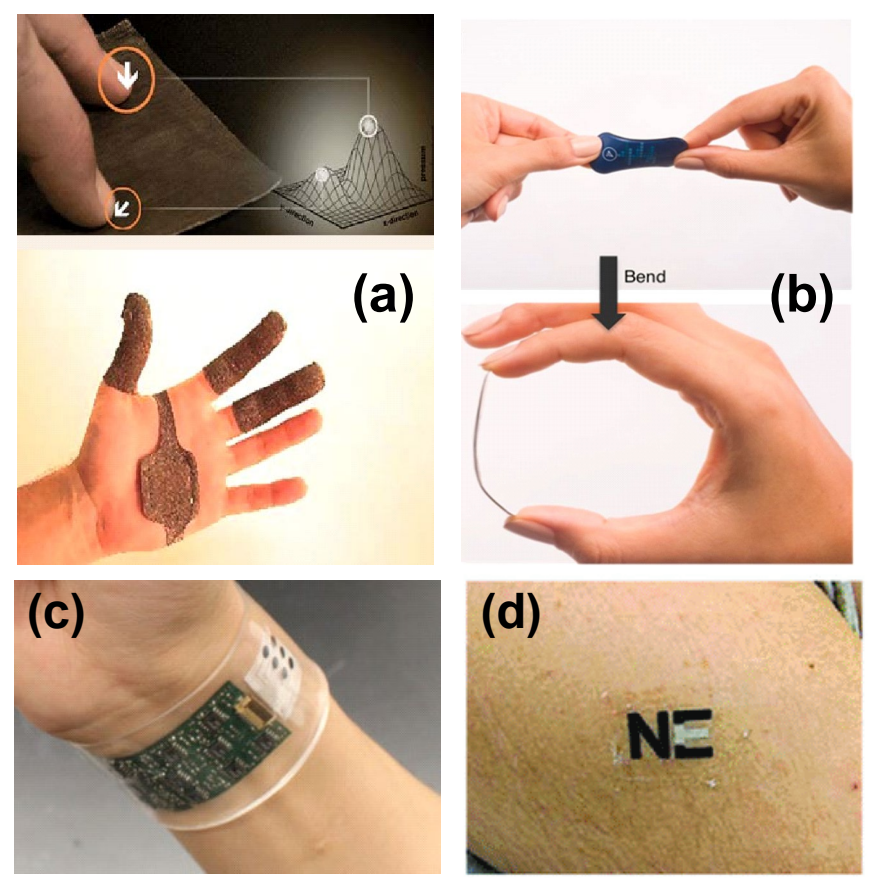

Figure 2.1. (a) TactArray, flexible capacitive array tactile sensor from Pressure Profile Systems[15], (b) Flexible, wearable disposable cardiac biosensor for remote and ambulatory monitoring[19]; (c) Wearable sensor array for multiplexed in situ perspiration analysis[21]; (d) Tattoo biosensor for monitoring electrolytes from sweat[26] 
Electrophysiological sensing techniques provide measurements of ECG, EKG, EMG, EOG, and EEG. Besides tracking body motion and temperature using thermal sensing, optical detection is employed by compact wearable diagnostic devices to capture heart rate and oxygenation (Figure 2.1b).[19] In order to acquire the required biological information, the processes of light scattering and absorption on skin are employed. Although qualitative, chemical-to-optical transduction, enable cost-effective detection with system design simplicity. Such methodologies establish clinical relevance in the assessment of cardiovascular, myocardial and tissue health.[12]

Electrochemical sensing for continuous monitoring of chemical biomarkers at the molecular level in real-time is yet to be achieved in wearable biosensing. Such sensing systems operate through chemical-to-electrical signal conduction, generating an electrical signal from a chemical reaction between ions in a sampling biomatrix and a transducer.[20] This can be attained from direct access to analytes in bio-fluid matrices secreted from the body.

These systems can meet the criteria for wearable biosensing owing to their inherent miniaturization ability, low power requirements, simplicity, speed and low-cost fabrication. Recent efforts have demonstrated the design of epidermal electrochemical sensors that can endure mechanical strains and stretching on skin conformal surface with minimal compromise on performance. Multi-analyte electrochemical sensing platforms are being designed on wristband based wearable constructs, to monitor the wearer's body temperature and levels of glucose, lactate, potassium, sodium from human sweat. Body compliant spatio-temporal mapping of localized ion concentration has been achieved to monitor transient electrolyte concentration profiles and then alert the wearer of potential 
health risks. This multi-sensor epidermal platform (Figure 2.1c) merges plastic-based sensor arrays with silicon integrated circuits consolidated on a flexible circuit board for advanced signal conditioning, processing and transmission.[21] Such sensors utilize enzymes pertaining to human bio-chemical pathways as natural catalysts to enhance sensing performance. This shows the biocompatibility of enzymes, with excellent specificity to the concerned biochemical pathway.

Among different sampling biomatrices being explored for minimally invasive detection of bioanalytes; sweat, saliva and tear are known for the presence of multiple physiologically relevant chemical constituents. Flexible tattoo and textile based amperometric and potentiometric sensors as seen in Figure 2.1d demonstrate the detection of certain analytes like glucose[22-25] and lactate[26-29] from human sweat, along with determining the presence of ions such as calcium[30], ammonium[31] and sodium[28, 3234].

\subsection{Biochemistry of wounds}

Purine metabolism (Figure 2.2) is known to play an integral role in tissue regeneration and wound healing. Physiological wound healing requires energy in the form of ATP (adenosine triphosphate).[35-36] With the degradation of purines in purine metabolism, each step in the cycle generates a considerable amount of energy. This is known to grow with increased apoptosis, leading to increased production of products in the pathway. In turn, the terminal catabolite of purine metabolism in humans, uric acid, rises. This analyte has been closely linked with relatively increased concentrations in the case of an injury, when cells are increasingly dying.[37] Disturbance of the normal healing process of a 
wound, leads to sustained increased expressions of pro-inflammatory cytokines, linked with increased UA.[38]

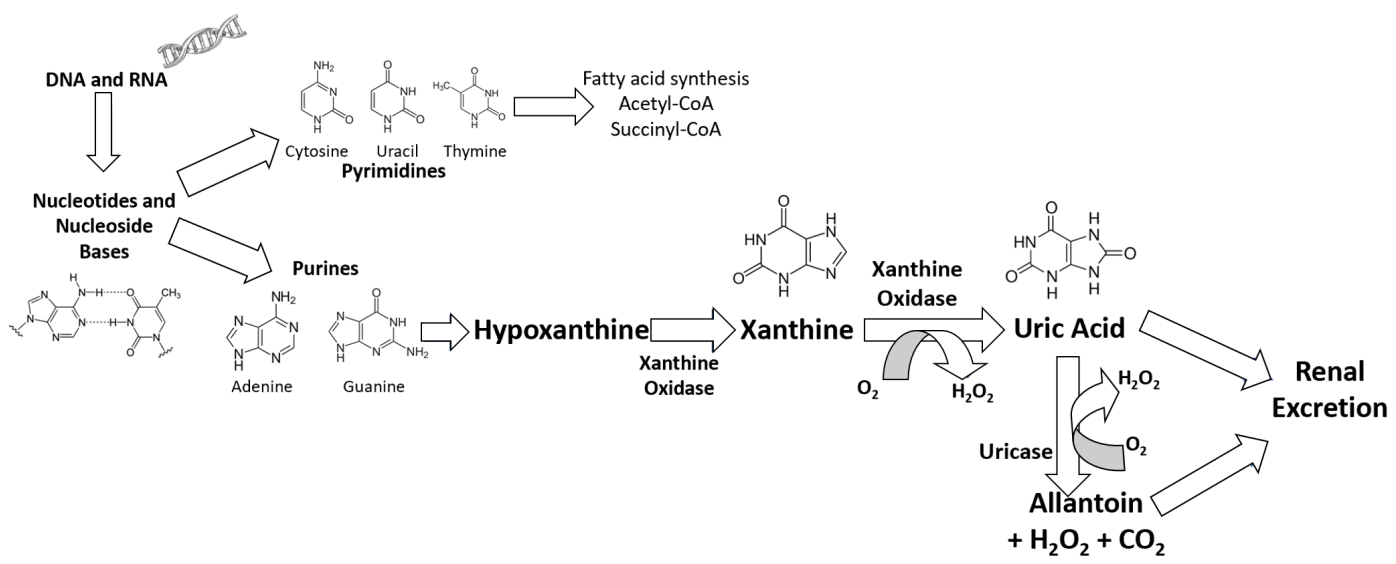

Figure 2.2 Purine metabolism in humans from apoptosis to excretion of Uric Acid

\subsubsection{Biomarkers in wound care}

\subsubsection{Uric Acid}

Uric Acid is the terminal product of metabolic breakdown of purine nucleotides, Adenine and Guanine (Figure 2.2). It is a weak organic acid that, under normal physiological conditions, exists mainly as a monosodium salt.[39] It is continually produced by cells in the human body and is eventually excreted by the kidneys as a normal component of urine. Known to form ions and salts called urates,[39] UA is a heterocyclic compound of carbon, nitrogen, oxygen and hydrogen bearing the chemical formula, $\mathrm{C}_{5} \mathrm{H}_{4} \mathrm{~N}_{4} \mathrm{O}_{3}$ as illustrated by the distribution of atoms in Figure 2.3a.

The normal blood concentration of UA in humans varies between 0.1 and $0.4 \mathrm{mM}$ $\mathrm{L}^{-1}$. Elevated levels of UA have been correlated with its accumulation in kidneys as urate stones and as crystals in joints, leading to gout. Increased concentrations of UA also lead to hyperuricemia, joint inflammation, ischemia and increased risks of Type 2 
diabetes.[40y] Relatively higher UA concentrations are associated with $40 \%$ increased diabetic risks and $70 \%$ increased renal risks. A specific consequence of diabetes is
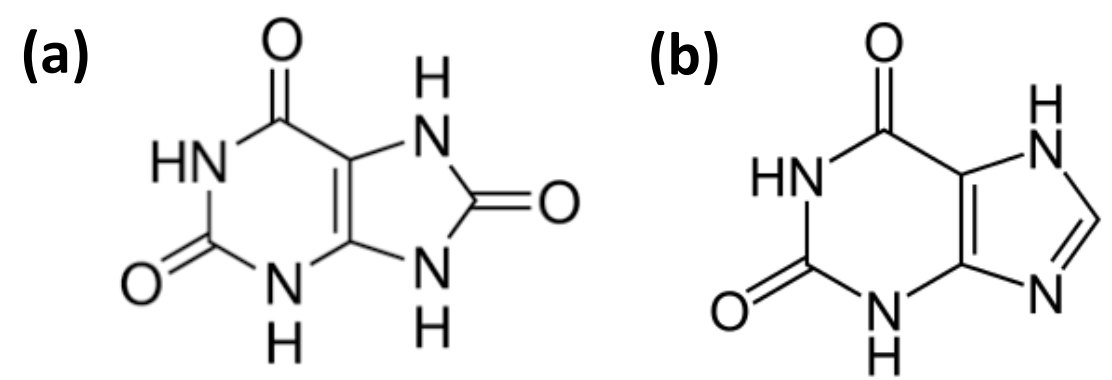

Figure 2.3: Chemical structure of biomolecules (a) Uric Acid (b) Xanthine

neuropathy, which refers to the inability of neurons to send impulses. This reportedly affects almost $60-70 \%$ of diabetic patients.[41] This makes the timely assessment of wound occurrence and its management a challenge. Many diabetics are thus inflicted with chronic wounds, which bear huge socio-economic impacts in treatment management, along with associated trauma from consequences of surgeries and amputations.[42]

Increased levels of UA, the product of human purine metabolism[43], is connected with increased breakdown of nucleotides and cell death. This indicates its role as a possible biomarker in wound care. Correlations between UA in the wound fluid and chronic venous leg ulcers has been established. [44, 45] Chronic, non-healing wounds reportedly contain elevated levels of UA, which lower with time as the wounds heal (Figure 2.4). Thus, possible correlations of UA concentrations can be drawn from the healing of a wound through its phases. POC techniques, which involve the detection of UA, can enable quantitative assessment of a wound's recovery. 


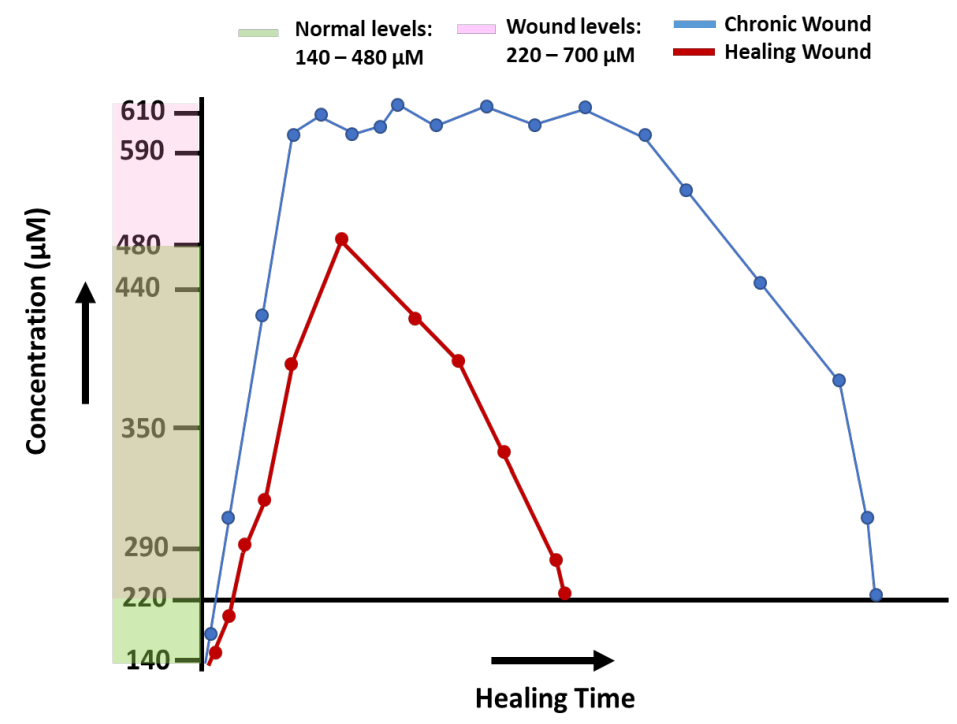

Figure 2.4: Correlation of Uric Acid concentrations with wound healing [243]

On the occurrence of a wound, UA levels are known to increase to about $0.2-0.7$ $\mathrm{mM} \mathrm{L}^{-1}$ from its normal concentration. With elevated concentrations around an injury, this analyte can act as a potential biomarker to monitor healing progress from an injured micro-environment. After phase one (hemostasis), when initial bleeding is arrested to prevent further clots, new blood vessels start forming with tissue regeneration and epithelialization of the extracellular matrix in second phase of inflammation. This is followed by increased tensile strength of the matrix in phase three of proliferation with re-modelling toward complete recovery from freshly healed dermal and epidermal skin layers. Severity of a wound can be determined by the number of days a wound takes to heal through its multiple overlapping phases, with increased chronicity being depicted from higher concentrations of UA in phases of inflammation and proliferation. For normal healing of wounds, the levels tend to gradually reduce, proceeding towards the final phase of remodeling and complete recovery.[46] Across the different healing phases 
of a wound, UA levels are known to be higher in phases two (inflammation) and three (proliferation). A chronic wound is known to have a slower healing rate over prolonged inflammation with increased infection.

Besides blood, plasma $(\sim 0.3 \mathrm{mM})$, serum $(0.2 \mathrm{mM}-0.4 \mathrm{mM})$ and urine $(250-750$ $\mathrm{mg}$ /day), UA is also found in biofluids of sweat $(\sim 24.5 \mu \mathrm{M})$, saliva $(0.1 \mathrm{mM}-1 \mathrm{mM})$ and tears $(0.07 \mathrm{mM}-0.1 \mathrm{mM})$, although in lower concentrations.[47-53] This allows for possible non-invasive detection of UA. Different detection procedures of UA have been investigated, but that for personalized wound monitoring, is still relatively in its infancy. Primitive studies have suggested that detection of UA from wound exudate holds potential for continuous monitoring of healing. Existing protocols explored for UA detection involve optical methods of colorimetry or fluorometry, where either a fluorometric dye is used or the differential absorbance of the reactant and product of its enzymatic oxidation are noted at a certain wavelength $(293 \mathrm{~nm})$. Other approaches in detection involve chemical,

fluorimetry, spectrophotometry, chemiluminescence, high performance liquid chromatography and isotope dilution mass spectrometry.[54-58]

\subsubsection{Xanthine}

Xanthine is referred to as the cell housekeeper with major roles in cellular metabolism through maintenance of cell redox potential, bacterial scavenging, infection inflammation, antioxidant defense and system detoxification. Created from hypoxanthine by xanthine oxidoreductase (XOR), xanthine $\left(\boldsymbol{C}_{5} \boldsymbol{H}_{4} \boldsymbol{N}_{4} \boldsymbol{O}_{2}\right)$ (Figure 2.3b) is an intermediate product of purine degradation. Its oxidation to UA is catalyzed by enzyme xanthine oxidase (XO), a form of XOR which generates reactive oxygen species (ROS). 
In the case of cell rupture, xanthine is formed in the wound (localized xanthine) from the breakdown of released energy of metabolites in damaged tissue. Inflammation is a necessary response to tissue injury, leading to the production of ROS to mediate oxidative stress, critical to normal wound healing.[60] Such species act as scavengers of oxygen radicals to facilitate tissue recovery. Generated ROS from xanthine oxidation, can thus alleviate healing and tissue repair.[59] Increased apoptosis increases XO activity thereby raising subsequent metabolite levels. This suggests that wounds can thus be monitored until closure through assessments of xanthine levels, to obtain an improved understanding on healing.[61]

This bio-analyte is a purine base found in different human bio-fluids. Besides blood $\left(65-130 \mu \mathrm{M} \mathrm{L}^{-1}\right)$, xanthine is also present in lower concentrations in other biological fluids like plasma $(1.4 \mu \mathrm{M})[62]$, serum $(4.3-10.3 \mu \mathrm{M})[63]$, urine $(\sim 3.9 \mu \mathrm{M})[64]$ and tears[65]. This shows that potential non-invasive detection of xanthine is possible. Detection of xanthine has been explored using different optical based methods like colorimetric and fluorometric approaches whereby differential absorption of the analyte's oxidation reactant/product is analyzed at specific wavelength $(570 \mathrm{~nm})$. Mass spectrometric and chromatographic techniques (HPLC) have also been explored to determine concentrations of the analyte from different biofluid samples. $[66,67]$ However, techniques for detection from biofluids associated with a wound milieu, are still on an anvil.

\subsection{Monitoring the wound environment}

Research into a wound milieu has shown that ambient parameters namely $\mathrm{pH}$, temperature and moisture from injured tissue environment, can offer indications of healing. Some conventional real-time wound monitoring biosensors are reported in Table 2.1. 
All human biochemical processes are influenced by $\mathrm{pH}$ balance. Therefore, with $\mathrm{pH}$ as an established health indicator, it can be correlated to cellular, protease activity and

\begin{tabular}{|c|c|c|c|c|c|}
\hline Sensor type & Range & Advantages & Limitations & Approach & Ref. \\
\hline $\mathrm{pH}$ & 2 to 13 & $\begin{array}{l}\text { Assesses pH } \\
\text { shift in } \\
\text { chronic } \\
\text { wounds (up to } \\
\text { pH 12) and } \\
\text { skin pH (4-6) }\end{array}$ & $\begin{array}{l}\text { Low accuracy, } \\
\text { not wearable }\end{array}$ & $\begin{array}{c}\text { Optical, } \\
\text { potentiometric }\end{array}$ & [2-5] \\
\hline Moisture & $1-5$ scale & $\begin{array}{l}\text { Measures } \\
\text { wound } \\
\text { moisture } \\
\text { indicating } \\
\text { dressing } \\
\text { change } \\
\text { requirements }\end{array}$ & $\begin{array}{c}\text { Doesn't give } \\
\text { direct measure of } \\
\text { healing }\end{array}$ & Impedance & {$[5,6]$} \\
\hline Temperature & $22-80^{\circ} \mathrm{C}$ & $\begin{array}{l}\text { Determine } \\
\text { severity from } \\
\text { elevated } \\
\text { temperature, } \\
\text { repeatable, } \\
\text { sensitive to } \\
0.2^{\circ} \mathrm{C} \text { change } \\
\end{array}$ & $\begin{array}{l}\text { Interference with } \\
\text { body } \\
\text { temperature, not } \\
\text { direct } \\
\text { assessment of } \\
\text { healing }\end{array}$ & Resistive & [3] \\
\hline Oxygen & $\begin{array}{c}0-100 \\
\text { mm Hg }\end{array}$ & $\begin{array}{c}\text { Correlation of } \\
\text { tissue } \\
\text { oxygenation } \\
\text { with healing }\end{array}$ & $\begin{array}{c}\text { Implantable, } \\
\text { doesn't provide } \\
\text { direct } \\
\text { assessment of } \\
\text { chronicity, } \\
\text { doesn't provide } \\
\text { continuous } \\
\text { measures }\end{array}$ & Optical & [7] \\
\hline $\begin{array}{l}\text { Microbial } \\
\text { infection }\end{array}$ & - & $\begin{array}{c}\text { Tracks } \\
\text { bacterial } \\
\text { infection for } \\
\text { appropriate } \\
\text { drug therapy }\end{array}$ & $\begin{array}{l}\text { Doesn't provide } \\
\text { direct measure of } \\
\text { healing }\end{array}$ & Optical & {$[3,4]$} \\
\hline
\end{tabular}

Table 2.1. Comparison of conventional real time wound-care biosensors.[236]

bacterial infection. Monitoring the $\mathrm{pH}$ of a wound environment, can assist in evaluating the underlying biochemical processes involved in tissue healing.[68-69] 
Wound occurrence shifts the $\mathrm{pH}$ of injured tissue region from normal, acidic levels of healthy skin (4-6), to alkaline (7-9). As healing progresses, this $\mathrm{pH}$ is known to shift back to acidic. Optical detection protocols have been investigated to monitor the $\mathrm{pH}$ of postsurgical areas. Electrical measurements have been performed using conductive thread based diagnostic device platforms, to demonstrate subcutaneous $\mathrm{pH}$ shift of a wound environment from physiochemical tissue properties. [70] A novel smart bandage designed by Empa Inc. reportedly alerts nursing staff on adverse healing through optical detection (Figure 2.5d). Optical sensors incorporated into a bandage demonstrate qualitative tracking of healing, from $\mathrm{pH}$ variations of injured tissue area, utilizing fluorometric techniques to glow at different intensities based on wound severity.[71] This does not involve unnecessary dressing change to monitor healing, leaving it undisturbed from orientation and position. This gentle therapy of fluorescence sensing intends to provide continuous wound care to patients from home, without having to change dressings more frequently than necessary. However, this does not provide quantitative measures of wound healing progress.

Temperature is a factor known to be associated with inflammation. On the commencement of a physical injury, the temperature in wound milieu can reportedly increase from normal body temperature by $>2{ }^{\circ} \mathrm{C} .[72,73]$ This increased temperature is an indicator of increased infection and inflammation.[69] The difference in temperature from normal, disturbs optimal metabolite functionality in the body, thus creating differences in the involved biochemical and metabolic pathways. This can delay and slow down healing progress.[74] As a wound heals, normal physiological temperature is 
gradually attained, promoting optimal functioning of bio-analytes, contributing to tissue recovery.

Optimal moisture balance is essential for tissue healing, as too wet of an environment can lead to maceration, while too little can desiccate it.[75] Attention has been given to the moisture condition of a wound milieu at the time of dressing change. Commercial WoundSense moisture sensor has been reported to observe the wound moisture status, without disturbing or removing the dressing (Figure 2.5e).[76] Studies from different kinds of chronic wounds (diabetic foot ulcers and pressure ulcers), have shown that this system can alert a patient for a dressing change based on optimal moisture levels of the wound environment.[76] This device targets to improve wound management from impedance responses of the sensor, based on shifts in moisture content of the wound.

Tracking bacterial and microbial infection is also considered for appropriate drug therapy, using optical approaches.[77-78] Although these methods claim to determine oxygen measures from a laceration through correlations of optimal tissue oxygenation, these are not directly reflective of recovery status. These correlations are unsuitable for continuous monitoring of tissue recuperation and being incapable of providing direct quantitative measures of healing, cannot be considered for precise evaluation of wound chronicity. A medical breakthrough, that can quantify the healing of injured tissue from longitudinal measurements of specific bio-analytes and enable continuous tracking of wound healing progress, is yet to come. Detection of specific biomarkers of healing, can assist in overcoming some existing limitations in wound care through direct provision of quantifiable measurements from relevant analyte concentrations. This can potentially enable to improve the efficacy of wound management. 


\subsection{Current state-of-the-art in wound care}

Current wound therapy involves frequent clinical trips for treatment management. Assessment of healing is only achieved at the time of dressing change through visual inspection. Imaging solutions like those from eKare Inc. [79] provide information on healing from 3D measurements of a scanned image collected from the wound area. The wound is optically mapped to a visual interface to obtain its physical dimensional
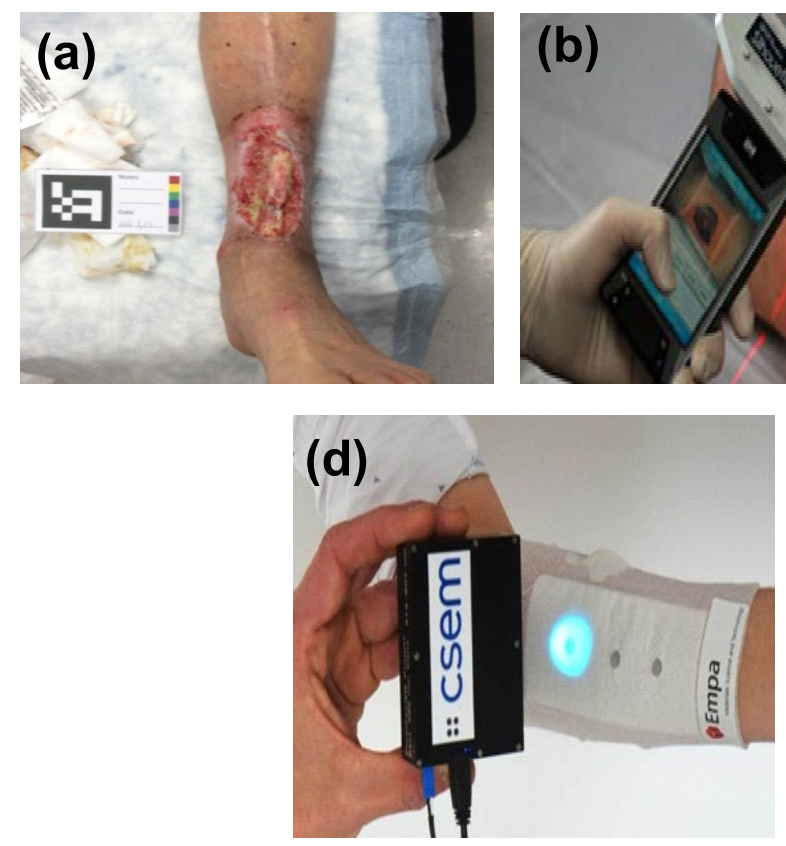
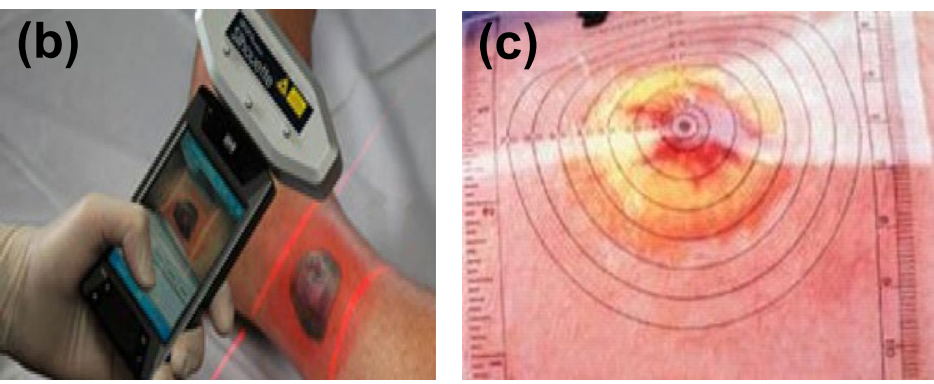

Figure 2.5: Existing protocols in wound management offering qualitative assessment from (a) visual inspection [80], (b) wound imaging[80]; and (c) wound mapping[242]; (d) $\mathrm{pH}$ monitor from Empa Inc. for tracking healing of wounds[71]; (e) Wound moisture monitor from WoundSense[77]

measurements. Aranz Medical Inc. uses laser technology, to scan the wound surface area and monitor healing progress over time.[80] Paper based scales are also available for tracking wound height and width to determine healing through reduction in geometric dimensions and physical parameters. [81- 82] These methods are illustrated in Figure 2.5(ac). However, these conventional practices allow for one-time measurements and offer qualitative assessment. They are not suitable for continuous monitoring and do not offer 
convenient compliance with treatment protocols, with inefficient management of wound dynamics in real-time.

\subsection{Mechanisms explored in detection of wound biomarkers}

An overview of the approaches utilized in detection have been enlisted below:

i. Colorimetry - Colorimetric approaches in optical detection are used for determining purine metabolites like UA and xanthine. Such procedures for assays in serum and urine have been previously investigated. Morphological transition of Ag nano-prisms at a specific wavelength can be used to determine UA concentrations from a color change through a shift in surface plasmon resonance spectral band.[83] Microfluidic paper-based analyses have also shown bi-enzyme colorimetric detection of different concentrations in human serum samples. With enhanced sensitivity, change in color signal has enabled detection from small volumes.[84] Such platforms have provided improved color uniformity and intensity for sensitive and selective determination of UA, from co-immobilization of chromogenic reagents (tetramethylbenzidine, TMB) generating a colored product, also enabling detection at lower limits (1 $\mu \mathrm{M})$.[85] Colorimetric studies from palladium (Pd) nanoparticles supported on nitrogen and sulfur doped nanostructures with TMB have provided efficient detection of Xanthine and offered information form urine concentrations. [86] This method used to evaluate Xanthine in urine and serum samples was seen to provide good stability.[87]

ii. $\quad$ Fluorimetry - Fluorescent sol-gel biosensors have been designed for determining concentrations in urine, serum and blood. With detection limits as low as $20 \mathrm{nM}$, studies have shown reliable determination from diluted biological fluids in presence of interfering species.[88] Approaches have been used to understand the fluorescent 
reaction between UA/Xanthine and a fluorometric reagent in the presence of hydrogen peroxide $\left(\mathrm{H}_{2} \mathrm{O}_{2}\right)$ and TRIAP (1,1,3-tricyano-2-amino-1-propene), which generates a fluorescent product at a specific wavelength, observed from their respective excitation and emission spectra.[89] Fluorometric assays for determining analyte concentrations in serum and urine have been shown using direct reaction rate methods. Consumption of dissolved oxygen at a certain potential has been correlated with the analyte's presence. Fluorometric assays have reportedly demonstrated good sensitivity to Xanthine in tissue.[90]

iii. Spectrophotometry - Catalytic activity of the specific enzymes can be studied from enzyme functionalized substrates using spectrophotometry. Radiation grafting of functional polymers, supported the design of an optical biosensor to sense shifts in UA/Xanthine concentrations, in the micro-molar range $(<200 \mu \mathrm{M})$, in aqueous solutions. This was observed from the SPR band of its UV-Vis absorption spectra in presence of varying concentrations.[91] Spectrophotometric investigations were conducted for detection of purine metabolites in serum samples, depicting a correlation of 0.9 . This study reportedly provides excellent specificity with minimal interference.[92] Such techniques have shown improved sensitivity over other conventional methods. This can be applied to purine metabolite assays for human serum and urine.[93] Enzyme XO activity was monitored in human plasma using a spectrophotometric assay using ferricytochrome c.[94] Fourier transform infrared (FTIR) spectrophotometry has shown the possibility of Xanthine detection using such approaches.[95-96] Multivariate statistical tools have also been used for detection of UA and Xanthine in bio-matrices of human serum and urine. [93] 
iv. Chemiluminescence - Chemiluminescent methods using flow injection (FI) are being developed to determine concentrations of these bio-analytes, based on the enhancement in chemiluminescence intensity of certain compounds in presence of the specific compound. This method was tested with clinical samples of human serum and urine, targeting diagnosis and therapy of patients with disorders such as gout, hyperuricemia and Lesch-Nyhan syndrome, associated with elevated levels of UA.[97] A novel paper based microfluidic platform employing chemiluminescence to detect UA shows a good shelf life, approximately 10 weeks, when stored after single use at $4{ }^{0} \mathrm{C}$. This reports the successful integration of chemiluminescence and a microfluidic paper-based analytical device for an economical, simple-to-use, portable biosensor for low-volume determination.[98] Fiber optic sensors were designed for detection of xanthine based on such methods, from peroxide generated by the reaction of xanthine with luminol and peroxidase. These showed good recovery between 91 and $102 \%$ from analyzed samples.[99]

v. Mass spectrometry - Specific liquid chromatography-tandem mass spectrometry has been investigated for determination and quantification of intracellular and extracellular purine metabolites in urine. Low detection limits of approximately $0.07 \mathrm{mg} \mathrm{dL}^{-1}$ were obtained for UA with a correlation of about 0.9.[100] Techniques of isotope dilution mass spectrometry have been investigated to detect such analytes in serum. The accuracy of obtained results was evaluated with certified values from gas chromatography/mass spectrometry (GC-MS) reference methods.[101] Similar detection methods by liquid chromatography-electrospray tandem mass spectrometry have allowed detection of xanthine from urine samples.[102] XOR activity can also be 
monitored in serum samples using liquid chromatography/triple quadrupole mass spectrometry methods.[103]

vi. High performance liquid chromatography - HPLC technique has been employed to quantify such purine metabolites as pharmacodynamic biomarkers.[104] Detection in human urine has been noted to provide a detection limit of $0.1 \mu \mathrm{g} \mathrm{ml}^{-1}$, with a recovery of approximately $95 \%$.[105] Determination of such analytes in human urine using ion chromatography, have shown detection limits of $0.5 \mu \mathrm{g} \mathrm{L}^{-1}$.[106] Xanthine detection was performed using HPLC to assess feasibility of detection in plasma and erythrocyte samples.[62] Serum sample analyses for multiple purine metabolites showed good recovery (86 - $93 \%)$ using such approaches.[107]

\subsection{Electrochemical determination of wound biomarkers}

Electrochemical biosensing provides for cost-effective detection with greater simplicity and improved sensitivity.[43] This technique can offer excellent electrochemical activity towards detection of UA and Xanthine, with potential application in determining their concentrations in bio-matrices of serum, urine and plasma. Such detection entails investigating the analyte's oxidation/reduction mechanism in their metabolic pathway, whereby the concerned specific enzymes catalyze the undergoing reaction to generate $\mathrm{H}_{2} \mathrm{O}_{2}$ as a by-product. Yield of the generated by-product, $\mathrm{H}_{2} \mathrm{O}_{2}$ can be utilized to quantify UA and Xanthine. [108] Among electrochemical sensing modalities used for UA/Xanthine detection, potentiometric (cyclic voltammetry, linear voltammetry, square wave voltammetry and differential pulse voltammetry) and amperometric/chronoamperometric methods have been explored.[109-118] 
Specific detection for UA and Xanthine is attained from utilization of enzymes UOx and XO, to explore their respective oxidation pathways as shown in Eq. 1 and 2. These enzymes act as natural catalysts enabling detection through a natural biological process.

$$
\begin{gathered}
\text { Xanthine }+\mathrm{H}_{2} \mathrm{O}_{2}+\mathrm{O}_{2} \rightarrow \text { Uric Acid }+\mathrm{H}_{2} \mathrm{O}_{2} \\
\text { Uric Acid }+\mathrm{O}_{2}+\mathrm{H}_{2} \mathrm{O} \rightarrow 5-\text { hydroxyisourate }+\mathrm{H}_{2} \mathrm{O}_{2}
\end{gathered}
$$

Enzymatic detection helps to address some existing challenges associated with elaborate sample collection and preparation procedures, along with prolonged detection time and analyses, associated with existing protocols that are limited to laboratories and deter the successful commencement of effective biosensing. Such approaches can potentially target quantitative monitoring of biomarkers at a miniaturized and costeffective scale. These methods possess the ability to move away from discrete lab-based sensing methods, advancing toward personalized, convenient therapy.

\subsection{Nanomaterials employed in electrochemical detection of wound biomarkers}

Different sensors based on nanocomposite materials have been designed for electrochemical bio-analyte detection. This has been seen to significantly improve sensing performance in terms of sensitivity.[119] Conductive substrates made functional with infused nano-material, have been seen to improve sensitivity through superior conductivity, higher electroactive area and surface-volume ratios. This facilitates accelerated electron transfer between the analyte and the transducer on account of increased binding sites and surface interactions. An overview of the different materials explored in enzymatic UA detection is illustrated in Table 2.2, with a summary of the sensing performance. 
Different metal $(\mathrm{Pt} / \mathrm{Cu} / \mathrm{Zn} / \mathrm{Ag} / \mathrm{Ca} / \mathrm{Sn} / \mathrm{Au} / \mathrm{Al})$ and metal-oxide $(\mathrm{ZnO})$ based nanomaterials have enabled superior sensing performance in UA and Xanthine detection.[120-124] The offered large surface area from distribution of the nanomaterial across the electrode surface, increases electrical conductivity and electrocatalytic properties of the surface.[125-127] This provides significant mechanical strength to facilitate rapid electron transfer between the analyte and the transducer. [6] Platinum-nickel (Pt-Ni) nanocomposites and carbon based metal $(\mathrm{Co} / \mathrm{Ni} / \mathrm{Ce} /)$ nanocomposites have also

\begin{tabular}{|c|c|c|c|c|c|c|}
\hline Enzyme matrix & Technique & Stability & $\begin{array}{c}\text { Linear } \\
\text { Range } \\
(\boldsymbol{\mu M})\end{array}$ & $\begin{array}{c}\text { Detection } \\
\text { Limit } \\
(\mu M)\end{array}$ & $\begin{array}{l}\text { Sampling } \\
\text { matrix }\end{array}$ & $\mathbf{R}$ \\
\hline $\begin{array}{l}\text { UOx-carbon- } \\
\text { polyester }\end{array}$ & Amperometry & $7 \mathrm{~h}$ (RT) & $0-800$ & 100 & $\begin{array}{l}\text { Simulated } \\
\text { wound } \\
\text { fluid }\end{array}$ & [71] \\
\hline $\begin{array}{c}\text { chitosan-UOx- } \\
\text { BSA+glutaraldehyde- } \\
\text { PrussianBlue-carbon }\end{array}$ & $\begin{array}{c}\text { Chronoampero } \\
\text { metry }\end{array}$ & $8 \mathrm{~h}(\mathrm{RT})$ & $\begin{array}{l}100 \\
800\end{array}$ & 100 & - & [13] \\
\hline $\begin{array}{c}\text { polyurethane-UOx- } \\
\text { xerogel (THF/silane)- } \\
\text { Pt }\end{array}$ & Amperometry & 10 days (RT) & $\begin{array}{l}100- \\
700\end{array}$ & $<10$ & - & [72] \\
\hline $\begin{array}{c}\text { UOx-glutaraldehyde - } \\
\text { ZnO-carbon }\end{array}$ & Amperometry & - & $\begin{array}{l}200- \\
800\end{array}$ & 200 & Blood & [73] \\
\hline $\begin{array}{c}\text { PPD-UOx- } \\
\text { BSA+glutaraldehyde- } \\
\text { PrussianBlue- } \\
\text { graphite-Ag/AgCl- } \\
\text { PET }\end{array}$ & $\begin{array}{c}\text { Chronoampero } \\
\text { metry }\end{array}$ & 4h (RT) & $\begin{array}{c}50- \\
1000\end{array}$ & 50 & Saliva & [74] \\
\hline $\begin{array}{l}\text { UOx-glutaraldehyde- } \\
\text { PrussianBlue- } \\
\text { graphite }\end{array}$ & $\begin{array}{c}\text { Chronoampero } \\
\text { metry }\end{array}$ & $\begin{array}{c}90 \% \text { after } 15 \\
\text { days (RT) }\end{array}$ & $30-300$ & 10 & Serum & [75] \\
\hline $\begin{array}{l}\text { UOx-methionine-Au- } \\
\qquad \mathrm{Cu}\end{array}$ & $\begin{array}{c}\text { Cyclic } \\
\text { voltammetry }\end{array}$ & $\begin{array}{c}89 \% \text { after } \\
100 \text { days } \\
\text { (RT) }\end{array}$ & $0-2380$ & 2.4 & Unine & [76] \\
\hline
\end{tabular}

Table 2.2 Comparison of materials employed in enzymatic Uric acid determination using UOx functionalized electrochemical biosensors. RT represents room temperature [236] 
been explored in UA detection,[128-130] offering linear, reproducible response with good specificity. The incorporated nanomaterials offered superior catalytic activity and enhanced sensitivity with lower detection limits, as displayed by the study.

Most acclaimed materials used in UA detection involve nanoparticles of carbon (C) and gold $\mathrm{(Au}$ ). Synthesis of specific nanostructures (dots, fibers, tubes, wires, rods, sheets, etc.) $[128,131-135]$ from graphene and graphene oxide (GO), are known to enhance electrochemical sensing response. Such nanomaterials have also been reported to enable enhanced response in Xanthine detection offering rapid response in less than 2 min.[136] Single-walled carbon nanotubes (SWCNT), double-walled carbon nanotubes (DWCNT) and multi-walled carbon nanotubes (MWCNT) have been explored in detection of Xanthine and noted to record linear response in concerned physiological ranges.[137-138]

Gold nanoparticles (AuNP) are widely used to allow for improved detection through increased surface interactions and higher surface-volume ratios. Exhibiting good biocompatibility, its inclusion in the design of a biosensor provides a suitable microenvironment for biosensing. In addition to offering increased conductivity, it assists in maintaining the redox potential of the undergoing reaction with good electrochemical stability. With portrayed good electrical properties, presence of $\mathrm{Au}$ nanocomposites facilitates enzymatic detection, reducing the insulating effects of involved proteins to enable direct electron transfer through a conductive channel.[139-140] Specific structures of AuNP have been noted to reduce the thickness of the double layer formed on the transducer in detection, and offer improved electron transfer from reduced distance between the transducer and the analyte. Nanocomposites of carbon and gold have also been reported to enable detection of Xanthine at lower potentials.[141-142] 
Specific structure and morphology of such material at the nanoscale enables superior sensitivity owing to augmented ionic interactions offered from large surface-volume ratios. Such materials are reported to improve sensing performance and enable detection at specific potential in relevant physiological ranges.

\subsection{Substrate materials explored in wearable biosensing}

Different flexible materials have been explored in the design of wearable biosensors. Ongoing attempts in the synthesis of flexible biomaterials target the use of stretchable materials that are biocompatible and conformal to the skin surface, so that they can be integrated into body-borne form factors. Various configurations for wearable sensor designs have been conceptualized for collecting valid information from different physiological parameters (heart and breath rate, wrist pulse, facial expression, vocalization and metabolism). However, their development in the medical space has been slow and their reliability for continuous monitoring in real-time is still an unconquered challenge. This can be attributed to the consideration of several factors involving biocompatibility, compactness with skin, durability, size and weight.

The implementation of health solutions in convenient and economical formats has been marked by the advent of flexible, soft materials. Paper based analytical tools (glucose strips/pregnancy strips) are being explored as an attempt to shift lab-based diagnostics toward POC.[143] Organic membranes of carbon or other metals demonstrate stretchable properties, which can be employed in the detection of limb/body motion. These, however, do not provide a comfortable setting to translate human gestures from related measurements of pressure or strain. Tape and tattoo-based sensors have been investigated to identify specific facial expressions from muscle movements. Biocompatible and 
mechanically invisible tattoo electronics, explored as an epidermal platform for high performance electrical measurements, have offered repeatable, continuous measurements in wellness management.[16]

Attuning the electrical and mechanical properties of such different kinds of conducting and semiconducting materials allow for improved stretchability and electrical conductivity in seamless and robust transduction of physiological parameters. Flexible polymer materials, among others, are investigated because of their immensely adjustable physiochemical properties. PDMS is a widely used silicone elastomer which is used as wearable substrate material, due to its biocompatibility and low elastic modulus. Its nontoxic, non-flammable and hydrophobic properties, along with good processability, has allowed its use in wearable sensing devices.[16] Other flexible elastomers that are gaining popularity are rubber-based silicone in EcoFlex, DragonSkin and Silbione, which are known for their use in flexible implantable sensors due to their biocompatibility and chemical inertness. These have been reported to show superior deformability and conformability on surfaces with varied textures and geometries, rendering their compliance to wearable biosensing.[3]

Polycarbonate (PC), polyethylene (PET) and polyimide (PI) films are also gaining diversity as substrate materials because of their high stretchability, thermal stability and ease of printability.[3,144] Other organic polymer materials and co-polymers involving poly (3,4-ethyl-enedioxythiophene-poly(styrene-sulfonate) $\quad$ (PEDOT:PSS), Polyvinylidene fluoride (PVDF), Polytetrafluoroethylene (PTFE), Polyaniline (PANI) and polymethyl methacrylate (PMMA), are investigated as solid-state physical sensing platforms owing to their tunable electro-mechanical properties, thermal stability and 
electrochemical inertness. Liquid-state materials are also being examined for designing flexible diagnostic platforms, with exciting applications in personalized health analyses. Utilizing the signal transduction mechanism from mechanical deformations, this employs ionic liquids in the form of metallic alloys from eutectic mixtures of gallium-indium (eGaIn) and Galinstan.[145] This has allowed for advancements in numerous biochemical assays due to their superior sensing performance from low sample volumes, reduced fabrication costs and low power requirements. They claim to offer a more robust platform for wearable biosensing, as fundamentally liquids are more deformable than solids.[145]

To promote wearability, efforts are directed to cater to the bending, stretching and twisting of joints and skin which can induce curvature related variations to the response. Fabric and cellulose based material like cotton and other textiles are being investigated as platforms in wearable health monitoring. Conductive textiles are being adopted in apparel like gloves, socks and other clothing to demonstrate their potential in wearable diagnostics.[3, 146-147] Significant research is conducted to integrate conductive fibers such as thin filaments or metallized textile yarns, which can be woven, knit or sewn onto consumable wearables. Such forms of electronic textiles are gaining popularity owing to their versatility and robustness to sustain mechanical variations. These materials can be engineered to miniaturized bendable form factors for the potential integration of sensors onto a wearable construct. This attempts to achieve conformal contact on skin-like curvilinear surface of the human body for personalized wellness management at POC.[148]

\subsection{Design of wearable biosensors}

Different flexible sensing platforms have been explored in enzymatic electrochemical detection of biomarkers. Advancements are being examined with flexible interconnects 
and fibers used in conjunction with graphene based functional materials[149] using different fabrication techniques like drawing or printing.[150] Among the different methods used to fabricate electrodes on textile based material, screen-printing is among the most widely used, easier methods. Other procedures include inkjet-printing, roll-to-roll printing, gravure printing and laser direct write printing. An overview of these methods is elucidated below:

Screen printing - This is a method, where printing on a substrate, is achieved using a screen of specific mesh size to achieve required resolution. This allows transfer of ink onto a substrate, excluding the areas which have been masked from it using a stencil. A squeegee is used to fill the open mesh apertures with ink, while the mesh screen is in contact with the substrate.[151] Screens are usually made of polyester or stainless steel. The selection of material and mesh count are important for obtaining optimal resolution in printing, based on the particle size and composition of ink used.

Inkjet printing - Inkjet printing is a form of digital printing and is used in digital graphic design, whereby a digital image is created by propelling ink drops onto the substrate. Inkjet printers use solvent-based inks from volatile organic compounds (VOCs) that are cured when exposed to UV light. They provide the advantages of cost-effective printing and good durability. However, use of solvents in their composition increases diffusion capability of the ink when it is used for printing on flexible textile material.[152]

Roll-to-roll printing - Widely known as roll-to-roll processing, this method is used for flexible electronic devices and entails printing or applying coatings on a roll of flexible material. Thin film based electronic circuits can be patterned onto large, portable substrates using this method.[153] 
Gravure printing - This kind of printing is a high speed roll-to-roll printing method which involves engraving an image onto an image carrier, acting as a rotary imaging press. The image carrier transfers ink to an impression roller, which then transfers it onto desired areas of the substrate. The substrate is sandwiched between the carrier and the impression roller, ensuring proper contact of the substrate with the carrier from applied force of the roller. This ensures even and maximum coverage of ink for a uniform coating. [154] Laser printing - Laser printing is an electrostatic digital printing method which can produce high quality text and graphics from a laser beam. The beam is passed over a charged image carrier which selectively collects electrically charged ink particles and transfers it onto the substrate. This is followed by heating to affix the print onto the substrate. Using the principle of photoconductivity, the ink particles are attracted to the carrier, transferring the image onto the substrate by direct contact using raster printing. [155]

For wearable wound diagnostics, detection of biomolecules is explored on soft, wearable, healing material like cotton gauze. Feasibility of biomarker detection from such a platform can be employed in personalized wound diagnostics for continual management of healing, as and when necessary. Such design shall eliminate the unnecessary paraphernalia of bulky equipment, which make it non-conformal to damaged skin. Electrochemical characterizations have been conducted on nanomaterial based enzymatic sensors designed to quantify changes in UA and Xanthine concentrations from different biofluids of injured patients. 


\section{CHAPTER 3}

\section{ENZYMATIC BIOSENSOR FOR STABLE DETECTION OF URIC ACID BY ENTRAPMENT IN PVA-SbQ POLYMER MATRIX}

In this chapter, electrochemical approaches using UOx as an enzyme to detect UA for wound monitoring have been reported. UOx was entrapped in a cationic polymer matrix, poly (vinyl alcohol) N-methyl-4(4'formylstyryl) pyridinium methosulfate acetal (PVA$\mathrm{SbQ}$ ), and response stability over multiple days was assessed. Stability of enzymatic detection under physiologically relevant variations of temperature and $\mathrm{pH}$ were explored from the enzyme entrapped in the polymeric matrix. A redox electron shuttle, ferrocene carboxylic acid (FCA) was utilized to facilitate accelerated electron transfer and investigate electrochemical performance. Sensor response was recorded using voltammetric techniques to measure UA within physiologically relevant ranges.

\subsection{Enzyme Uricase, UOx}

UOx is an enzyme that plays an integral role in the catabolic metabolism pathway of humans. With no metal or organic factor required for catalysis, UOx is made of 303 amino acid residues, 15 of which are involved with the active site.[156] Among these residues, Leu170 forms a hydrophobic floor beneath the substrate and plays a role in its positioning. Three of the conserved residues (Arg176, Val227, and Gln228) within each active site act as ligand tweezers to bind and hold the substrate in place. Within the active site, Asn254 from one monomer and Thr57 from another hydrogen, bond a single water molecule,[157] assisting in specific UA oxidation.[115] Selective oxidation of UA occurs in four identical active sites of the enzyme's quaternary structure, located at the interfaces between its four subunits.[158] 


\subsection{Effect of physiological pH on Uricase}

A key factor that influences wound healing is $\mathrm{pH}$ of the area surrounding the bruise. Chronic non-healing wounds are known to have an alkaline $\mathrm{pH}$ (7.15 to 8.9). As healing progresses, the $\mathrm{pH}$ becomes more acidic, approaching that of normal skin (4-6). The $\mathrm{pH}$ of different biofluids namely blood, serum, urine, saliva and sweat are slightly acidicneutral $(5-7)[74,159]$. Since enzymes can only function in a specific $\mathrm{pH}$ environment; it was necessary to assess the activity of UOx in various $\mathrm{pH}$ environments. This can also provide an understanding of how a wound's severity changes with time.

Enzyme UOx is known to be active in alkaline environments between $\mathrm{pH} 8$ and 9, with lowered activity as it deviates beyond. To evaluate the effect of $\mathrm{pH}, \mathrm{UOx}$ was studied from pH 5 to 10 using absorption spectroscopy. Specific activity of UOx was calculated from the absorption values and plotted in Figure 3.1. Higher activity of UOx $\left(4.3 \mathrm{U} \mathrm{mg}^{-1}\right)$ was recorded at $\mathrm{pH} 7.8$ which elevated response in the $\mathrm{pH}$ region between 7 and 9 . Electrochemical analyses were performed over different $\mathrm{pH}$ to assess the change in

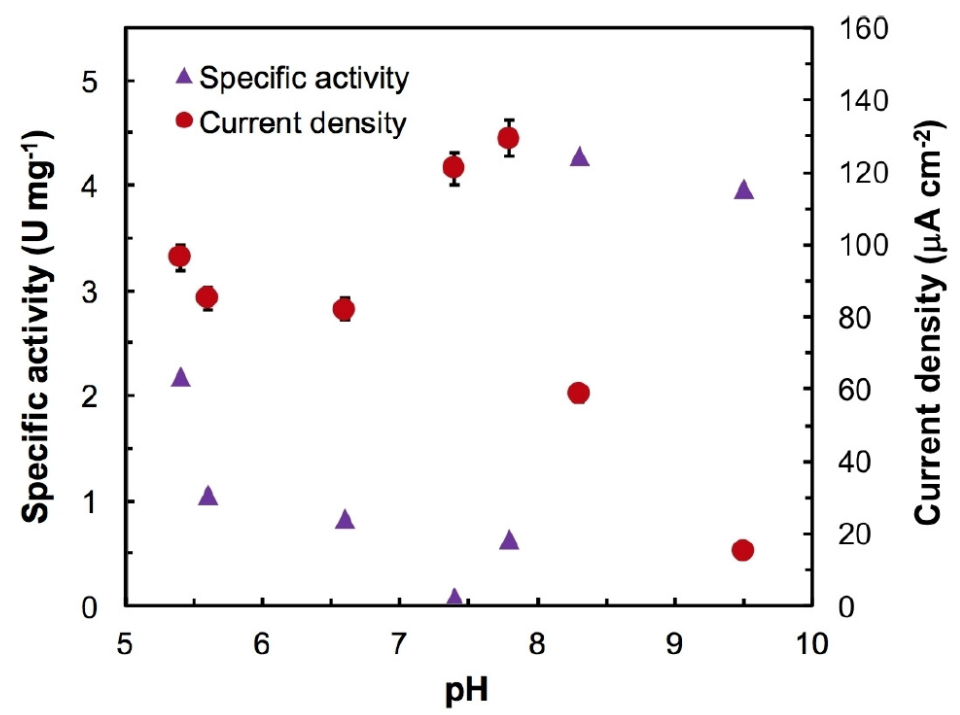

Figure 3.1 Comparison of $\mathrm{pH}$ effect on specific activity of UOx and current density of UOx electrode [236] 
response of UOx electrode. As noted from Figure 3.1, higher current density of about 120$130 \mathrm{~mA} \mathrm{~cm}{ }^{-2}$ was observed between $\mathrm{pH} 7$ and 9, beyond which lower response was attained. These investigations provide an improved understanding of the $\mathrm{pH}$ environments suited for enzymatic UA detection. With higher response obtained at $\mathrm{pH} 7.8$, this was chosen as the optimum $\mathrm{pH}$ for subsequent studies using a UOx based sensor, described in the following sections.

\subsection{Enzyme immobilization protocols in detection of Uric Acid}

In enzyme electrochemistry, the immobilization technique determines enzyme loading and stability. Existing immobilization approaches include physisorption, entrapment, encapsulation and crosslinking.

Physisorption provides for a simple and cost-effective method in direct immobilization. Although, it shields agglomeration and provides for uniform loading across the sensor surface, the enzyme affixes through hydrogen bonds, electrostatic and weak van der Waal's interactions. This could lead to leaching of the enzyme and reduced enzyme activity.

Entrapment is a method where the enzyme is confined within a certain hydrogel or polymeric matrix. It is entrapped through covalent and non-covalent bonds offering improved stability, reduced denaturation, retainment of enzyme activity and its electrocatalytic properties.

Encapsulation involves enzyme enclosure within a semi-permeable polymer membrane. Although this provides stable response and good repeatability, the thin interface can cause enzymes to leach.

Crosslinking entails immobilization of the enzyme with the help of a suitable crosslinker which firmly binds the enzyme onto the transducer surface through covalent bonds. 
However, this method does not allow for increased loading and can alter enzyme orientation while binding with linker molecules, with conformational changes at the active site. Such structural changes at the active site can cause response to degrade from lower enzyme activity and leaching.

In enzymatic UA detection, entrapment and encapsulation of the enzyme UOx have been investigated using different polymeric matrices of PANI, polypyrrole and PVC,[160163] or gels like chitosan, silica and collagen.[88, 164-165] Ionic polymers have also been reported to provide enhanced sensitivity and stability.[166-167]

\subsubsection{Uricase entrapment in PVA-SbQ polymer matrix}

In this work, enzymatic electrochemical sensors in which UOx was either physisorbed or entrapped in PVA-SbQ, a cationic polymer, were investigated in UA detection. The amine group of the styrylpyridinium side chains in the PVA backbone offers firm attachment to immobilize the enzyme to the electrode surface.[168-170] This is due to the net charge of the enzyme being partially negative $\left(\delta^{-}\right)$above $\mathrm{pH} 7.5$. The $\mathrm{pKa}$ and the

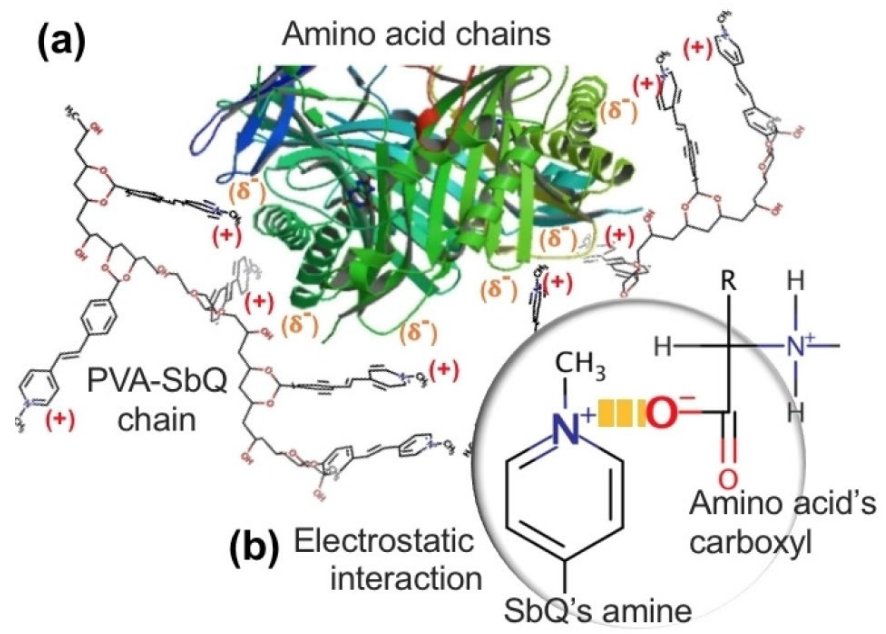

Figure 3.2 (a) PVA-SbQ interaction with negatively charged amino acid chains of UOx enzyme, (b) Inset depicts structural interactions between the carboxylic functional group of the enzyme and the amine side chains of the polymer [236] 
isoelectric point values of UOx are 4.64 and 7.5 respectively.[163] The binding and interaction of the amino acid chains of UOx with the polymer is depicted in Figure 3.2 a and $b$, with the polymer structure in the schematic having minimized bond energy.

Use of PVA-SbQ for improved stability and shelf life of UOx based enzymatic biosensors have been investigated in this study. This biocompatible polymer has an edge over others with its protective effect and the advantage of providing biosensors with a longer lifetime.[171-172] Stability studies for PVA-SbQ showed that the polymer is stable over multiple days (Figure 3.3).

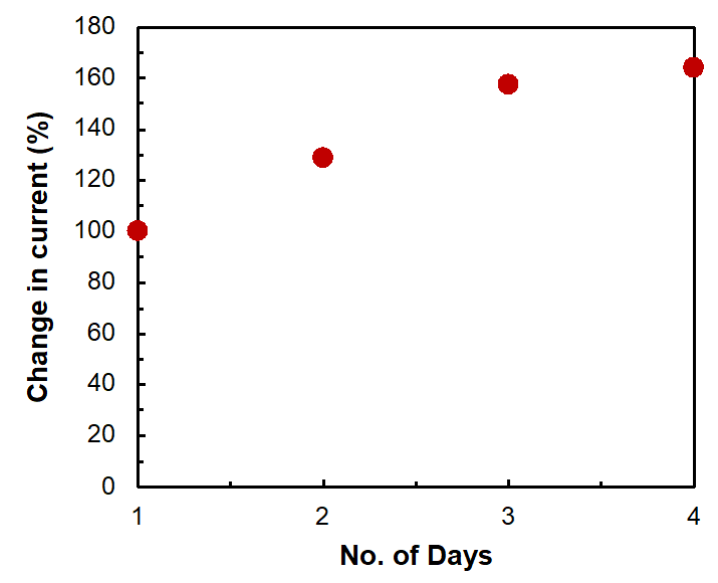

Figure 3.3 Plot representing stability of the pristine PVA-SbQ obtained from continuous measurements for $\mathrm{n}=4$ over multiple days with RSD of 0.41 [236]

\subsection{Ferrocene Carboxylic Acid as a mediator in Uric Acid detection}

It is known that enzymes have deeply buried active sites and diffusion of reagents to the electrode surface can be limited.[173] As electron transfer decays exponentially with increased distance,[171] weaker response may be obtained for lower concentrations. In the design of second-generation biosensors, this limitation is being overcome with the use of mediators which provide improved response from the reaction on the transducer. The use of a redox electron shuttle as a mediator in enzymatic UA detection facilitates improved 
electron transport between the active site of the enzyme and the electrode, offering enhanced response. Such mediators include derivatives of benzoquinone, ferricene/ferrocene and methyl/methoxy compounds.[174-176] Ferri/ferro based compounds require lower energy for the redox reaction, enabling detection in a lower potential window with minimized interference from other electro-active species. Electrochemical voltammetric investigations have shown ferrocene and its derivatives (ferricinium ion forms) to be electron acceptors for specific enzymes and molecules.[177] This addresses the limited diffusion rates seen in first generation biosensors, providing improved signal with minimized interference from dissolved oxygen in produced $\mathrm{H}_{2} \mathrm{O}_{2}$. Ferrocene Carboxylic Acid (FCA) has been used in this study as a mediator to aid accelerated electron transfer between the active site of the enzyme and the transducer surface.

\subsection{Experimental}

\subsubsection{Materials}

Lyophilized UOx powder containing 15-30 $\mathrm{U} \mathrm{mg}^{-1}$ of UOx was purchased from Sigma Aldrich. The electron transfer mediator FCA was purchased from Chem-Impex International, Inc. PVA-SbQ purchased from Polysciences, Inc., was used to entrap UOx on standard screen-printed carbon electrode (SPCE) purchased from CH Instruments, Inc., United States. UA, $\mathrm{H}_{2} \mathrm{O}_{2}$, sodium phosphate monobasic $\left(\mathrm{NaH}_{2} \mathrm{PO}_{4}\right)$ and sodium phosphate dibasic $\left(\mathrm{Na}_{2} \mathrm{HPO}_{4}\right)$ and boric acid used were of analytical grade. All aqueous solutions were prepared using deionized (DI) water. Phosphate buffer (20 mM) and boric acid (20 $\mathrm{mM}$ ) were used as electrolyte solutions. Phosphate buffer solutions (PBS) (pH 5 to 7.8) were prepared using $\mathrm{NaH}_{2} \mathrm{PO}_{4}$ and $\mathrm{Na}_{2} \mathrm{HPO}_{4}$ salts. Boric acid buffer solutions were 
prepared for $\mathrm{pH} 8$ to $\mathrm{pH} 10$ solutions. $0.2 \mathrm{U} \mathrm{ml}^{-1}$ horseradish peroxidase (HRP) and $50 \mathrm{mM}$ Amplex red reagent, of analytical grade were used from ThermoFisher Scientific, for fluorescence studies. All other chemicals used were of analytical grade.

\subsubsection{Apparatus and Methods}

Cyclic voltammetry (CV) and differential pulse voltammetry (DPV) were performed using the $\mathrm{CHI}-230 \mathrm{~B}$ potentiostat from $\mathrm{CH}$ Instruments, Inc. Electrochemical characterizations of the enzymatic electrode were carried out in a conventional three electrode cell assembly consisting of $\mathrm{Ag} / \mathrm{AgCl}$ as reference electrode and $\mathrm{Pt}$ wire as counter electrode. The working electrode was either bare SPCE or modified SPCE. The SPCEs were modified with (i) PVA-SbQ (polymer), (ii) UOx (physisorbed), and (iii) UOx in PVASbQ (entrapped).

Effect of pH variations on enzyme activity was analyzed using Evolution 201 UV Visible Spectrophotometer, ThermoFisher Scientific. Material characterization studies of the electrode surface were performed using Raman spectroscopy with a $514.5 \mathrm{~nm}$ Ar laser, and impedance measurements were conducted with Gamry impedance analyzer. Scanning electron microscope (SEM) JEOL 6330, ThermoFisher Scientific and Nikon C1 confocal microscope (Nikon Instruments Inc., Melville, NY) were used to obtain images of the enzymatic sensor surfaces. This provided an understanding of optimized polymer-enzyme loading for robust immobilization toward improved stability. Entrapped sensor response was investigated over multiple days under physiologically relevant variations of $\mathrm{pH}$ and temperature. Electrochemical analyses were conducted to study repeatability, reproducibility and shelf life of the entrapped sensor. 


\subsubsection{Enzyme Immobilization}

Prior to immobilization, SPCEs were polished with $0.05 \mathrm{~mm}$ alumina slurry and rinsed thoroughly with DI water. PVA-SbQ solution was prepared with DI water in a ratio of 2.5:0.5 [V/V], while $0.5 \mathrm{U} \mathrm{cm}^{-2} \mathrm{UOx}$ was prepared in $1 \mathrm{ml} \mathrm{PBS} \mathrm{buffer} \mathrm{solution} \mathrm{(pH} \mathrm{7.8).}$ All enzyme immobilizations were carried out by drop casting, nitrogen drying, and then vigorously rinsing in buffer prior to testing. Enzyme loading studies were carried out by keeping the volume of PVA-SbQ constant and varying UOx [V/V] in the ratio of 1:2 (53.2 $\left.\mu \mathrm{g} \mathrm{cm}^{-2}: 0.25 \mathrm{U} \mathrm{cm}^{-2}\right), 1: 4\left(53.2 \mu \mathrm{g} \mathrm{cm}^{-2}: 0.5 \mathrm{U} \mathrm{cm}^{-2}\right)$ and $1: 6\left(53.2 \mu \mathrm{g} \mathrm{cm}^{-2}: 0.75 \mathrm{U} \mathrm{cm}^{-2}\right)$. Vigorous rinsing was done to remove excess unbound enzyme. Drying in nitrogen enables removal of excess water from the electrode surface, forming a gel like structure. These immobilization steps were carried out in an ice box to prevent enzyme denaturation and leaching. In physisorption, $0.25 \mathrm{U} \mathrm{cm}^{-2} \mathrm{UOx}$ was drop casted on the bare SPCE and dried in nitrogen in an ice box for $10 \mathrm{~min}$. For fluorescence studies, the electrode was immobilized with polymer and UOx in different ratios as discussed. $20 \mathrm{U} \mathrm{ml}^{-1} \mathrm{HRP}$ was then immobilized on the electrode in the polymer UOx matrix. In the presence of $1 \mathrm{mM}$ UA with $50 \mathrm{mM}$ amplex red, fluorescence was investigated at $570 \mathrm{~nm}$.

\subsection{Entrapped enzymatic biosensor surface characterization}

UOx entrapped in PVA cationic polymer on the electrode was characterized by spectroscopic, optical and electrochemical techniques to assess the sensor surface morphology and characteristic behavior. Physisorbed UOx on the electrode surface was used as control in these studies. To obtain highest loading of UOx on the electrode surface, various ratios of polymer and $\mathrm{UOx}(1: 2,1: 4$ and 1:6) were studied. 
Raman spectra of proteins consist of bands associate with its peptide chains. This technique offers several advantages for analysis of enzymes through the shifts in peak and intensity. The structural fingerprint of specific molecules in entrapped UOx-PVA-SbQ was assessed using a continuous wave $(\mathrm{CW})$ argon ion $(\mathrm{Ar}+)$ laser (model 177G02, Spectra Physics) of $514.5 \mathrm{~nm}$ excitation. The spectrum exhibited in Figure 3.4a depicts two main zones at intermediate $\left(1,100-1,400 \mathrm{~cm}^{-1}\right)$ and high $\left(1,500-1,700 \mathrm{~cm}^{-1}\right)$ frequencies. While a polymer functionalized electrode provided typical peaks at $1330.8 \mathrm{~cm}^{-1}$ and $1613.72 \mathrm{~cm}^{-}$ ${ }^{1}$ due to $-\mathrm{CH}$ and $-\mathrm{OH}$ interactions, amine in the pyridine of PVA-SbQ showed bands at $1184.34 \mathrm{~cm}^{-1}$ and $1214.34 \mathrm{~cm}^{-1}$. Minute shifts in these peaks were observed for UOx functionalized electrodes, with the polymer chain interactions noticeably overshadowing the amino acid chain interactions of UOx. The entrapped enzymatic electrode provided distinct peaks for amide III band of UOx and phenylalanine at $1324 \mathrm{~cm}^{-1}$ (Figure 3.4b) and $1116.99 \mathrm{~cm}^{-1}$ (Figure $3.4 \mathrm{c}$ ) respectively. This can be attributed to C-C and -C-O-C bonds and changes in the $\alpha$-helical structure and $\beta$-sheets of the enzyme due to electrostatic
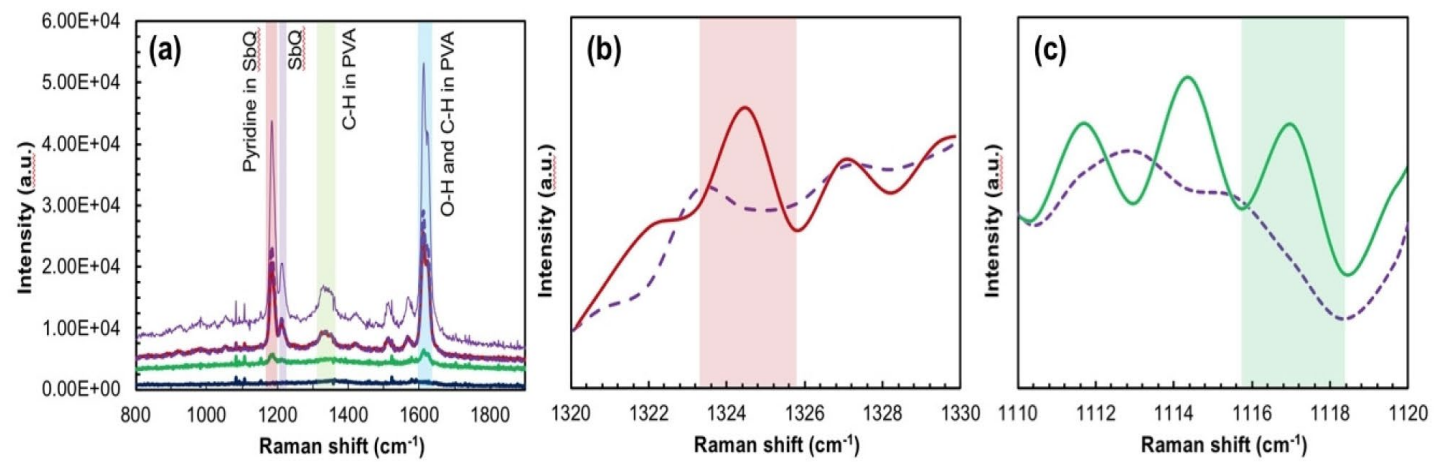

Figure 3.4 Raman spectra of UOx functionalized substrates measured with $514.5 \mathrm{~nm}$ Ar laser. (a) Polymer-UOx ratio 1:2 in (-) red line, 1:4 in (- - ) dashed purple line, 1:6 in (-) green line, UOx physisorbed in (-) dark blue line and pristine polymer in (-) purple line. The red, purple, green, blue bands represent the groups in PVA-SbQ. (b) amide III (red band) and (c) phenylalanine (green band) groups in polymer-UOx functionalized substrate [236] 
interactions between $\delta^{-}$of the amino acid chains and positive charge of pyridine in PVASbQ. As compared to physisorbed UOx, a slight shift of the amide I band is seen from $1578.78 \mathrm{~cm}^{-1}$.

Other amino acid residues like leucine, valine and arginine also provided smaller intensity peaks at 772,1037, 1339 and $1569 \mathrm{~cm}^{-1}$ representing stretching vibrations of $\mathrm{CH}$, $-\mathrm{NH}$ and $-\mathrm{CN}$ bonds of the carboxyl and amino groups in the UOx functionalization matrix (Figure 3.5). These results conform with those previously examined for these groups [178-180]. Copper, being embedded within the amino acid chains, displayed a relatively small peak around $985.7 \mathrm{~cm}^{-1}$.[181] In Figure 3.5a there was a decrease in intensity of the predominant polymer peaks as the UOx volume increased (polymer: UOx ratio in electrode
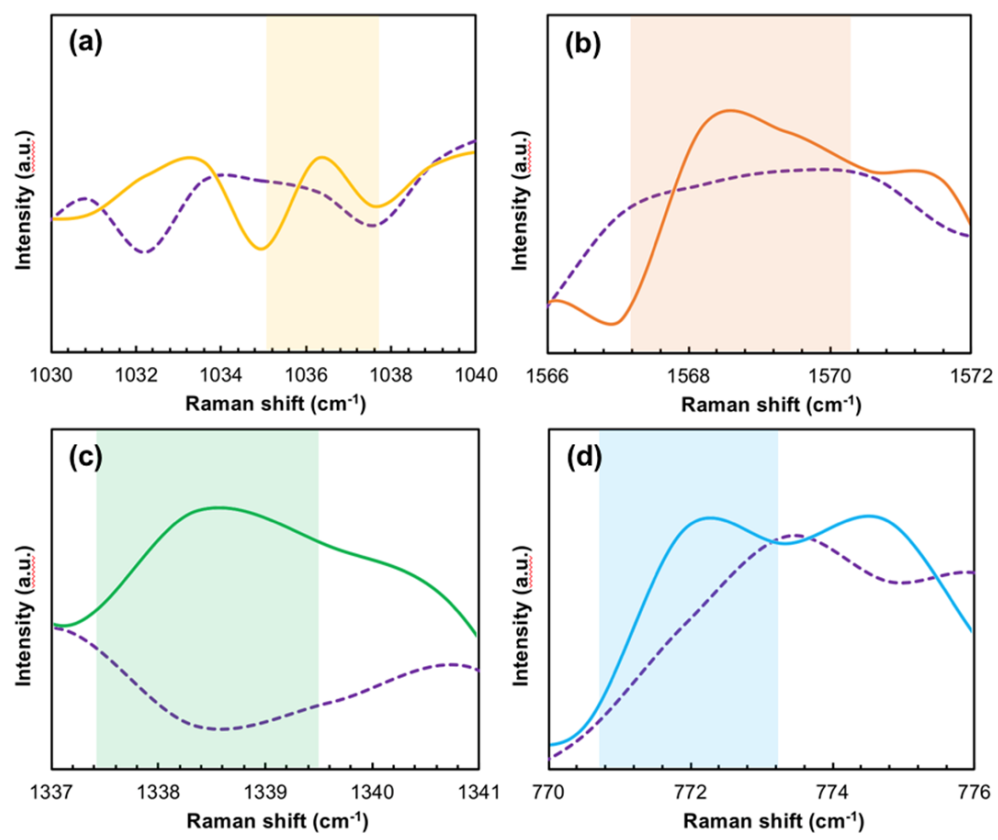

Figure 3.5 Raman spectra of UOx functionalized substrate measured with $514.5 \mathrm{~nm} \mathrm{Ar}$ laser, showing peaks of amino acid residues depicting bonds of (a) $\mathrm{C}-\mathrm{C}$ (yellow band) and (b) $\mathrm{CO}_{2}{ }^{-}$(orange band) in Arginine; (c) $\mathrm{C}=\mathrm{C}$ and $-\mathrm{CH}_{3}$ in Valine (green band); and (d) $-\mathrm{CO}_{2}{ }^{-}$(blue band) in Leucine. The dashed purple line is the signal from pristine PVA-SbQ [236] 
preparation). This decrease was due to reduced polymer concentration on the electrode surface.

Fluorescence microscopy was performed to elucidate the morphology of the UOx entrapped matrix and to visualize the UOx reaction. Along with physisorbed, the effect of three different enzyme ratios of 1:2; $1: 4$ and $1: 6$ in the hybrid PVA-SbQ/UOx system were investigated. In these studies, amplex red was used as a fluorescent tag for imaging immobilized enzyme on the electrode surface. On enzymatic oxidation of UA, amplex red reacted with the formed byproduct, $\mathrm{H}_{2} \mathrm{O}_{2}$ in the presence of HRP to form fluorescent product, resorufin at $570 \mathrm{~nm}$. Results (Figure 3.6) offered a pictorial representation of the entrapped enzyme on the electrode surface. Increased UOx volume (polymer enzyme ratio) from $2 \mathrm{~mL}(1: 2)$ to $6 \mathrm{~mL}$ (1:6) (Figure 3.6a-c), led to more un-entrapped enzyme leaching out during electrode preparation procedures suggested by the formed dark empty spaces, depicted by yellow arrows in Figure 3.6. In 1:4 and 1:6 ratios (Figure 3.6b and c), the enzymes were scarcely populated compared to the 1:2 ratio (Figure 3.6a). Leaching from higher $\mathrm{V} / \mathrm{V}$ ratios is accredited to reduced cation to anion ratio in 1:4 and 1:6 matrices as compared to 1:2. This did not allow for firm entrapment of the enzyme within the interface
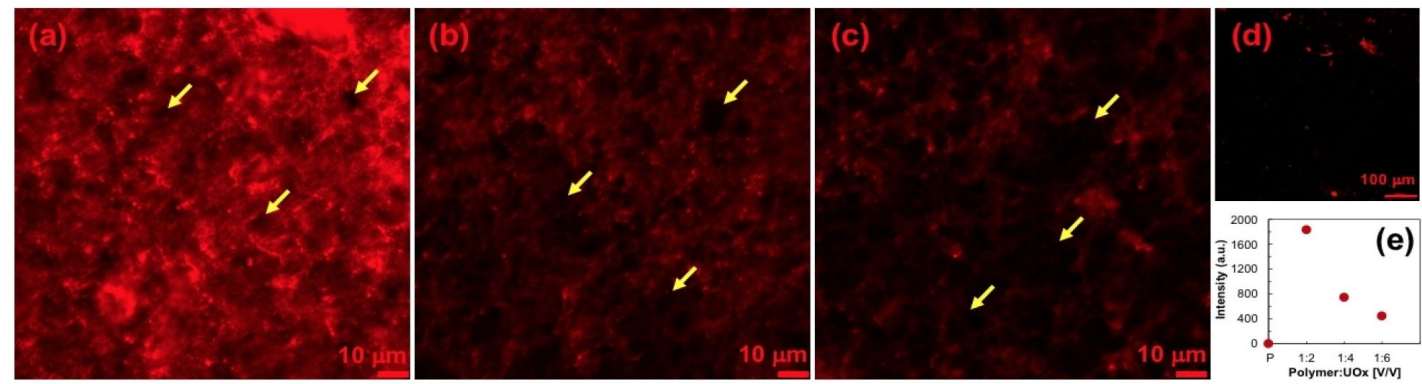

Figure 3.6 Fluorescence microscopic images of UOx functionalized substrates with various polymer-UOx ratios of (a) 1:2; (b) 1:4; (c) 1:6; Yellow Arrows indicate vacant spaces from reduced loading and low distribution of enzyme across sensor surface; and (d) physisorbed; (e) depicts comparison of mean intensities emitted at $570 \mathrm{~nm}$ from the UOx substrates [236] 
from lack of optimum distribution of enzyme on the electrode surface. Visibly more distinct fluorescence was observed with sharper intensity emission from the 1:2 polymer UOx ratio (Figure 3.6a). These images also suggest the active sites of enzymatic UA oxidation on the electrode surface from sites offering increased fluorescence. Fluorescence intensity was seen to decrease as the ratio of enzyme and polymer increased (Figure 3.6e and Figure 3.7) in the matrix. As compared to the entrapped sample, the physisorbed sensor surface (Figure 3.6d) portrayed very low or no emission at $570 \mathrm{~nm}$, indicating unstable immobilization without the polymer.

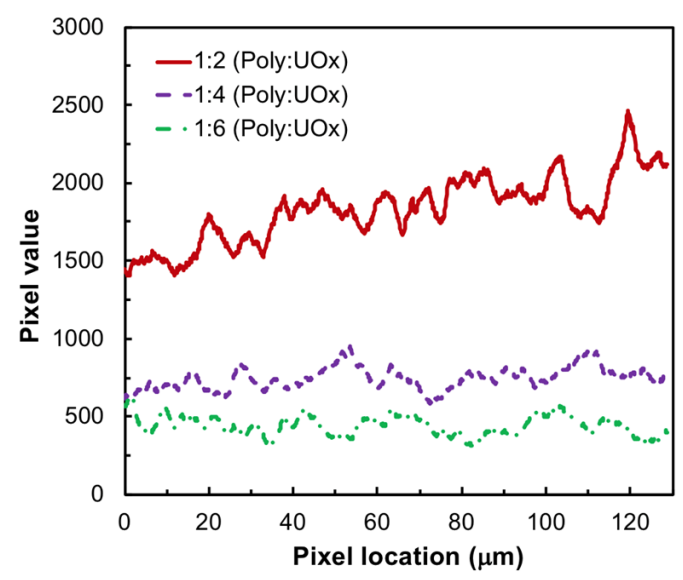

Figure 3.7 Plot depicting comparison of fluorescence intensities emitted from different UOx loaded substrates at $570 \mathrm{~nm}$. The mean pixel intensities of 1:2, 1:4 and 1:6 polymer-UOx substrates were $1830.89,742.64$ and 440.96 (a.u.) respectively [236]

These characteristics can also be observed from Scanning Electron Microscopy (SEM) images where morphology of distribution of the polymer UOx matrix on the working electrode (Figure 3.8) was seen. Topography of the physisorbed surface showed enzyme distribution with amorphous morphology on the electrode surface as seen in Figure 3.8a. The enzyme entrapped in the cationic polymer (Figure $3.8 \mathrm{~b}$ ) showed a more homogenous and smooth conformation, covered by the polymer coating. These imaging 
analyses provided visual evidence of surface functionalization for the immobilization matrix.

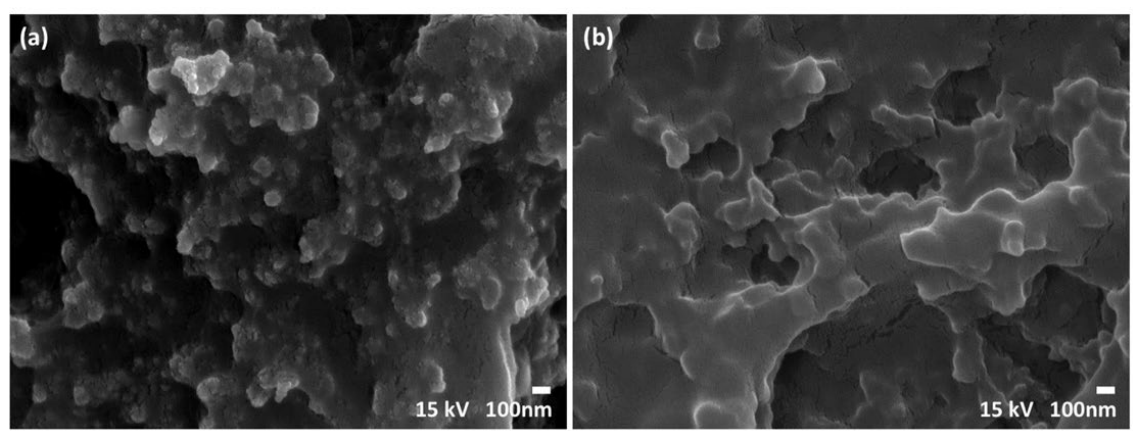

Figure 3.8 SEM micrographs of UOx (a) physisorbed and (b) entrapped functionalized substrates at $100 \mathrm{~nm}$ [236]

Electrochemical impedance studies (EIS) were carried out at $0.3 \mathrm{~V}$ from $1 \mathrm{mHz}$ to $100 \mathrm{kHz}$ in presence of the redox shuttle FCA to observe its influence on the resistive and capacitive effects of different enzyme loading in the functionalization matrices. UOx functionalized electrodes were tested using FCA redox probe $(20 \mathrm{mM})$. The Nyquist plot (Figure 3.9a) shows enhanced charge transfer resistance, $R_{\mathrm{ct}}$ (semicircle area) for lower polymer-enzyme ratio compared to the higher ratios $(20.47 \mathrm{M} \Omega$ to $0.20 \mathrm{M} \Omega)$ with error deviation within (+/-) 5.5\%. This increase in electron transfer resistance can be attributed to higher ratios of UOx in the matrix. As the thickness of the enzyme layer on the working electrode increased, less active sites are available on the electrode for the FCA redox reaction and electron transfer is decelerated. 

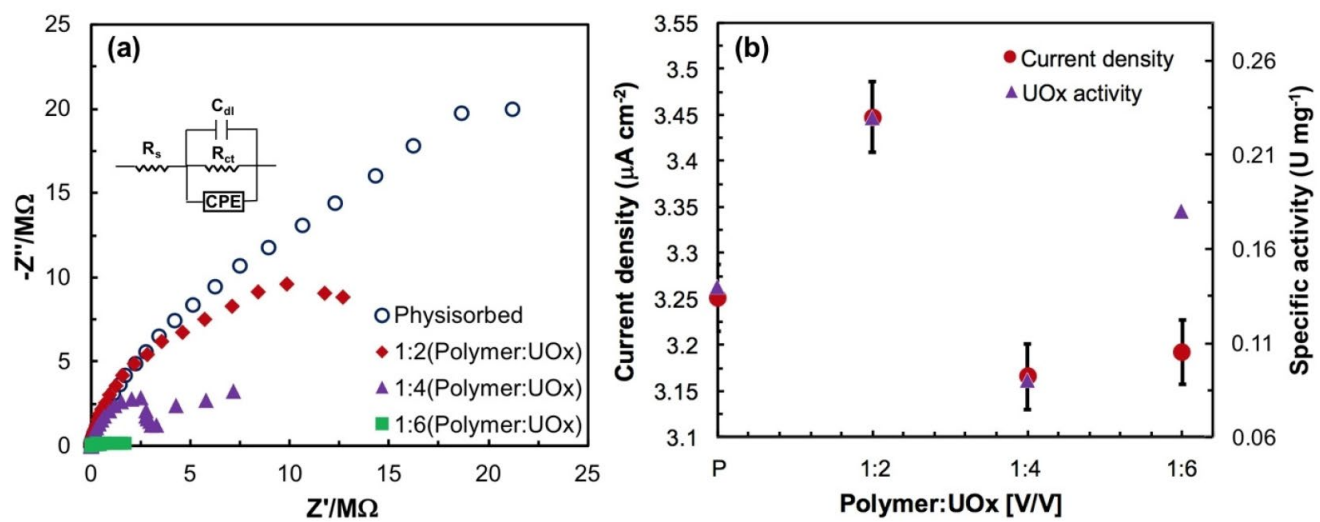

Figure 3.9 (a) Nyquist plot showing variation of impedance in presence of FCA at 0.3 $\mathrm{V}$ between $1 \mathrm{mHz}$ to $100 \mathrm{kHz}$ on functionalized substrates with varied loading; (b) Plot depicting change in UOx activity and current density with varied UOx loading [236]

The semicircle portion, observed at high frequencies correspond to electron-transfer limited process, with transfer of electrons between FCA and the electrode surface. A second inflection depicted in the plot, can be accredited to the presence of multiple capacitive effects as illustrated in Figure 3.9a. The straight-line portion represents diffusion limited ion transfer process at low frequencies.[182] $R_{\mathrm{ct}}$ is noted to increase with increased thickness of polymer-enzyme matrix on the electrode. Negligible variation in solution resistance $\left(R_{\mathrm{S}}\right)$ was noted among them. Impedance patterns were noted to change until 2.5 $\mathrm{M} \Omega$ at lower frequencies; while at higher frequencies, beyond $2500 \mathrm{kHz}$, with increased thickness of the film on the electrode, the change becomes less significant. The Bode plot (Figure 3.10) obtained from these impedance studies shows increase in phase angle with 

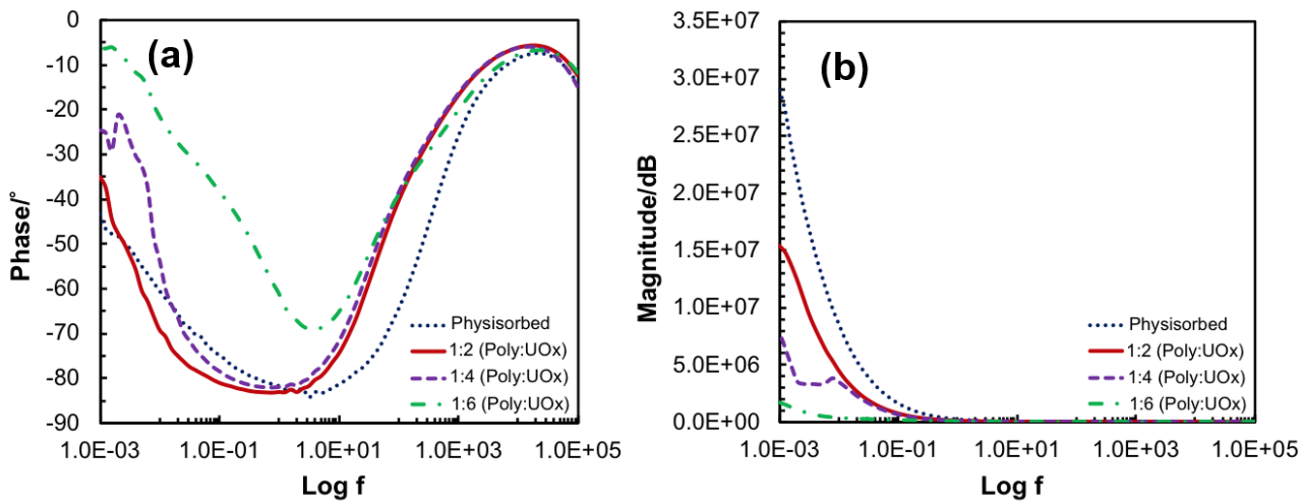

Figure 3.10 Bode plot showing variation in impedance (a) phase angle and (b) magnitude on different UOx loaded substrates with frequency from $0.001 \mathrm{~Hz}$ to 100 $\mathrm{kHz}$ at $0.3 \mathrm{~V}[236]$

increase in frequency. Negative phase angle denoted a lag in response, confirming formation of double layer capacitance. Electrode polarization impedance of the layer formed on the electrode is noted from the slope of the magnitude of double layer capacitance, $C_{\mathrm{dl}}$ at lower frequencies. $C_{\mathrm{dl}}$ formation conforms with conjugation and binding of the UOx to the electrode surface, with accumulation of charge in the double layer.[183] With increased loading of UOx, this capacitive behavior indicates insulating properties on the surface, limiting charge transfer processes. Entrapped enzymatic electrodes have lower $R_{\mathrm{ct}}$ values than the physisorbed due to the ionic interactions between the polymer and $\mathrm{UOx}$ as discussed before. With increased polymer-enzyme ratio, the potential agitation also increased $C_{\mathrm{dl}}$. Simulation and fitting of the data was performed using EIS Spectrum Analyser software. The Nyquist data obtained from EIS was fitted to an equivalent circuit (inset of Figure 3.9a) to determine the $R_{\mathrm{ct}}$ and $C_{\mathrm{dl}}$ at the UOx - FCA interface of the electrodes. The basic Randles circuit was modified by a secondary capacitive element, adapted by a constant phase element (CPE). Due to coulombic interactions and constant motion of FCA molecules towards and away from the electrode, constant diffusion of 
electrons occurs. The diffusion layer, modelled as a CPE, includes capacitive effects at 0.3 $\mathrm{V}$, in parallel with $C_{\mathrm{dl}}$ and $R_{\mathrm{ct}}$.

Surface enzyme activity was studied for each electrode (various polymer-enzyme ratios and physisorbed) to obtain a correlation between the electrochemical behavior of the electrodes and surface activity. For electrochemical studies, the modified electrodes were tested in UA solution $(48 \mu \mathrm{M})$ and the responses were plotted against polymer-enzyme ratios. The enzyme present on the modified electrode surface was quantified by carefully scraping and dissolving the content from the modified electrode surface in a buffer solution and running its absorbance studies at $570 \mathrm{~nm}$. This enzymatic assay was used to calculate the specific activity of UOx on the electrode surface. Compared to physisorbed UOx, the 1:2 ratio polymer entrapped UOx showed $76 \%$ increased activity (Figure 3.9b). However, with further increased ratio of enzyme on the surface, lower activity was noted, with more enzyme retained on the electrode surface using 1:2 ratio. Retaining capability of the enzyme reduced by $60 \%$, ascribed to greater leaching, in higher ratio of functionalization matrix. Electrochemical studies performed from the varied functionalized substrates exhibited higher current density of $\sim 3.42 \mathrm{~mA} \mathrm{~cm}{ }^{-2}$ for $1: 2$ matrix ratio. After immobilization, drying and washing, $92 \%$ enzyme was observed to be retained on the electrode surface in 1:2 ratio $\left(0.23 \mathrm{U} \mathrm{mg}^{-1}\right)$, with more leaching occurring in higher ratios of the functionalization matrix. With vigorous washing of the electrode to remove excess un-entrapped enzyme, increased ratio of enzyme on the electrode surface provided reduced current density, which is consistent with the specific activity results and other characterizations. All these entrapped UOx characterization studies optimized the 
immobilization ratio and suggested evidence for increased presence of active enzyme on the electrode surface using 1:2 matrix ratio.

\subsection{Reaction mechanism in enzymatic Uric Acid detection}

The reaction mechanism of enzymatic UA oxidation is illustrated in Figure 3.11, represented by yellow and green arrows. The enzyme UOx converts UA to 5hydroxyisourate through oxidation (Eq. 2). The formed 5-hydroxyisourate further reacts with water to produce carbon dioxide and allantoin $\left(\mathrm{C}_{4} \mathrm{H}_{6} \mathrm{~N}_{4} \mathrm{O}_{3}\right)$ (Eq. 3). Yield of the byproduct, $\mathrm{H}_{2} \mathrm{O}_{2}$ from this reaction was utilized in well-established colorimetric assay techniques to quantify UA.[88, 184$]$

$$
5 \text { - hydroxyisourate }+\mathrm{H}_{2} \mathrm{O} \rightarrow \text { Allantoin }+\mathrm{CO}_{2}
$$

To monitor the above reaction on the electrode surface, mediated electron transfer (MET) can be applied with the incorporation of a redox electron shuttle. FCA is a redox shuttle known to promote accelerated electron transfer. In widely used oxidase enzymes, FCA receives electrons from the enzyme's co-factor leading to MET, where electron transfer to FCA $\left(E^{0}=-0.4 \mathrm{~V}\right)$ is more favorable.[185-188] In this approach, FCA was used

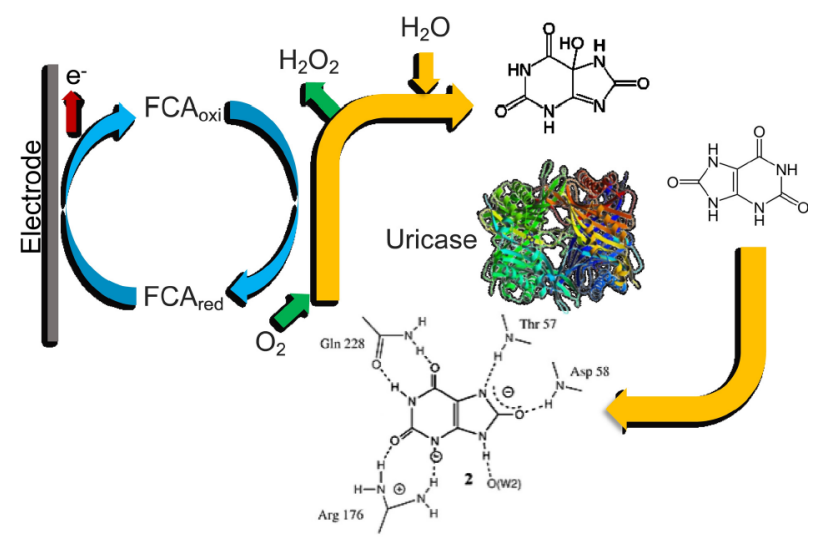

Figure 3.11 Schematic representation of uric acid oxidation by UOx and oxidation of $\mathrm{H}_{2} \mathrm{O}_{2}$ via mediated electron transfer using FCA as redox shuttle [236] 
as an electron transfer mediator to quantify UA oxidation on UOx electrode, as shown in Figure 3.11 and Eq. 4 and 5. The reduced $\mathrm{Fe}^{2+}$ (metal ion in FCA) undergoes a facile oneelectron oxidation on the electrode to the ferricinium state.[189]

$$
\begin{gathered}
\text { Uric Acid }+\mathrm{Fe}^{3+} \rightarrow 5-\text { hydroxyisourate }+\mathrm{Fe}^{2+} \\
\mathrm{Fe}^{2+} \rightarrow \mathrm{Fe}^{3+}+\mathrm{e}^{-}
\end{gathered}
$$

However, UOx does not have any prosthetic group or cofactor; and the $E^{0}$ of UA $\left(E^{0}=0.59 \mathrm{~V}\right)[190]$ being $0.99 \mathrm{~V}$ higher than FCA, electron transfer from the active site of the UA reaction to FCA is thermodynamically not feasible (Eq. 4). Instead, the electron transfer is more favorable toward the native two electron $\mathrm{O}_{2}\left(E^{0}=0.81 \mathrm{~V}\right)$ reduction reaction forming $\mathrm{H}_{2} \mathrm{O}_{2}$ (Eq. 2). To understand the interactions between enzymatic oxidation of UA and FCA, four different experiments were conducted as illustrated in Figure 3.12: (i) behavior of FCA redox couple, (ii) inhibition of $\mathrm{O}_{2}$ in the enzymatic reaction, (iii) inhibition of $\mathrm{H}_{2} \mathrm{O}_{2}$, and (iv) $\mathrm{H}_{2} \mathrm{O}_{2}$ assay studies, to monitor FCA interaction.

(i) Behavior of FCA redox couple: The predominance of $\mathrm{Fe}^{2+}$ ions in the natural state of FCA can be seen in Figure 3.12(a) in the first cycle, where the $I \mathrm{p}_{\mathrm{a}}\left(\mathrm{Fe}^{2+} \rightarrow \mathrm{Fe}^{3+}\right)$ is 1.6 times higher than the second cycle $\left(\mathrm{Fe}^{3+} \rightarrow \mathrm{Fe}^{2+}\right)$ (Eq. 6). However, as seen in the subsequent cycle, the redox ions attained equilibrium $\left(I \mathrm{p}_{\mathrm{a}}=I \mathrm{p}_{\mathrm{c}}\right)$. Hence, both ions are prevalent at the electrode surface.

$$
F e^{2+}-e^{-} \rightleftharpoons F e^{3+}+e^{-}
$$


(ii) Inhibition of $\mathrm{O}_{2}$ in the enzymatic reaction: To understand the role of $\mathrm{O}_{2}$ in electron transfer, our electrode system has been investigated by testing in presence and absence of saturated $\mathrm{O}_{2}$. Results show a significant decrease in $I \mathrm{p}_{\mathrm{a}}$ in the absence of $\mathrm{O}_{2}$ (Figure 3.12(b)). This decrease can be attributed to nil or limited production of $\mathrm{H}_{2} \mathrm{O}_{2}$. In the absence of $\mathrm{H}_{2} \mathrm{O}_{2}$, no electron has been transferred from the active site of the enzyme to the electrode through FCA. This also shows that FCA cannot receive electrons directly from enzymatic UA oxidation (active site). In the absence of $\mathrm{O}_{2}$, some response has been observed due to difficulties in eliminating $\mathrm{O}_{2}$ completely from the saturated solution.
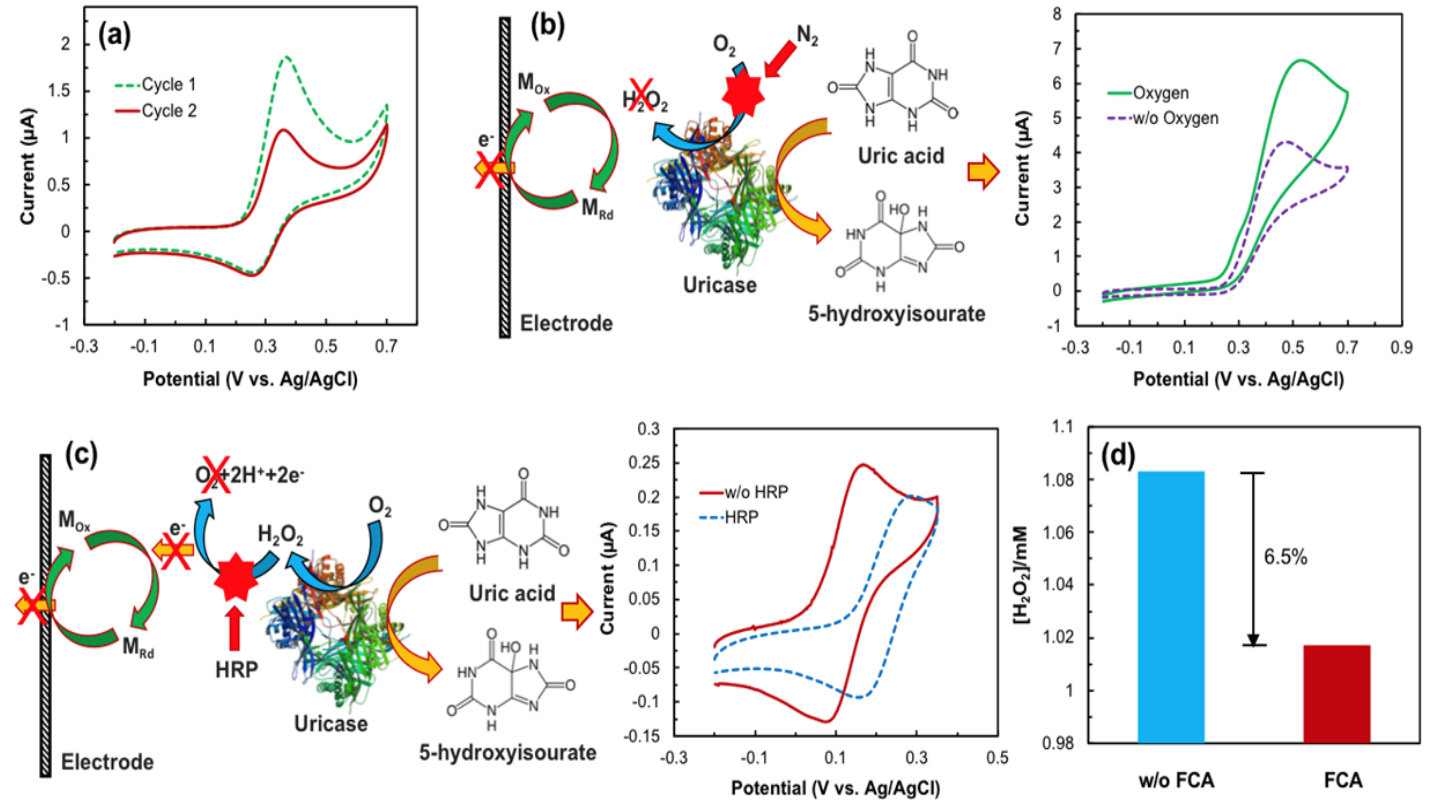

Figure 3.12 (a) CV response of UOx entrapped electrode in presence of $20 \mathrm{mM}$ FCA, showing Cycles 1 and 2 with oxidation peak potential, $E \mathrm{p}_{\mathrm{a}}=0.4 \mathrm{~V}$, (b) Reaction mechanism depicting inhibition of $\mathrm{O}_{2}$ in its absence and presence; and its effect on FCA signal $\left(E \mathrm{p}_{\mathrm{a}}=0.45 \mathrm{~V}\right)$, (c) Electron transfer mechanism depicting inhibition of $1 \mathrm{mM}$ $\mathrm{H}_{2} \mathrm{O}_{2}$ in the absence and presence of HRP; and its effect on FCA signal, (d) Concentration of $\mathrm{H}_{2} \mathrm{O}_{2}$ in presence and absence FCA, measured by $\mathrm{H}_{2} \mathrm{O}_{2}$ assay. All the above $\mathrm{CV}$ measurements were carried out at $20 \mathrm{mV} \mathrm{s}^{-1}$ [236] 
(iii) Inhibition of $\mathrm{H}_{2} \mathrm{O}_{2}$ : The importance of $\mathrm{H}_{2} \mathrm{O}_{2}$ in the electron transfer mechanism has been investigated by consuming the formed $\mathrm{H}_{2} \mathrm{O}_{2}$ with the use of HRP and blocking its reaction with FCA. Results from Figure 3.12(c) show a decrease in $I \mathrm{p}_{\mathrm{a}}$ in the presence of HRP as compared to its absence. This also shows that electron transfer occurs through its by-product $\mathrm{H}_{2} \mathrm{O}_{2}$ to FCA and the electrode surface, the possibility of which has also been reported previously.[168-169, 171] The interaction between the formed $\mathrm{H}_{2} \mathrm{O}_{2}$ and FCA is seen in Figure 3.13, where there is a decrease in $E \mathrm{p}_{\mathrm{a}}$ by $150 \mathrm{mV}$ and an increase in $I \mathrm{p}_{\mathrm{a}}$ by $60 \%$ (at $E \mathrm{p}_{\mathrm{a}} 0.38 \mathrm{~V}$ ) in presence of both FCA as well as $\mathrm{H}_{2} \mathrm{O}_{2}$. This indicates that $\mathrm{H}_{2} \mathrm{O}_{2}$

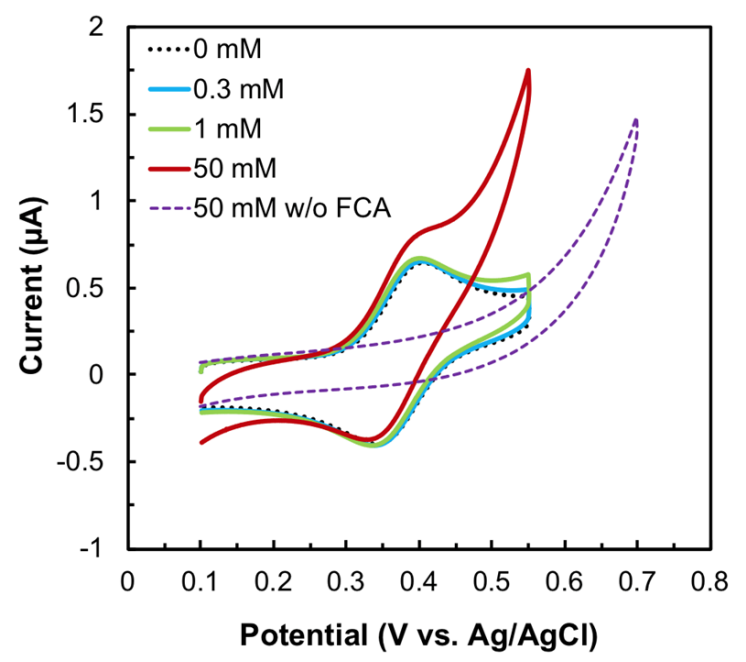

Figure $3.13 \mathrm{CV}$ response of UOx entrapped electrode in presence and absence of 20 mM FCA to $0,0.3,1$, and $50 \mathrm{mM} \mathrm{H}_{2} \mathrm{O}_{2}$ at a scan rate of $20 \mathrm{mV} \mathrm{s}^{-1}$. This reveals that FCA acts as an electrochemical mediator for $\mathrm{H}_{2} \mathrm{O}_{2}$ oxidation [236]

$\left(E^{0}=-0.68 \mathrm{~V}\right)$ acts as a reducing agent in the reaction, with plausible electron transfer to FCA $\left(E^{0}=-0.4 \mathrm{~V}\right)$. On the same electrode (Figure 3.13), in the absence of FCA there was no defined electrochemical peak, showing no $\mathrm{H}_{2} \mathrm{O}_{2}$ oxidation in the same potential window.

(iv) $\mathrm{H}_{2} \mathrm{O}_{2}$ assay studies: Sampling of electrolyte solutions from the above reactions was performed to show that $\mathrm{H}_{2} \mathrm{O}_{2}$ was consumed during FCA redox reaction (Figure 3.12(d)). 
These results show that in the presence of FCA, there is a $6.5 \%$ decrease in $\mathrm{H}_{2} \mathrm{O}_{2}$ concentration, while in the absence of $\mathrm{FCA}$, the $\mathrm{H}_{2} \mathrm{O}_{2}$ concentration remained unchanged.

All the above results support that electron transfer from $\mathrm{H}_{2} \mathrm{O}_{2}$ to FCA (Eq. 7) is thermodynamically more favorable than the UA reaction at the active site to FCA (Eq. 4).

$$
\mathrm{Fe}^{3+}+\mathrm{H}_{2} \mathrm{O}_{2} \rightarrow \mathrm{Fe}^{2+}+\mathrm{O}_{2}+2 \mathrm{H}^{+}+2 e^{-}
$$

\subsection{Electrochemical characterization of entrapped enzymatic biosensor}

For FCA oxidation on a bare electrode at a $\mathrm{pH}$ of 7.8, an $E \mathrm{p}_{\mathrm{a}}$ of $0.4 \mathrm{~V}$ and an $I \mathrm{p}_{\mathrm{a}}$ of $2.6 \mathrm{~mA}$ were respectively noted with a peak separation, $\Delta E=30 \mathrm{mV}$ (Figure 3.14a). The formal potential, $E \mathrm{p}_{\mathrm{a}}$ and $\Delta E$ of the other two electrodes (entrapped and physisorbed) were seen to remain unchanged. In presence of UA, increased current density by $35 \mathrm{~mA} \mathrm{~cm} \mathrm{~cm}^{-2}$ was observed from the enzyme entrapped electrode, as compared to the other three. The entrapped active surface coverage for the electrode $(\Gamma)$ was obtained as $5.57 \times 10^{-14} \mathrm{~mol}^{-1}$ $\mathrm{cm}^{2}$ from the below equation, Eq. 8 .

$$
\Gamma=Q / n F A
$$

where; $\mathrm{F}$ is the faradaic constant, $\mathrm{Q}$ is the quantity of charge consumed with an electron transfer of $\mathrm{n}$ over a physical area, A. Compared to the other two electrodes, the enzyme entrapped electrode has a characteristic steady state response in the scan beyond $0.4 \mathrm{~V}$. This increase can be attributed to improved diffusion of the analyte to the electrode's working area through the polymer-enzyme hybrid matrix. The double fold increase in current density of the entrapped electrode compared to physisorbed shows that there was an increase in enzyme ratio within the polymer matrix.[191] It is seen from Figure 3.14a that, response of the sensor in the absence of FCA, provided much lower signal as compared to, in the presence of FCA. This shows that FCA acts as a mediator in the 
reaction, enabling detection at an $E \mathrm{p}_{\mathrm{a}}$ lower by $200 \mathrm{mV}$. In the absence of UOx, the biosensor showed reduced response by $26.811 \%$. A bare electrode in presence of the same concentration of UA showed lower signal by $18.11 \%$. The biosensor was evaluated by testing it with consecutive UA concentrations $(0,12-100 \mu \mathrm{M})$ in the physiologically relevant ranges of sweat and wound fluid. In presence of FCA, results of the entrapped biosensor showed a linear increase in $I \mathrm{p}_{\mathrm{a}}$ having ordinate at $3.44 \mu \mathrm{A}$, with a linear regression of $y=0.0111 x+3.5251 ; R^{2}=0.94523$ and a standard deviation of $0.04 \mu \mathrm{A}$ with respect to the physiological UA concentrations (Figure 3.14a inset).
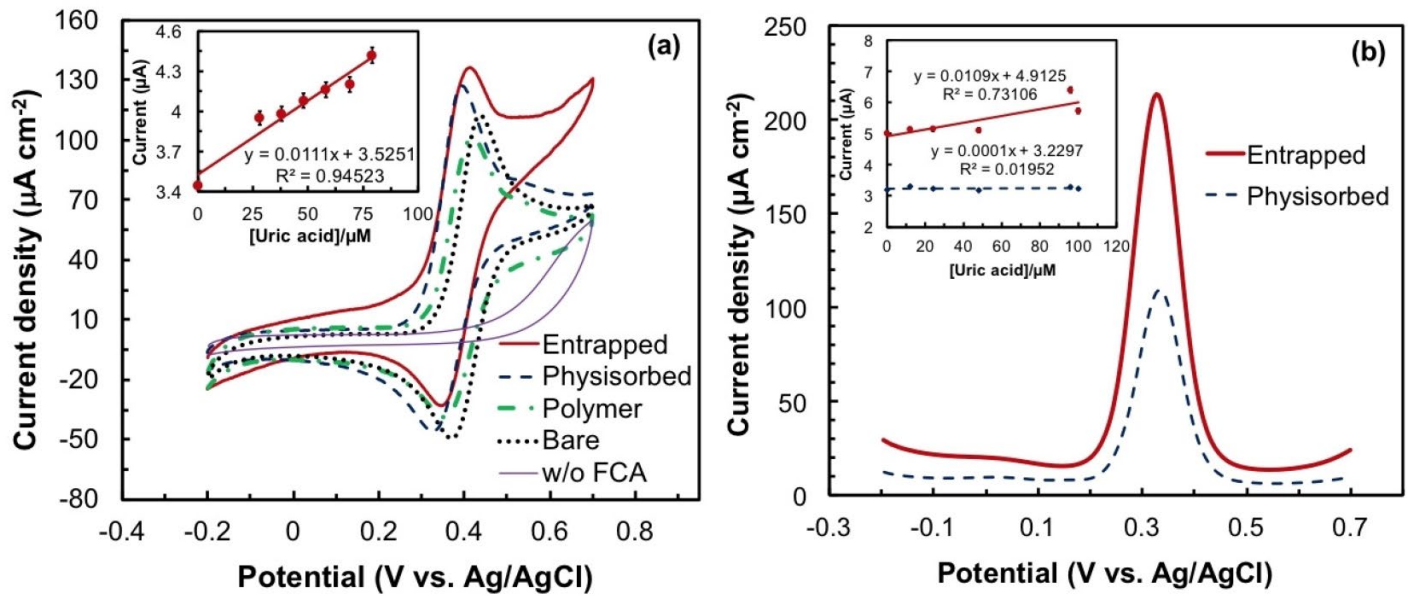

Figure 3.14 (a) $\mathrm{CV}$ response of $48 \mu \mathrm{M}$ uric acid on bare, only polymer, UOx physisorbed and entrapped electrodes in presence and absence of $20 \mathrm{mM}$ FCA at the scan rate of 20 $\mathrm{mV} \mathrm{s}^{-1}$. The inset is the plot of $I \mathrm{p}_{\mathrm{a}}$ Vs. uric acid concentration in physiological range $(12-100 \mu \mathrm{M})$. (b) DPV response of $48 \mu \mathrm{M}$ uric acid on UOx physisorbed and entrapped electrodes at the amplitude $50 \mathrm{mV}$ in presence of $20 \mathrm{mM}$ FCA. In the inset, the continuous line represents entrapped electrode and the dotted line represents physisorbed. [236]

These studies were repeated using the DPV technique as well (Figure 3.14b). Like $\mathrm{CV}$, the $E \mathrm{p}_{\mathrm{a}}$ in DPV is $0.33 \mathrm{~V}$. The obtained current density in DPV was $52.3 \%$ higher than in $\mathrm{CV}$, due to its ability to discriminate against charging (capacitance) currents through pulses, and its sensitivity in yielding peaks to faradaic currents.[191] The UOx 
entrapped electrode provided higher current density compared to a UOx physisorbed electrode as seen from Figure 3.14b. Linear calibration (Figure 3.14b inset) showed that the UOx physisorbed electrode cannot be used for UA detection due to its ultra-low sensitivity value of $100 \mathrm{nA} \mu \mathrm{M}^{-1} \mathrm{~mm}^{-2}$. Sensitivity of the entrapped electrode was 0.155 $\mu \mathrm{A} \mu \mathrm{M}^{-1} \mathrm{~cm}^{-2}$ with an ordinate of $5.01 \mu \mathrm{A}$ from a linear regression of $y=0.01090 x+4.9125$; $R^{2}=0.73106$ and standard deviation of $79.43 \mathrm{nA}$. The UOx entrapped electrode showed superior response, with enzyme activity retained through robust immobilization of the enzyme in its working area, while the physisorbed sensor offered non-linear response with a standard deviation of $32.92 \mathrm{nA}$ on the same scale. With the entrapment technique also providing improved mechanical stability and minimized leaching within its microenvironment, all analyses were performed with enzyme functionalized electrodes, entrapped in the polymeric matrix.

Immobilization of an enzyme affects its catalytic activity. The enzymatic reactions involving a single or multi-substrate with only one varying substrate follow MichaelisMenten kinetics in Eq.9, derived from the Lineweaver-Burk equation,

$$
\frac{1}{I_{S S}}=\frac{K_{\operatorname{mapp}}}{I_{\max }} * \frac{1}{C}+\frac{1}{I_{\max }}
$$


where, $K_{\mathrm{m}(\mathrm{app})}$ is the Michaelis-Menten constant of applied enzyme on the electrode surface, Iss is the steady state current measured at diffusion-controlled region, $I_{\max }$ is the maximum current obtained, and $c$ is the bulk concentration of analyte $\left(48 \times 10^{-9} \mathrm{~mol} \mathrm{~cm}^{-3}\right)$. [192-193] The effective Michaelis-Menten constant for functionalized UOx was determined by electrochemically measuring the diffusion limited current resulting from FCA oxidation $\left(\Delta I \mathrm{p}_{\mathrm{a}}\right) . \Delta I \mathrm{p}_{\mathrm{a}}$ represents the difference in $I \mathrm{p}_{\mathrm{a}}$ between the presence and absence of UA. Assuming the same loading of UOx on both electrodes, the $K_{\mathrm{m}(\mathrm{app})}$ calculated was $1.13 \mathrm{x}$ $10^{-8} \mathrm{M}$. Response of the entrapped electrode in presence and absence of UA is shown in Figure 3.15.

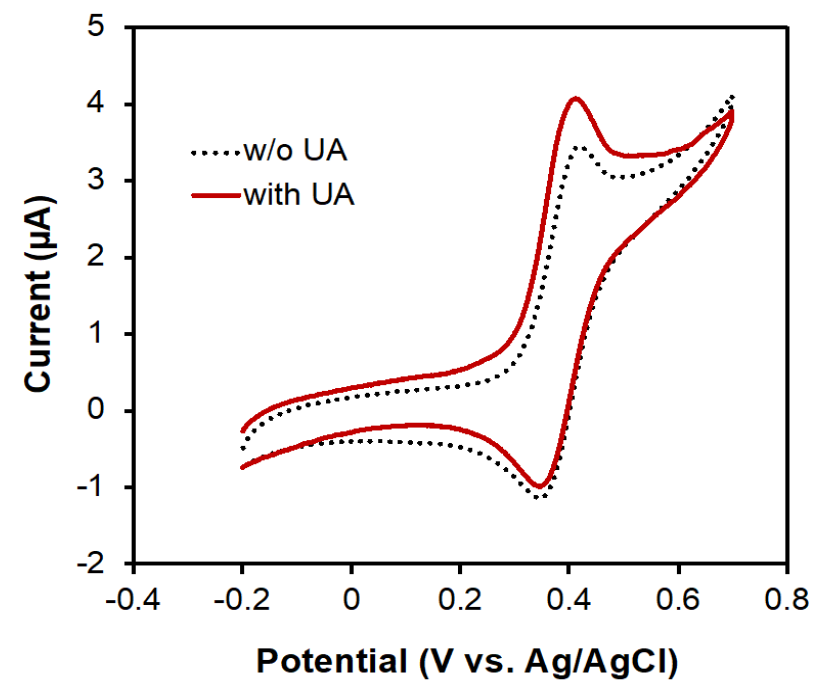

Figure 3.15 CV response of UOx entrapped electrode in presence and absence of UA at a scan rate of $20 \mathrm{mV} \mathrm{s}^{-1}$ [236]

\subsection{Continuous monitoring and stability at physiologically relevant temperatures}

Studies to determine stability of performance during continuous monitoring of UA, were performed over $30 \mathrm{~min}$, in presence of the same concentration of UA $(48 \mu \mathrm{M})$ (Figure 
3.16a). Results showed a gradual decrease in response after $12 \mathrm{~min}$. After 20 min., the prepared biosensor still maintained $80 \%$ of the signal. With each continuous measurement up to $12 \mathrm{~min}$, this biosensor recorded stable response within $10 \%$ variation over 3 days
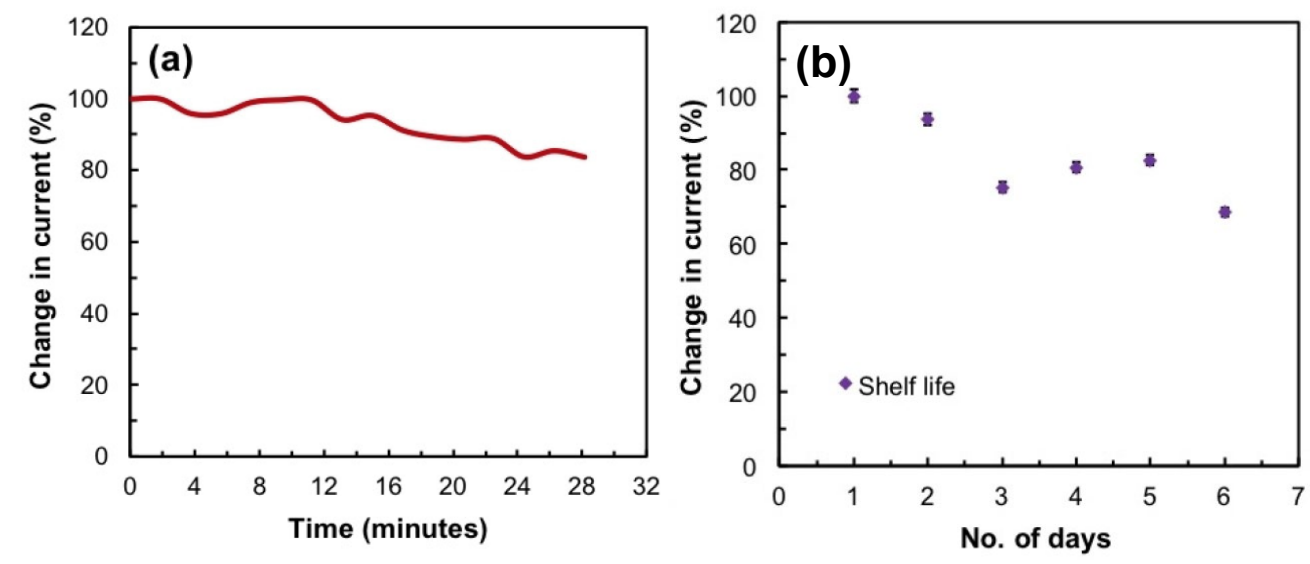

Figure 3.16 (a) Plot representing continuous measurements of the UOx electrode over $30 \mathrm{~min}$ in $48 \mathrm{mM}$ uric acid. (b) Plot representing shelf life of the UOx electrodes, where the measurements were carried out in $48 \mathrm{mM}$ uric acid $[211,236]$

(Figure 3.16a). Stability studies performed over a week in buffer solution containing UA showed that the entrapped sensor offered stable signal over multiple days with repeated use (Figure 3.17a). The physisorbed electrode, however, showed reduced performance by $60 \%$ after day 1.

The enzymatic biosensor was assessed under physiologically relevant variations of temperature to analyze its performance and feasibility for detection from a wound milieu. Measurements were conducted at elevated body temperatures of $40^{\circ} \mathrm{C}$ considering a rise in normal physiological temperature by about $2-3^{0}$ C.[72] Stable performance was observed over a week (Figure 3.17c) in the buffer solution with UA. With each continuous measurement up to $12 \mathrm{~min}$, the biosensor recorded stable response within $\sim 10 \%$ variation 
over 3 days (Figure $3.17 \mathrm{c}$ ). This shows that the entrapped enzymatic biosensor provides stable measurements of UA at physiologically relevant variations of body temperature.
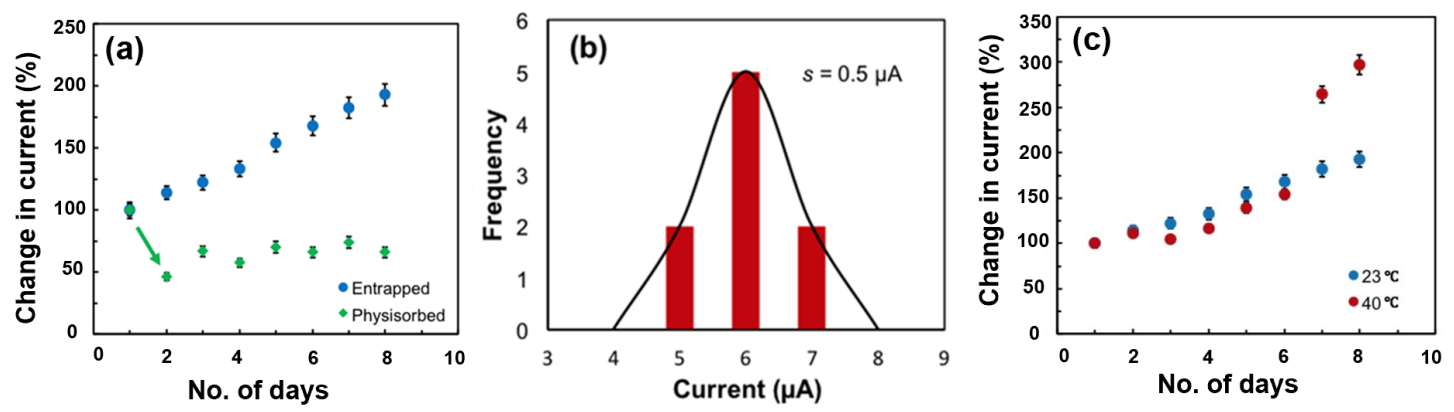

Figure 3.17 (a) Plot representing repeatability of the UOx electrode obtained through repeated measurements of $48 \mu \mathrm{M}$ uric acid over a week; (b) Distribution curve representing reproducibility obtained from nine different UOx electrodes measured at the same condition $[211,236]$

Reproducibility studies were also performed using multiple electrodes. The behavior of these electrodes in the same concentration of UA were plotted to obtain a normal distribution curve (Figure 3.17b). Reproducible results were obtained for a frequency of 5 , providing consistent current of $6 \mu \mathrm{A}$. The distribution data provided a standard deviation of $\sigma=0.5 \mu \mathrm{A}$ for $\mathrm{n}=9$, obtained from Eq. 10 .

$$
\sigma=\sqrt{X}
$$

where, $\mathrm{X}$ is the variance of the current response obtained from the different electrodes. It can be inferred that the obtained standard deviation value provides reasonable response for detection, as can also be seen from the figure (Figure 3.17b). Studies to determine the storage and shelf life of the sensor were performed over a week in buffer solution containing UA. Shelf lives of the enzyme entrapped electrodes were studied in the same concentration of UA over a week's duration. In this experiment, measurements were taken over multiple days and the electrodes were stored at $-4{ }^{0} \mathrm{C}$ when not in use. Results (Figure 3.16b) showed that the electrodes had a shelf life of more than 2 days, after which there 
was a $\sim 20 \%$ decrease in current. The biosensor displayed stable response for continuous measurements with repeated use.

\subsection{Selectivity from major electroactive interferents}

Common electro-active interferents of UA are dopamine (DOP) and ascorbic acid (AA). These analytes are known to have oxidation potentials close to that of UA and demonstrate similar electrochemical behavior. [194] This leads to the close overlapping of response with their oxidation peaks, which are obtained at similar potentials. Healthy human sweat is known to contain $\sim 10 \mu \mathrm{M}(0.18 \mathrm{mg}$. per $100 \mathrm{cc}$.) ascorbic acid (AA).[195] Present in abundance and in much higher concentration in extracellular fluid (46-97 $\mu \mathrm{M})[196]$, it is a key interferent in detection. Known for its biological role as an antioxidant, AA promotes healing through its role in collagen synthesis.[194] Dopamine (DOP), an important neurotransmitter which plays a role in cutaneous wound and dermal tissue healing [197], is also known as an electroactive interferent in UA detection.
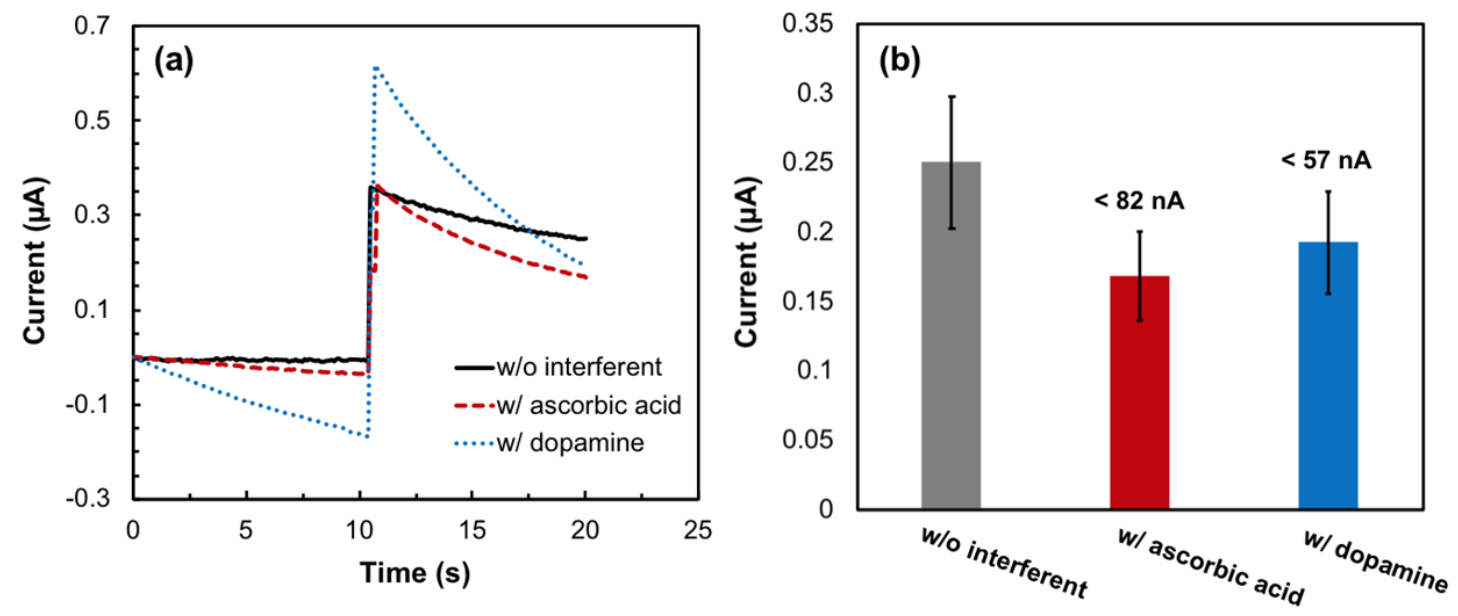

Figure 3.18 (a) Amperometric signal of UA $(100 \mu \mathrm{M})$ in the absence and presence of ascorbic acid $(100 \mu \mathrm{M})$ and dopamine $(100 \mu \mathrm{M})$. (b) Chart representing the reduction in current in presence and absence of ascorbic acid and dopamine at $20 \mathrm{~s}$ [236] 
Amperometric studies have shown (Figure 3.18a) that the biosensor offers response from UA within $5 \mathrm{~s}$. It is observed that in presence of AA, the baseline remains stable and unchanged over $5 \mathrm{~s}$. However, in presence of DOP, there is a shift in the baseline by 0.2 $\mu \mathrm{A}$, after which the signal is noted to stabilize at $10 \mathrm{~s}$. It can also be seen that despite interference in presence of AA and DOP at higher physiological concentrations $(100 \mu \mathrm{M})$, the maximum decrease in current was only $82 \mathrm{nA}$ and $57 \mathrm{nA}(20 \mathrm{~s})$ in presence of DOP (Figure 3.18b). This shows that our biosensor can record reasonable measurements within the physiological range.

\subsection{Conclusion}

Conventional real-time biosensors in wound care are yet to provide direct longitudinal measurements from a wound milieu which can provide a comprehensive understanding of tissue recovery. Although electrochemical investigations have been explored for woundcare, they are mostly non-enzymatic. In this work, an enzymatic biosensor has been designed for continuous, non-invasive detection of wound biomarker, UA. Different characterization techniques were employed to understand the immobilization of UOx within cationic polymer, PVA-SbQ. This unique approach displays superior response as compared to physisorption on a solid support. An optimum ratio of polymer : enzyme was identified to entrap the enzyme; and stable response was obtained with repeated use. Use of FCA as a redox probe provided minimum interference and enhanced response over a wide physiological range through efficient electron transfer between the enzyme and the substrate. This approach has the potential to offer direct measurements from UA, as a biomarker for wound chronicity. 


\section{CHAPTER 4}

\section{NANOCOMPOSITE ENZYMATIC SENSOR FOR MONITORING XANTHINE IN WOUND}

\section{DiAgNOSTICS}

In this study, an enzymatic biosensor employing enzyme XO to monitor Xanthine for potential wound healing assessment has been reported. Mediator free detection, eliminating the use of an external electron shuttle, was investigated through the incorporation of HRP and a specific nanocomposite of multi-walled carbon nanotubes (MWCNT) and gold nanoparticles (AuNP). The role of a nanocomposite of MWCNT/AuNP was explored in enzymatic electrochemical detection of Xanthine for accelerated electron transfer. Inclusion of this nanocomposite was explored to obtain enhanced sensitivity. Sensing response using $\mathrm{CV}$ was recorded within a broad physiological range between $0.1 \mathrm{nM}$ and $7.3 \mathrm{mM}$.

\subsection{Enzymatic detection of Xanthine}

Monitoring the biochemical activity of pertaining analytes aids in leveraging biochemical detection of wound severity. Xanthine is known as an inflammatory biomarker [61] capable of forming reactive oxygen species (ROS) such as hydrogen peroxide $\left(\mathrm{H}_{2} \mathrm{O}_{2}\right)$, which promotes healing. Such species act as scavengers of oxygen radicals to facilitate tissue recovery. [198] In the event of cell rupture, Xanthine is formed in the wound (localized xanthine) from the breakdown of released energy of metabolites in the tissue. Through the utilization of existing cellular biochemical pathways, this work illustrates the viability of a biocompatible electrochemical approach for potential assessment of lesion severity from quantifiable measurements of associated biomarkers. Here, an electrochemical sensor for detection of Xanthine employing enzyme, XO has been 
presented. To facilitate increased reduction of $\mathrm{H}_{2} \mathrm{O}_{2}$ on the electrode, enzyme HRP was introduced in the study. Enzymatic performance of the biosensor was assessed electrochemically, and its sensing performance was recorded to obtain an understanding of the electron transfer between the analyte and the transducer.

\subsection{Effect of physiological pH on detection}

Since physiological events are $\mathrm{pH}-$ dependent, $\mathrm{pH}$ plays a role in the process of lesion healing, influencing the biochemical reactions involved in healing. As wounds are known to shift from a higher to a lower $\mathrm{pH}$ with healing, a correlation could potentially enable calibration of the enzymatic biosensor as a tool for wound monitoring.

Enzyme $\mathrm{XO}$ is known to be active at a slightly alkaline $\mathrm{pH}$ (7.5-7.8), with a pKa value of 6.5 and an isoelectric point around $\mathrm{pH} 6[199-200]$, while HRP is known to provide optimum response around $\mathrm{pH}(6-6.5)$ having a $\mathrm{pKa}$ and isoelectric point of 6.4 and 7.2, respectively.[201-202] To understand the activity of XO/HRP in the mixture and assess electrochemical performance, the bi-enzymatic sensor was analyzed over a $\mathrm{pH}$ range from 5.5 to 9.5. Higher response was obtained between $\mathrm{pH} 7$ and 8 (Figure 4.1), with increased

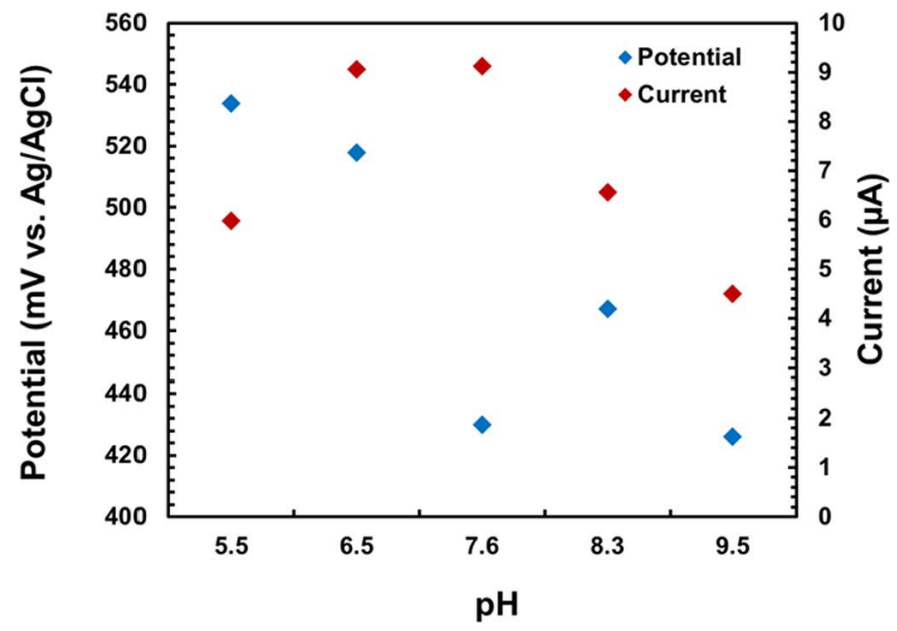

Figure 4.1 Effect of $\mathrm{pH}$ on the current and potential of the bi-enzymatic sensor in presence of xanthine functionalization in presence of Xanthine [244] 
current at $\mathrm{pH} 7.5$ at a lower $E \mathrm{p}_{\mathrm{c}}$. There was a shift in the peak potential with reduced values as the $\mathrm{pH}$ changed from acidic to alkaline.

These investigations offer an improved understanding of the $\mathrm{pH}$ environments suitable for enzymatic detection of Xanthine. This shows that the bi-enzymatic sensor can provide responses in physiologically relevant $\mathrm{pH}$ ranges for wound assessment.

\subsection{Use of nanomaterial in electrochemical detection}

Nanotechnology and different multi-dimensional materials are being extensively explored for the purpose of designing smart, wearable medicinal platforms. Such materials include those of carbon, silver and gold. [76, 203-205] Inclusion of such nanomaterial provides larger electroactive area and higher surface-volume ratios across the electrode surface. Use of suitable material offers increased conductivity across the active area, promoting accelerated electron transfer through increased binding sites and surface interactions. These materials provide augmented response with good electrochemical stability in detection.

\subsubsection{MWCNT/AuNP nanocomposite in detection of Xanthine}

Nanomaterials of carbon and gold notably provide superior performance owing to greater conductivity and catalytic activity.[137, 206-207] Some of their advantages include increased electrode surface area, fast electron transfer, and improved surface confined reactions. Carbon nanotubes (CNT) have open-end structures with edged planes of highly ordered pyrolytic graphite.[208-210] The walls of multi-walled carbon nanotubes (MWCNT) with large basal planes is highly conductive while their edges hold high capacitive and electrocatalytic properties.[210] Studies have shown that its large number of edged planes[208] can enhance molecular redox activity by multiple orders of 
magnitude.[210] Use of AuNP not only improves conductivity but also provides good electrochemical stability in detection. It offers the biosensor with a suitable microenvironment and a biocompatible edge to target wearable healthcare innovation. A combination of MWCNT and AuNP suggests significant improvement in electrochemical detection of Xanthine, to attain enhanced sensitivity and superior response.

\subsection{Experimental}

\subsubsection{Materials}

XO lyophilized powder containing $7 \mathrm{U} \mathrm{mg}^{-1}$ was purchased from Sigma Aldrich and used for Xanthine oxidation. XO enzyme immobilization was performed on SPCE purchased from $\mathrm{CH}$ Instruments, Inc., United States. $0.2 \mathrm{U} \mathrm{ml}^{-1} \mathrm{HRP}$ of analytical grade was used from ThermoFisher Scientific. MWCNT (o.d. $=10-20 \mathrm{~nm}$, i.d. $=2-10 \mathrm{~nm}$ and length $=0.5-200 \mu \mathrm{m}), 20 \mathrm{~nm}$ AuNP $(0.5 \mu \mathrm{M})$, and dimethylformamide (DMF) were purchased from Sigma Aldrich. Xanthine, sodium hydroxide $(\mathrm{NaOH}), \mathrm{H}_{2} \mathrm{O}_{2}$, sodium phosphate monobasic $\left(\mathrm{NaH}_{2} \mathrm{PO}_{4}\right)$ and sodium phosphate dibasic $\left(\mathrm{Na}_{2} \mathrm{HPO}_{4}\right)$ were used of analytical grade. All aqueous solutions were prepared using deionized (DI) water. PBS (pH 7.8) $(20 \mathrm{mM})$ was prepared using $\mathrm{NaH}_{2} \mathrm{PO}_{4}$ and $\mathrm{Na}_{2} \mathrm{HPO}_{4}$ salts and used in electrolyte solutions.

\subsubsection{Apparatus and Methods}

The working electrodes were modified with $20 \mu \mathrm{g} \mathrm{cm}^{-2}$ MWCNT prepared in DMF by drop-casting and drying at $60^{\circ} \mathrm{C}$, followed by $0.5 \mu \mathrm{M}$ AuNP $(1: 1 \mathrm{~V} / \mathrm{V})$ and subsequent drying at $60^{\circ} \mathrm{C}$. Electrode surface characterizations were performed using scanning electron microscopy (SEM JEOL 6330) and transmission electron microscopy (TEM Philips CM200) to understand the surface morphology of the nanocomposite electrodes. Atomic 
force microscopy (AFM) was utilized to obtain an idea of the surface roughness of these functionalized electrodes. Electrochemical impedance studies (EIS) were performed using Metrohm Autolab analyzer to assess the surface impedance characteristics and understand its effect on sensing performance.

The ratio of MWCNT:AuNP functionalization was optimized with 1:1, 1:2, 2:1, 1:3 and 3:1 V/V. The nano-material functionalized electrodes were further modified with enzyme catalysts $0.2 \mathrm{U} \mathrm{ml}^{-1} \mathrm{XO}$ and $0.2 \mathrm{U} \mathrm{ml}^{-1} \mathrm{HRP}$. Mixtures of $\mathrm{XO}$ and $\mathrm{HRP}$ at different molar ratios in PBS $(1: 1,1: 2,2: 1,1: 3,3: 1)$ were explored to immobilize the enzymes onto the MWCNT/AuNP modified electrodes. The amounts of XO and HRP $\left(0.2-0.6 \mathrm{U} \mathrm{ml}^{-1}\right)$ were optimized to obtain improved electron transfer.

All enzyme immobilizations were achieved through drop casting, nitrogen drying, and rinsing in PBS to remove un-entrapped enzymes prior to testing. Drying in nitrogen allowed for the removal of excess water from the electrode surface, forming a gel like structure.[211] All steps involving the enzymes were carried out in an ice-box to reduce denaturation. $\mathrm{CV}$ was performed using the analytical system model $\mathrm{CHI}-230 \mathrm{~B}$ potentiostat from $\mathrm{CH}$ Instruments, Inc. Standard electrochemical characterizations were carried out in a classical three-electrode system consisting of screen printed carbon electrodes (SPCE), an external $\mathrm{Ag} / \mathrm{AgCl}$ as reference electrode, and a Pt counter electrode. This three-electrode system was tested in an electrochemical cell setup with a $3 \mathrm{~mL}$ electrolyte solution to assess the performance of the nanomaterial-enzyme functionalized electrode in different concentrations of Xanthine at a $\mathrm{pH}$ of 7.8. These measurements were performed at $20 \mathrm{mV}$ $\mathrm{s}^{-1}$ to record the generated $\mathrm{H}_{2} \mathrm{O}_{2}$ at the cathode between $-0.1 \mathrm{~V}$ and $-0.6 \mathrm{~V}$. 
Control studies were performed in the absence and presence of different nanomaterials (MWCNT and AuNP) with the working electrode being infused with the enzymes XO and HRP. The electrodes tested were (i) bare, (ii) XO, (iii) AuNP/XO, (iv) MWCNT/XO, (v) MWCNT/Au/XO, (vi) XO/HRP, (vii) AuNP/XO/HRP, (viii) MWCNT/XO/HRP, (ix) MWCNT/AuNP/XO/HRP and (x) MWCNT/AuNP/HRP.

\subsubsection{Electrode functionalization}

It was seen that higher current response was obtained from 1:1 as compared to other ratios (Figure 4.2), owing to uniform distribution of MWCNT:AuNP across the electrode surface. Lower response from increased amounts of AuNP across the MWCNT decorated surface can be attributed to reduced electrode surface area, from coverage of the pores on the carbon substrate.[212] Higher amounts of MWCNT were noted to provide increased capacitance with a small difference in faradaic current.[213] This showed that 1:1 ratio of MWCNT/AuNP offered optimum catalytic response.

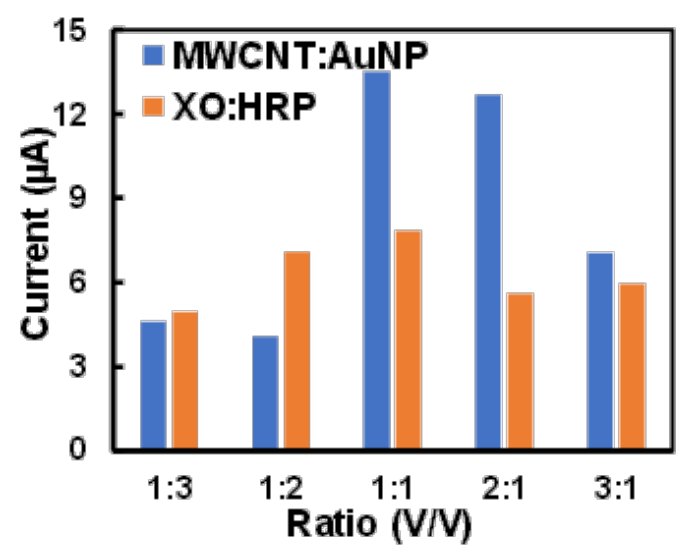

Figure 4.2 Plot depicting change in response with varied MWCNT/AuNP and XO/HRP functionalization in presence of Xanthine [244]

From the different molar ratios of XO:HRP, it was seen that $1: 1$ ratio $\left(0.2 \mathrm{U} \mathrm{ml}^{-1}\right.$ $\mathrm{XO} H \mathrm{HRP}$ ) provided higher signal as compared to the other bi-enzymatic electrodes (Figure 4.2). Reduced current response in higher enzyme ratios can be ascribed to either lower 
production of $\mathrm{H}_{2} \mathrm{O}_{2}$ from decreased amounts of $\mathrm{XO}$, or due to reduced electron transfer from lesser amounts of HRP.[214] This shows that 1:1 ratio of XO:HRP provided optimum measurements.

\subsection{MWCNT/AuNP nanocomposite sensor surface characterization}

SEM Imaging Analysis was utilized to study the surface topography of nanostructure modified electrodes. These studies showed that the MWCNT were randomly distributed across the electrode surface forming a mesh like structure (Figure 4.3a). Such a network of nanostructures allow for improved immobilization of the enzyme with reduced leaching. Deposition of AuNP on these MWCNT decorated electrodes showed a more homogeneous conformation at $200 \mathrm{~nm}$ with the appearance of small globules (Figure 4.3b), spreading across the electrode surface. This comparatively uniform coating of AuNP on the working electrode will offer larger surface-volume ratios to improve the conductivity of the biosensor and increase current signal. [215]
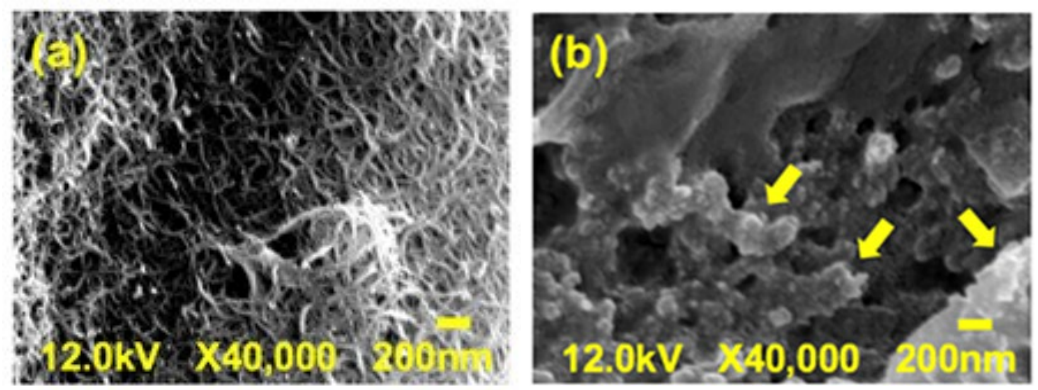

Figure 4.3 SEM images of (a) MWCNT, (b) MWCNT/AuNP decorated electrode [244]

TEM Imaging Analysis showed the network distribution of MWCNT across the sensor surface (Figure 4.4a). Diameter of structures distributed in the MWCNT matrix is about 12 $\mathrm{nm}$, as observed from the high resolution TEM image at $20 \mathrm{~nm}$ (inset: Figure 4.4a). This assisted in providing higher electrochemical stability from increased conductivity of its 

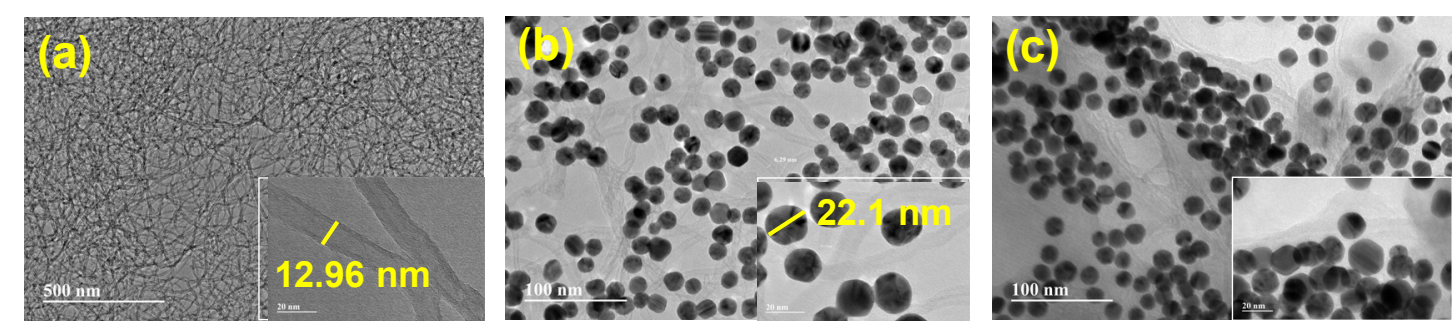

Figure 4.4 TEM images of (a) MWCNT, $\mathrm{MWCNT} / \mathrm{AuNP} / \mathrm{XO}$ decorated electrodes

edged planes and higher electroactive area. Incorporation of 20-22 nm AuNP on the MWCNT decorated surface (inset: Figure 4.4b) offered to further increase the electroactive area with increased conductivity, to promote electron transfer between the transducer and the analyte. Surface interactions were suggested by the deposited AuNP across the MWCNT functionalized surface (Figure 4.4b). Spatial distribution of the AuNP displayed visible agglomeration across specific sites of the MWCNT framework, depicting increased sites for binding. This can augment electron transfer with improved electrocatalytic properties. Incorporation of enzyme onto the nanocomposite functionalized sensor, illustrated its homogeneous integration with the nanocomposite surface, by the formation of a relatively uniform layer across it (Figure $4.4 \mathrm{c}$ ). The enzyme was seen to form a coating over areas of the nanocomposite surface. As seen, this enzyme blanket was noted to conceal the underlying MWCNT mesh in the region. This can be ascribed to increased interactions of the enzyme with the functionalized sensor surface, arising from accredited increase in binding sites offered by the nanocomposite.

AFM imaging analysis provided an understanding of the average surface roughness of the nanocomposite functionalized sensor surface at atomic resolution. At a depth of about 165 $\mathrm{nm}$, the appearance of MWCNT was noted (Figure 4.5a), with the presence of globule clusters denoting the presence of AuNP. Visible agglomeration of AuNP is distinct at a 
(a)

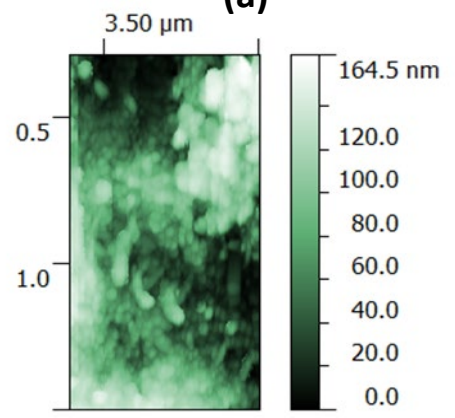

(b)

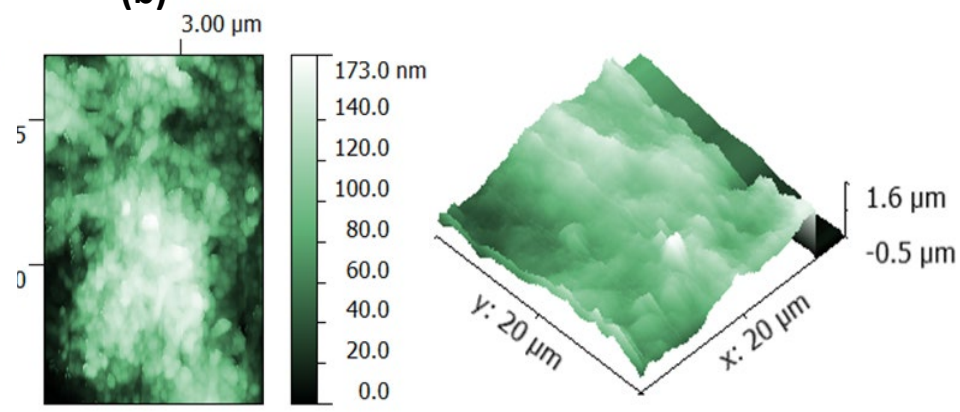

Figure 4.5 AFM images of MWCNT/AuNP nanocomposite electrodes at different depths of (a) $164.5 \mathrm{~nm}$, (b) $173 \mathrm{~nm}$ and (c) $1.6 \mu \mathrm{m}$

depth of $173 \mathrm{~nm}$ (Figure 4.5b). This suggests obvious interactions between the MWCNT and AuNP distributed across the sensor surface. Surface topography of the nanocomposite at a height of $1.6 \mu \mathrm{m}$ was observed (Figure 4.5c). This nanocomposite surface provided an average surface roughness (ASR) of approximately $0.12 \mu \mathrm{m}$.

EIS characterization of the nanocomposite sensor was conducted between $1 \mathrm{mHz}$ and 100 $\mathrm{kHz}$ at a potential of $5 \mathrm{mV}$ in buffer to explore the electron transfer properties. Compared to the bare electrode, a reduction in $R_{\mathrm{ct}}$ was observed from the MWCNT/AuNP

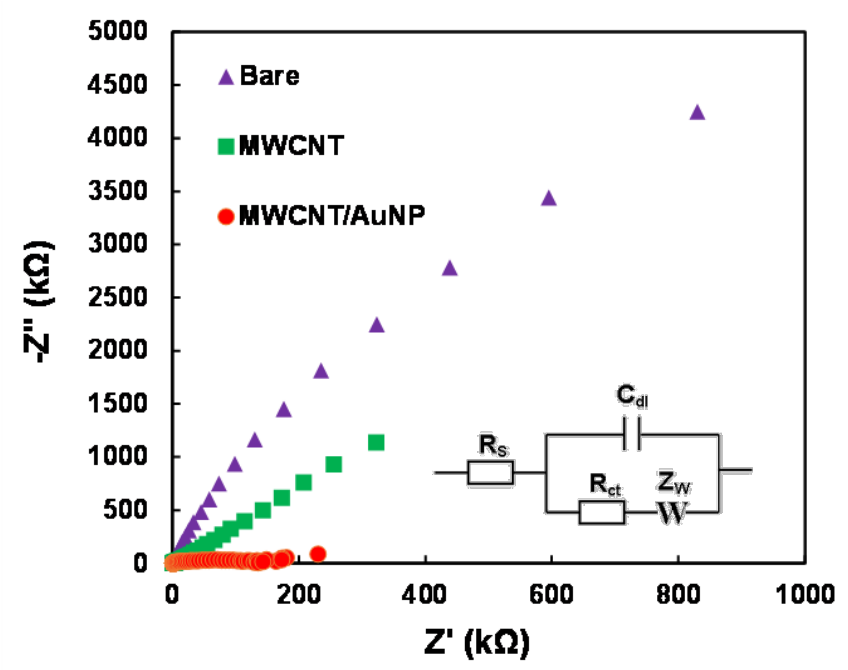

Figure 4.6 Nyquist plot showing variation of impedance on electrodes functionalized with MWCNT and MWCNT/AuNP at $5 \mathrm{mV}$ between $1 \mathrm{mHz}$ and $100 \mathrm{kHz}$ in presence of buffer 
nanocomposite electrode as observed from the Nyquist plot (Figure 4.6). Decrease in electron transfer resistance from $30940 \mathrm{k} \Omega$ to $168.55 \mathrm{k} \Omega$ can be accredited to increased conductivity from the hybrid nanocomposite matrix. A decrease in $R_{\mathrm{ct}}$ by $1512.45 \mathrm{k} \Omega$ was observed with the integration of AuNP on the MWCNT functionalized surface. This denoted increase in conductivity from increased interactions between the MWCNTs and AuNPs, suggesting increased electron transfer properties.

\subsection{Reaction mechanism employed in detection}

Xanthine dehydrogenase is prevalent in normal tissue and transforms to $\mathrm{XO}$ in the case of injury. XO is an oxidoreductase enzyme with a cofactor, FAD that facilitates its oxidation to terminal purine product, UA. XO has 2 molecules of FAD bridged by a pair of ferric mercaptide groups.[216] Purine substrates bound by the isoalloxazine ring system of one FAD undergoes oxidation in presence of $\mathrm{O}_{2}$ and $\mathrm{H}_{2} \mathrm{O}$ (Eq. 1). The presence of a molybdenum cofactor in $\mathrm{XO}$ allows for the transfer of electrons in the oxidation pathway to convert to by-product $\mathrm{H}_{2} \mathrm{O}_{2}$. The oxidation pathway of Xanthine as illustrated in Figure 4.7, was utilized in the study, with the enzymes acting as catalysts to facilitate detection.

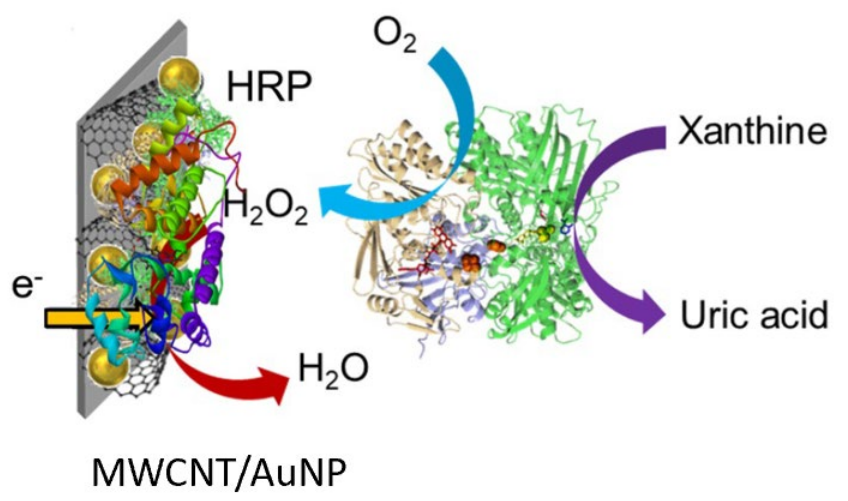

Figure 4.7 Schematic showing electron transfer mechanism in xanthine oxidation by XO and HRP on a nanocomposite functionalized electrode [244] 
Enzymatic oxidation of Xanthine was quantified by measuring the formed $\mathrm{H}_{2} \mathrm{O}_{2}$ on the electrode.

HRP, being a metalloenzyme, has an iron heme group and contains a histidine residue, which is in a vacant resting site.[217] $\mathrm{H}_{2} \mathrm{O}_{2}$ can attach to this vacant site during its reduction-oxidation reactions, where an oxygen atom can bind during activation. This position is considered as the active site for enzyme binding.[217] It can be observed from Eq. 11 that $\mathrm{HRP}$ catalyzes the reduction of $\mathrm{H}_{2} \mathrm{O}_{2}$ to water and oxygen.

$$
\text { Horseradish peroxidase }+\mathrm{H}_{2} \mathrm{O}_{2} \rightarrow \mathrm{H}_{2} \mathrm{O}+\mathrm{O}_{2}
$$

Presence of HRP was noted to improve electron transport of $\mathrm{H}_{2} \mathrm{O}_{2}$ by enabling rapid transfer of electrons between the enzyme and the transducer. This enabled increased reduction of $\mathrm{H}_{2} \mathrm{O}_{2}$, in turn offering amplified signal output with improved quantification for biomarker detection.[218-219]

The use of physiologically relevant enzymes allows them to act as natural catalysts in detection. Utilizing such enzymes allows for improved electrocatalytic activity with specific response to the target analyte.

\subsection{Role of MWCNT/AuNP nanocomposite in Xanthine detection}

Potentiometric analyses enabled assessment of electrochemical performance of the nanomaterial-enzyme functionalized electrode in a broad range between $0.1 \mathrm{nM}$ and 7.3 $\mathrm{mM}$. Studies were conducted with different increasing concentrations of Xanthine prepared in PBS at $\mathrm{pH}$ 7.5. These measurements were performed at $20 \mathrm{mV} \mathrm{s}^{-1}$ to record the generated $\mathrm{H}_{2} \mathrm{O}_{2}$ at the cathode between $-0.1 \mathrm{~V}$ and $-0.6 \mathrm{~V}$, with all potentials reported with respect to $\mathrm{Ag} / \mathrm{AgCl}$ reference electrode. 
In this process, the $\mathrm{H}_{2} \mathrm{O}_{2}$ byproduct reduction (Eq. 12) was monitored on the MWCNT/AuNP/XO functionalized substrate, to measure the electrical signal generated from the exchange of electrons between the electrode and the reduction reaction.

$$
\mathrm{H}_{2} \mathrm{O}_{2}+2 \mathrm{H}^{+}+2 e^{-} \rightarrow 2 \mathrm{H}_{2} \mathrm{O}
$$

Reduction signal for the enzymatic nanocomposite sensor was noted to have a lower onset voltage $(0.2 \mathrm{~V})$ with a higher slope compared to XO or only MWCNT, AuNP or bare electrode (Figure 4.8a) revealing its enhanced electrocatalytic properties. In presence of Xanthine, the nanocomposite has a peak at $E \mathrm{p}_{\mathrm{c}}-0.5 \mathrm{~V}$, however no definite peak appeared for the other three electrodes. It can be seen from the same figure that a MWCNT/XO functionalized electrode provided higher signal for Xanthine by $15 \mu \mathrm{A}$, owing to greater electroactive area as compared to AuNP/XO modified electrode. A combination of both the MWCNT and AuNP, however, was seen to provide significant increase in response as compared to their use individually. At an $E \mathrm{p}_{\mathrm{c}}$ of $-0.5 \mathrm{~V}$, in the same concentration of Xanthine, $I \mathrm{p}_{\mathrm{c}}$ of $36.4 \mu \mathrm{A}$ was observed from a MWCNT/AuNP/XO
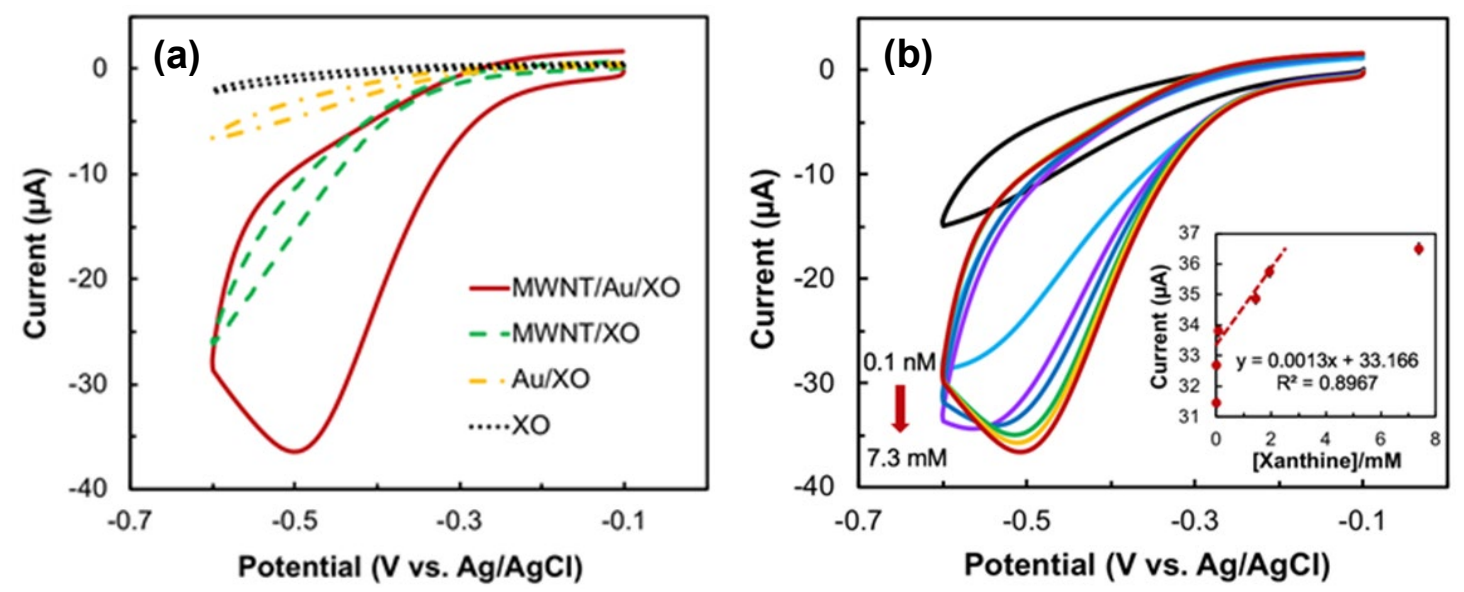

Figure 4.8 (a) Comparison of $\mathrm{CV}$ signals of various $\mathrm{XO}$ electrodes in presence of xanthine, (b) CV signals of various xanthine concentrations from MWCNT/AuNP/XO biosensor. Inset: Linear regression plot of the biosensor [241] 
modified electrode. This nanocomposite electrode offered a 36-fold increase in response as compared to other enzymatic electrodes.

The nanocomposite modified biosensor was evaluated by testing it with different increasing concentrations of Xanthine between $0.1 \mathrm{nM}$ and $7.3 \mathrm{mM}$. As depicted in Figure $4.8 \mathrm{~b}$, a linear increase in $I \mathrm{p}_{\mathrm{c}}$ was observed from $11.2 \mu \mathrm{A}$ to $36.5 \mu \mathrm{A}$, with increase in concentration. This nanocomposite modified XO electrode offered a sensitivity of 18.57 $\mathrm{nA} \mu \mathrm{M}^{-1} \mathrm{~cm}^{-2}$ with enzyme activity retained through irreversible immobilization of the enzyme on its working area. The enzyme immobilization processes provided improved performance by $25 \mu \mathrm{A}$ with minimized leaching within its microenvironment, allowing diffusion of the analyte towards the working area of the transducer surface.

\subsection{Bi-enzymatic performance using MWCNT/AuNP nanocomposite}

To enable enhanced electron transfer between the electrode and the analyte, HRP enzyme was introduced in the system to investigate Xanthine detection. The presence of HRP increased the current density by $71.42 \mu \mathrm{A} \mathrm{cm}^{-2}$ at an $E \mathrm{p}_{\mathrm{c}}=0.5 \mathrm{~V}$ in presence of Xanthine. This electrode also displayed lower onset potential ( $0.2 \mathrm{~V})$ compared to a single enzymatic electrode. This indicates improved electrocatalytic activity. In comparison, a bare electrode shows no definite peak due to no formed $\mathrm{H}_{2} \mathrm{O}_{2}$. The bi-enzymatic electrode was thus seen to show an enhanced response to the same concentration of Xanthine. 
Electrochemical analyses showed that XO provided an onset potential of $-0.3 \mathrm{~V}$ in presence of Xanthine with no defined reduction peak (Figure 4.9a). This was due to sluggish reduction in the XO functionalized substrate. The presence of isolated MWCNT or Au nanomaterial on the bi-enzymatic electrode provided lower response in presence of Xanthine (Figure 4.9b). A MWCNT functionalized enzymatic electrode provided a 2-fold increase in signal as compared to AuNP modified electrode. The presence of a nanocomposite from a combination of MWCNT and AuNP offered a further enhanced response from the enzymatic electrode at a low onset potential of $0.3 \mathrm{~V}$. An increase in current signal by $7 \mu \mathrm{A}$ was observed at an $E \mathrm{p}_{\mathrm{c}}=0.5 \mathrm{~V}$ with a lower onset potential of 0.2 V.
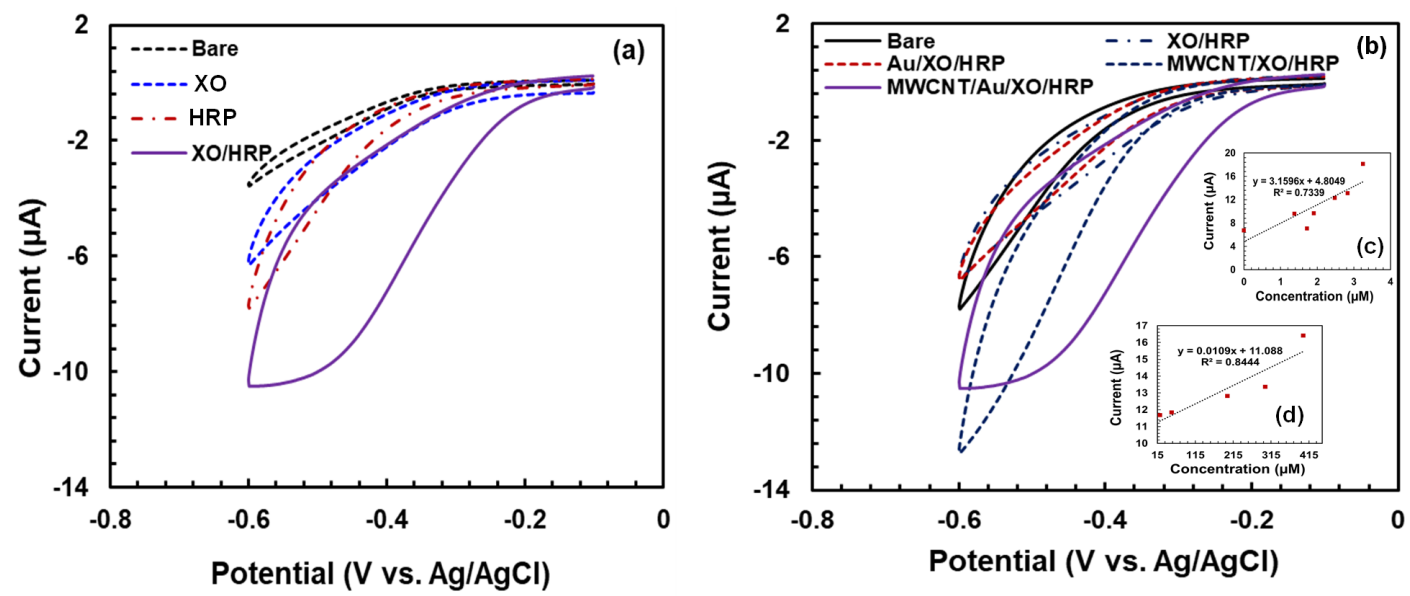

Figure 4.9 (a) CV signals of bare, MWCNT/Au functionalized with XO or HRP or both in presence of $32 \mu \mathrm{M}$ xanthine, (b) $\mathrm{CV}$ signals of bare, XO/HRP electrodes with MWCNT or Au or MWCNT/Au in presence of $32 \mu \mathrm{M}$ xanthine; Insets: (c) Linear regression plot of the bi-enzymatic sensor, (d) Linear regression plot of the sensor at elevated concentrations [244]

Electrochemical response from the nanocomposite electrode was evaluated in the presence of increasing concentrations of Xanthine in the physiologically relevant ranges for wound monitoring. Typical concentrations of Xanthine are known to vary between 3 and $5 \mu \mathrm{M}$ in healthy individuals with therapeutic levels rising from 35 to $160 \mu \mathrm{M} .[63,220]$ 
Sensor response employing the nanocomposite of MWCNT/AuNP was evaluated within 6 $\mu \mathrm{M}$ (inset: Figure 4.9c) and elevated concentrations (inset: Figure 4.9d). Sensor design employing this nanocomposite assisted in providing a linear response between $22 \mu \mathrm{M}$ and $0.4 \mathrm{mM}$ (inset: Figure 4.9d), broader than the physiological range. With a detection limit of $1.3 \mu \mathrm{M}$, enhanced performance was recorded (inset: Figure $4.9 \mathrm{c}$ ). An increase in $I \mathrm{p}_{\mathrm{c}}$ was recorded when subjected to different increasing concentrations of Xanthine within the physiologically relevant range. This nanocomposite modified bi-enzymatic electrode offered a sensitivity of $155.71 \mathrm{nA} \mu \mathrm{M}^{-1} \mathrm{~cm}^{-2}$ through irreversible immobilization of the enzyme on the working area. Improved response was obtained in the presence of increased concentrations of Xanthine due to increased reduction of $\mathrm{H}_{2} \mathrm{O}_{2}$ on the nanocomposite functionalized bi-enzymatic sensor.

The nanocomposite of MWCNT/AuNP offered larger electroactive area for improved enzyme loading and enhanced sensitivity. This uniformly spread nanomaterial composite provided greater electrocatalytic activity ratios.[221] This approach incorporating enzymes, $\mathrm{XO}$ and $\mathrm{HRP}$, in combination with the nanocomposite of MWCNT/AuNP, allowed for improved quantification in Xanthine biomarker detection.[5, 222]

\subsection{Interference studies}

Wound exudate has high protein content, comprising several electrolytes and inflammatory components.[223] Analogy of acute wound fluid has previously shown the presence of different ions like sodium, potassium, chloride, urea and creatinine; besides other cytokines, leucocytes, lysozymes, macrophages, neutrophils and other microorganisms. Thus, biochemical analysis of a wound environment using an electrochemical 
biosensor may be affected by interference from such electroactive species. Among the wound fluid components, such species are hypoxanthine, uric acid, ascorbic acid and dopamine. Xanthine is produced from hypoxanthine and leads to the formation of uric acid in purine metabolism, using $\mathrm{XO}$.

$\mathrm{XO}$ being the key catalyst in the biosensor, such purine metabolites are interferents in detection. Like purine metabolites, the wide presence of electroactive species such as ascorbic acid and dopamine in humans will also interfere with Xanthine detection. Under physiological conditions, interference from hypoxanthine $(8 \mu \mathrm{M})$ and uric acid $(0.5 \mathrm{mM})$ were thus investigated, in the same potential window. It can be seen from Table 4.1, that change in response between $3.8 \%$ and $9.9 \%$ was observed with the presence of these interferents. With the presence of ascorbic acid $(34 \mu \mathrm{M})$, comparatively higher interference was noted, with a change in response by $11.8 \%$, while dopamine (196 pM) was seen to depict an interference of $8.8 \%$. Interferent concentrations were chosen as per the higher biological levels, with detection in presence of lower physiological concentrations of Xanthine. This shows that even in presence of higher concentrations of the common electroactive interferents, the sensor signal for lowest concentration of Xanthine does not deviate beyond (+/-) $6 \%$. Thus, this enzymatic biosensor can be utilized to determine Xanthine in physiologically relevant biofluids.

\begin{tabular}{ccccc}
\hline $\begin{array}{c}\text { Interfering } \\
\text { biomolecules }\end{array}$ & $\begin{array}{c}\text { Interferent concentration } \\
(\mu \mathrm{M})\end{array}$ & $\begin{array}{c}\text { Response without } \\
\text { interferent }(\mu \mathrm{A})\end{array}$ & $\begin{array}{c}\text { Response with } \\
\text { interferent }(\mu \mathrm{A})\end{array}$ & $\begin{array}{c}\text { Change in } \\
\text { response }(\%)\end{array}$ \\
\hline Uric Acid & 500 & 12.05 & 13.25 & 9.96 \\
Hypoxanthine & 8 & 11.06 & 11.49 & 3.89 \\
Ascorbic Acid & 34 & 9.14 & 10.23 & 11.93 \\
Dopamine & 0.000196 & 15.51 & 16.88 & 8.85 \\
\hline
\end{tabular}

Table 4.1. Response of sensor for $3 \mu \mathrm{M}$ xanthine in absence and presence of interfering biomolecules [244] 


\subsection{Conclusion}

A bi-enzymatic biosensor was developed using two enzymes, XO and HRP, for detection of Xanthine within physiologically relevant ranges. Electrochemical analyses from nanomaterial modified bi-enzymatic electrodes provided enhanced responses with an increased reduction of by-product $\mathrm{H}_{2} \mathrm{O}_{2}$. Linear response was obtained with increasing concentrations of Xanthine over the concerned range. Incorporation of specific MWCNT/AuNP nano-composite in this study paves a path for improved detection of such purine metabolites, with a potential for quantitative assessment of healing. 


\section{CHAPTER 5}

\section{DESIGN OF BIOSENSOR FOR WEARABLE WOUND MONITORING AND CLINICAL SAMPLE}

\section{VALIDATION}

In this chapter, specific properties of materials employed in sensor design have been investigated. Hydrophilicity of the sensor surface was explored using Corona Plasma, to obtain an understanding of increased loading and functionalization across the substrate. Electrochemical performance of screen-printed-carbon based electrodes fabricated on cotton gauze was evaluated in enzymatic UA detection for wearable wound monitoring. For the first time, analyte measurements from clinical extracts of purulent samples were recorded using optical and electrochemical techniques, to establish non-invasive detection of UA and Xanthine in relevant biological fluids from around injured tissue.

\subsection{Sensor surface hydrophilicity}

Materials extensively explored in attaining enhanced electrochemical response include nanomaterials of MWCNT and AuNP. Although they provide improved conductivity and electrocatalytic performance with good electrochemical stability, these materials impart increased hydrophobicity to the surface of the transducer, owing to the presence of nonpolar groups. [224-225] This restricts increased loading and functionalization across the transducer surface, attenuating response from it. The use of Corona Plasma was investigated in this study, to improve the hydrophilic properties of the functionalized sensor surface. A hydrophilic surface, with the presence of polar groups, offers improved adhesion and wettability to confine surface molecules onto the desired area for augmented response.

Corona Plasma is a low temperature surface treatment performed at atmospheric pressure. It is a technique which enables polarization of a surface through activation of the 
surface chemistry of its particles. In this method, a high voltage is applied between the tip of a sharp, pointed electrode which has the ability to ionize air in a limited space around it, and a counter-electrode connected to ground. A schematic of this setup is shown in Figure 5.1a.
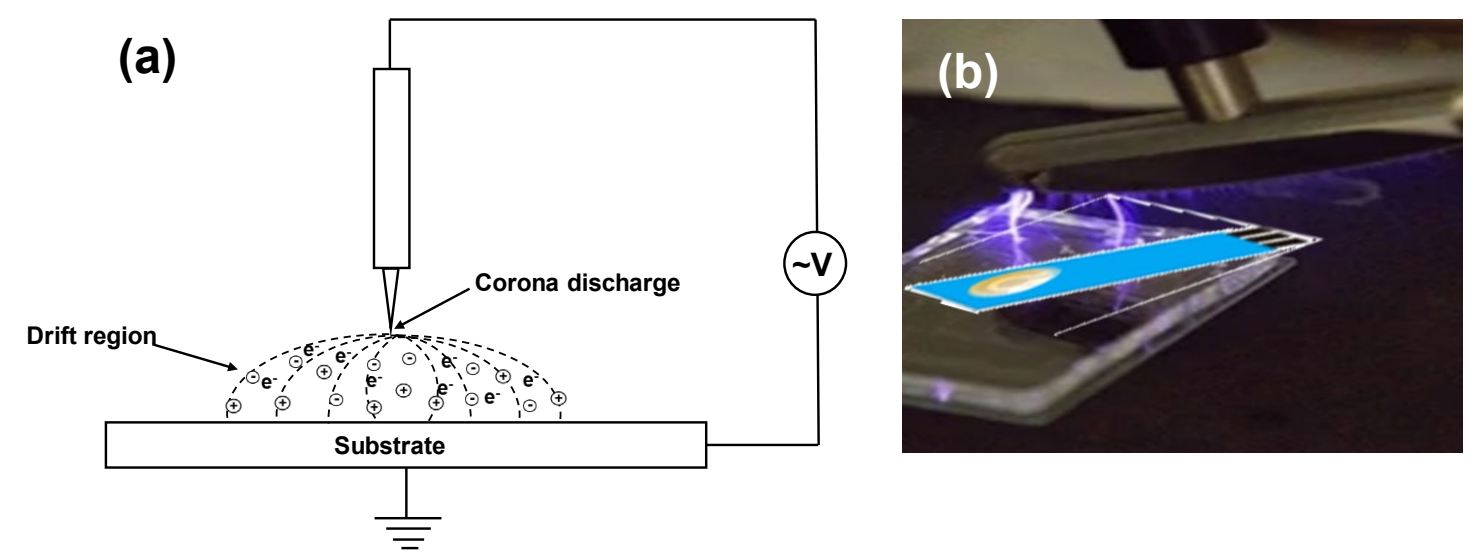

Figure 5.1 (a) Schematic displaying the working mechanism of Corona Plasma in air, (b) Experimental setup of electrode treatment using Corona Plasma

Increased acceleration of electrons in the field generated near the tip, creates an electron avalanche which increases ionization of the surrounding air molecules. At a point where this non-uniform electric field in air becomes almost negligible at a certain distance from the electrode, a potential gradient develops when a corona glow discharge is ignited. This electric discharge causes increased ionization, creating dielectric breakage of air from increased collision of atoms, electrons and ions. The produced corona energy breaks molecular bonds of the surface to which it is directed, increasing the surface energy of the substrate. Open molecular chains bond with free oxygen radicals forming additional polar groups on the substrate surface, which have strong affinity to polar solvents. The formed radicals react with the corona discharge in air to create polar functional groups and impart them to the directed surface, thereby increasing its hydrophilicity and improving its adhesion and wettability characteristics. Such treatment possesses the ability to selectively 
modify non-polar surfaces by modifying its surface tension. Once the energy of the surface molecules exceeds the surface tension of the solvent molecules, adsorption/wettability takes place when the adhesive forces between the substrate surface and the liquid exceed the cohesive forces of surface tension between the liquid molecules on the surface.

Intensity of the electric field generated at the tip activates the surface energy of its particles. Surface activation from electric discharge in limited air space improves adhesion properties of the surface thereby enhancing its bonding properties, preventing detachment of coatings from the surface. Improved adhesion of chemicals and polymers is obtained from enhanced physical and dipolar interactions, through the introduction of reactive groups and free radicals to the surface, in such treatment. This allows for increased loading and functionalization to enable improved response.

When treating a carbon functionalized surface, the chemical interactions between oxygen molecules in air and the substrate surface increases with the creation of strong covalent bonds of greater polarity. With surface modifications from deposited polar functional groups, increased surface tension results from increased energy of the surface particles. While altering the surface chemistry of its particles, adsorption of reactive groups occur on the surface, imparting changes to surface conductivity and electroactive area. Increased conductivity results from the generated positive ions from increased ionization, that are drawn to the negative ions on the substrate surface, in an effort to neutralize to atoms.

\subsection{Detection of wound biomarker from wearable construct}

Wearable biosensing for wound management offers promise in miniaturized, economical and convenient therapy through smart technology. As an attempt to transform 
wound care with wearable monitoring of healing, the design feasibility of a biosensor on a flexible, healing construct has been evaluated in this study. The designed biosensor aims toward personalized wound monitoring, complementing wearable wound management in medical IoT. [211]

Design of an electrochemical biosensor was examined on a flexible, healing construct of cotton gauze. A three-electrode sensing system was fabricated, comprising enzyme UOx entrapped in PVA-SbQ on the working electrode, using $\mathrm{Ag} / \mathrm{AgCl}$ as reference electrode. $\mathrm{A}$ miniaturized, flexible, electronics platform with an ultra-low power potentiostat and microcontroller were built and tested for wireless data transfer to assess the feasibility of the enzymatic sensor for detection of biomarkers from extracts of biofluids in and around an injury.

\subsection{Non-invasive detection of wound biomarkers}

To evaluate this biosensor for human subject analyses, we investigated detection of UA and Xanthine in extracted samples of biofluid from sweat and wound milieu. When an injury occurs, fluid oozes in and around a wound through diffusion in the sweat glands and surrounding tissue in the dermal layers of skin with changes in osmotic gradients (Figure

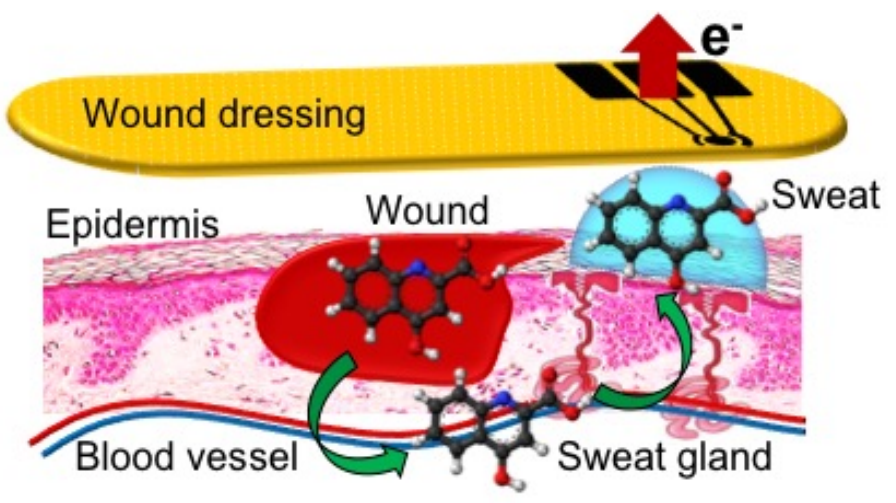

Figure 5.2 Schematic displaying diffusion of analyte from sweat glands through underlying dermal and epidermal layers of skin 
5.2). At the site of an injury, its vicinity also has a rise in analyte concentrations. With UA concentrations between of 20 and $30 \mu \mathrm{M}$ in sweat [226], measuring this analyte noninvasively from sweat in wound proximity is relatively easy and possible.

\subsection{Experimental}

\subsubsection{Materials}

UOx lyophilized powder containing $15 \sim 30 \mathrm{U} \mathrm{mg}^{-1}$ and XO lyophilized powder containing $7 \mathrm{U} \mathrm{mg}^{-1}$ were purchased from Sigma Aldrich, for UA and Xanthine oxidation respectively. UA, Xanthine, sodium hydroxide, $\mathrm{H}_{2} \mathrm{O}_{2}$, sodium phosphate monobasic $\left(\mathrm{NaH}_{2} \mathrm{PO}_{4}\right)$ and sodium phosphate dibasic $\left(\mathrm{Na}_{2} \mathrm{HPO}_{4}\right)$ were used of analytical grade. All aqueous solutions were prepared using deionized (DI) water. Phosphate buffer solution (PBS) (pH 7.8) (20mM) was used as the electrolyte solution, prepared using $\mathrm{NaH}_{2} \mathrm{PO}_{4}$ and $\mathrm{Na}_{2} \mathrm{HPO}_{4}$ salts. Colorimetric UA and Xanthine assay kits were purchased from ThermoFisher Sc. and Sigma Aldrich respectively and utilized to analyze clinical samples. PVA-SbQ purchased from Polysciences, Inc., was used to entrap UOx on SPCE purchased from $\mathrm{CH}$ Instruments, Inc., United States and the wound dressing. The flexible electrodes on wound dressing were screen printed using a 305-mesh screen using conductive graphite and $\mathrm{Ag} / \mathrm{AgCl}$ pastes (Gwent Group, UK) on adhesive vinyl (Silhouette America, USA).

The sensor was interfaced with LMP91000 miniaturized potentiostat having an analog front end (AFE), an ultra-low-power microcontroller (CC2650) integrated with a bluetooth module and LP2591 power management system from Texas Instruments (TI). A MCP72831charge controller, 12-bit digital-to-analog converter (DAC) with integrated electrically erasable programmable read-only memory (EEPROM) and a I2C compatible serial interface from Microchip formed part of the electronic circuit. 


\subsubsection{Apparatus and Methods}

A handheld BD-20AC Laboratory Corona Treater was utilized to treat the sensor surface for improved substrate functionalization. A schematic of the experimental setup is shown in Fig 5.1b. A goniometer was utilized to record contact angle measurements from the plasma treated sensor surface using sessile drop technique. This offered an improved understanding of sensor surface hydrophilicity. Standard electrochemical characterizations were carried out in a classical three-electrode system consisting of SPCE, external $\mathrm{Ag} / \mathrm{AgCl}$ as reference electrode and Pt counter electrode. Testing of the three electrode system was carried out in an electrochemical cell setup with $3 \mathrm{ml}$ electrolyte solution for both classical and flexible electrodes. Cyclic voltammetry (CV) and differential pulse voltammetry (DPV) using the analytical system model $\mathrm{CHI}-230 \mathrm{~B}$ potentiostat from $\mathrm{CH}$ Instruments, Inc. were conducted to evaluate the sensor performance. The ThermoFisher Sc. microplate absorbance reader was used to conduct assay experiments.

\subsubsection{Sweat sample collection protocol}

Before sample collection, a questionnaire was given to the subjects (Figure 5.3) to identify any health concern. 


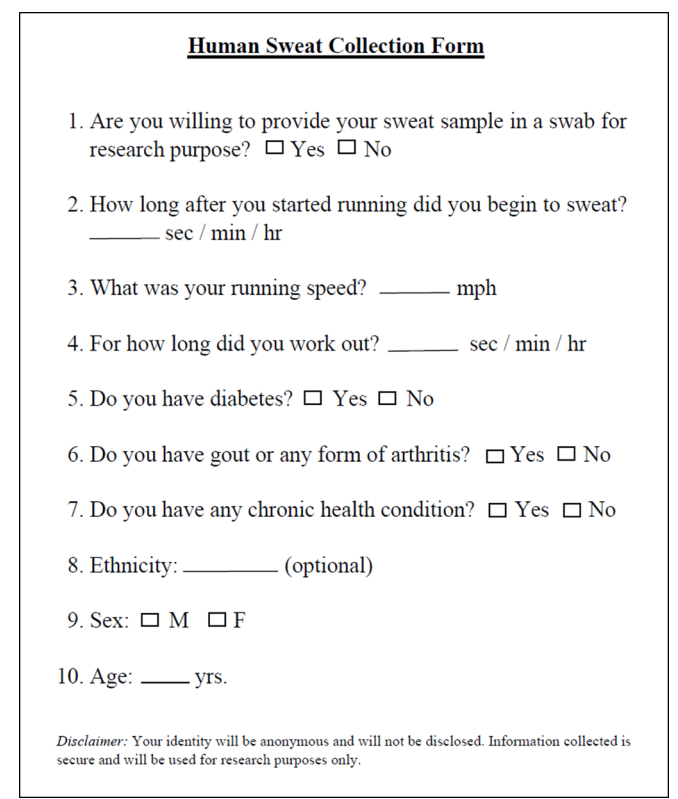

Figure 5.3 Survey form handed out to the volunteers from whom human sweat samples were collected [236]

Four samples were collected from subject volunteers between the age group of 25 to 35 . The subjects identified themselves as African American, Hispanic or Asian. These samples were collected as shown in Figure 5.4 under institutional review board (IRB) regulations.

Human sweat samples were collected from different healthy deidentified subjects after a run on a treadmill at a gym for 15 minutes. The 15 min duration was chosen because most of the subjects started sweating after $10 \mathrm{~min}$.

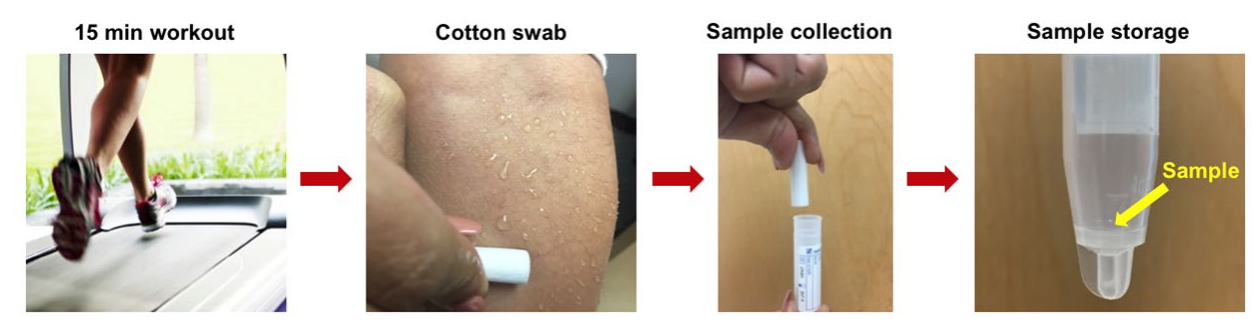

Figure 5.4 Steps involved in sweat sample collection [236] 


\subsubsection{Analyte extraction from wound dressing}

Multiple de-identified and discarded wound dressing samples were collected for UA and Xanthine extraction from patients inflicted with venous leg ulcers at the wound clinic in University of Miami. Two dressings, each about $10 \mathrm{~cm}$ x $10 \mathrm{~cm}$ in dimension, were collected from the subject's injured leg, placed at two different areas of his skin, at the time of dressing change. The first dressing was collected from one placed over the injured gash, while the second from one placed on a healthy region of skin, across from the one over the wound. Each of these wound dressings were carefully cut to $5 \mathrm{~cm} \times 5 \mathrm{~cm}$, as shown in Figure 5.5, to extract the biofluid that diffused through the dressings. Different

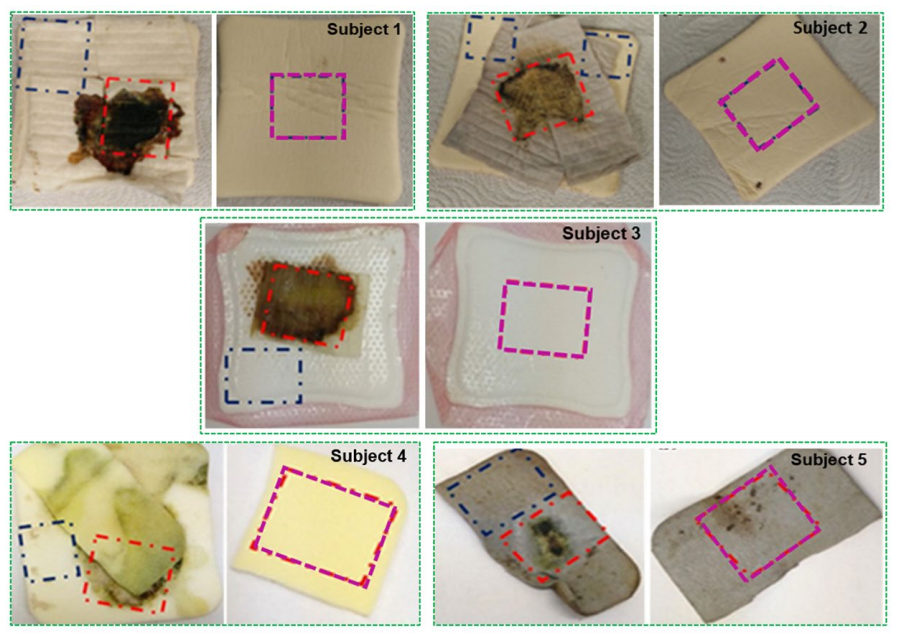

Figure 5.5 De-identified wound dressings from 5 patients, subjects 1-5 respectively are shown by green dashed lines (---); red dashed lines (---) represent the extraction area of wound exudate; blue dashed lines (---) represent the extraction area of biofluid from perilesional skin and pink dashed lines (---) represent healthy skin extracted area [244]

sections of each dressing as denoted in Figure 5.5, represent the extraction regions of biofluid from around wound proximity versus further away from it. Each of these samples were immersed in $5 \mathrm{ml} \mathrm{NaOH}$ solution prepared in DI water $(0.1 \mathrm{M}, \mathrm{pH} 12)$ and incubated at $37^{\circ} \mathrm{C}$ for forty-five minutes. They were then ultra-sonicated in a homogenizer for $60 \mathrm{~s}$ at $20 \mathrm{~Hz}$ and centrifuged for 5 minutes to separate the debris and extract the samples from the 
dressings. The extracted solutions of wound exudate and those from perilesional and healthy skin were tested on the biosensor. Assays for correlating concentrations of UA and Xanthine in different samples were conducted using standard colorimetric assay protocol at $570 \mathrm{~nm}$.

\subsubsection{Electrode fabrication on wearable construct}

Fabrication of electrodes on wound dressing was achieved through screen printing using conductive graphite paste on adhesive vinyl sheet with a $9 \times 14$ mesh screen. This graphite coated adhesive substrate was dried in nitrogen for 4 hours. Electrode design was

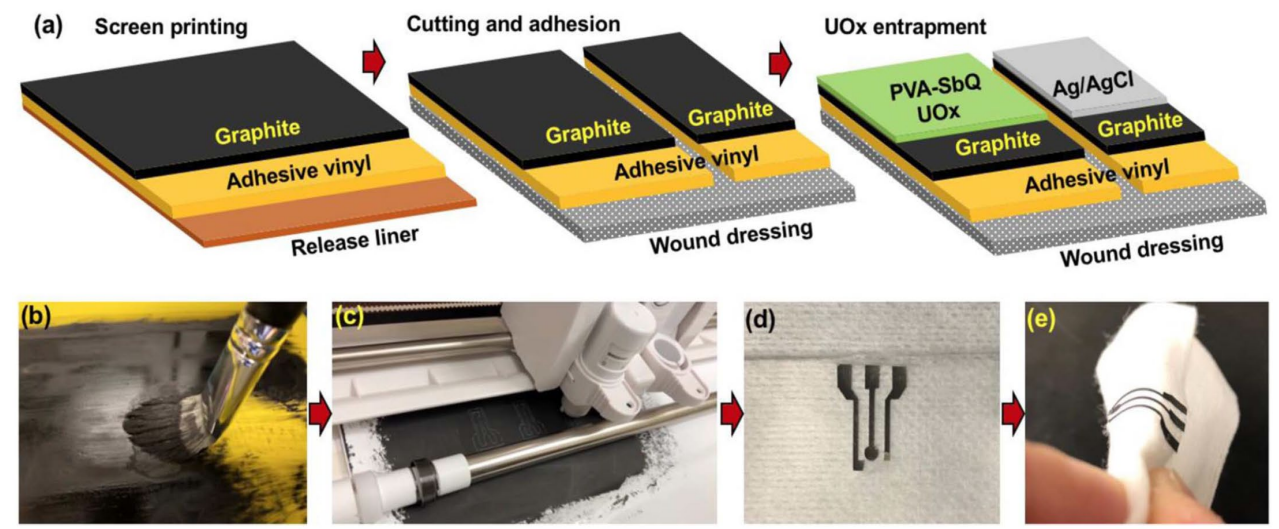

Figure 5.6 (a) Steps involved in IOT electrode preparation, (b) Screen printing using conductive graphite paste, (c) Precise cutting of electrode design, (d) Integration of flexible electrode on dressing, (e) Flexible electrode on wound dressing [211]

created on Silhouette Studio for precise cutting of the electrodes. This was then transferred onto flexible wound dressing. Conductive $\mathrm{Ag} / \mathrm{AgCl}$ paste was coated to create the reference electrode. The fabricated flexible electrode system on the wound dressing comprised of carbon working and counter electrodes and an $\mathrm{Ag} / \mathrm{AgCl}$ reference electrode. On the working electrode, the enzyme was entrapped using PVA-SbQ as mentioned previously in classical SPCE. These fabrication steps are schematically represented in Figure 5.6. 


\subsection{Effect of Corona Plasma on sensing performance}

The effect of Corona Plasma was investigated on the hydrophilicity of the sensor surface. As portrayed in Figure 5.7, a non-plasma treated electrode showed higher hydrophobicity with a contact angle of $118.3^{\circ}$. The presence of MWCNT/AuNP nanocomposite on the electrode reduced the contact angle to $115.1^{0}$. This was further seen to lower on plasma treatment to $64.8^{0}$. Incorporation of enzyme to the nanocomposite

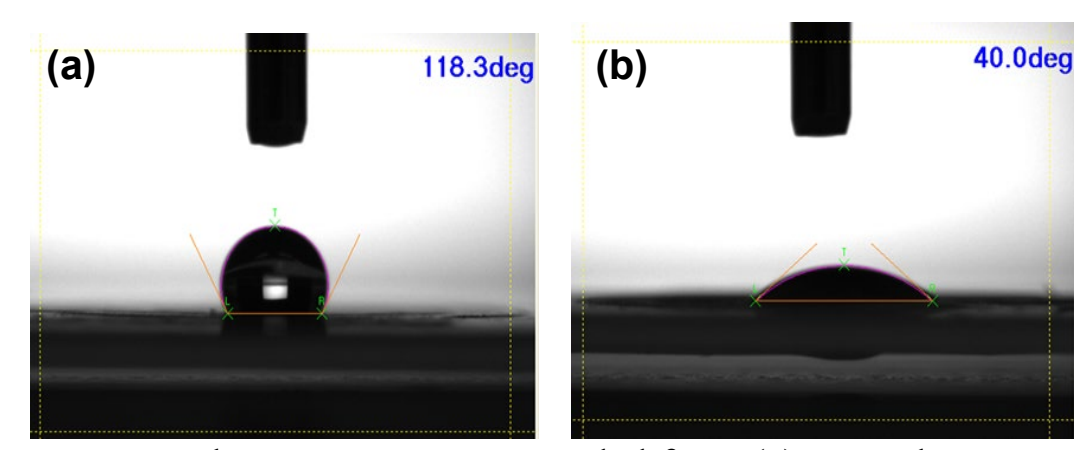

Figure 5.7 Contact angle measurements recorded from (a) Non-plasma treated and (b) Plasma treated electrodes

sensor surface showed a further decrease in contact angle to $40^{\circ}$. Hydrophilicity of the surface was thus seen to gradually increase with plasma treatment to the nanocomposite functionalized enzymatic sensor. This was determined from notable reduction in contact angle by $78.3^{0}$ as observed from Figure 5.7.

Effect of corona plasma was thereafter assessed on sensor performance using electrochemical measurements. Increased hydrophilicity of the sensor surface contributed to notably higher signal as illustrated from the CV plot depicted in Figure 5.8a. While a non-plasma treated electrode provided a current of about $0.2 \mu \mathrm{A}$, gradual increase in current was recorded from the plasma treated sensor surfaces. With a non-enzymatic sensor also providing higher signal on plasma treatment, further higher signal was recorded from the nanocomposite functionalized enzymatic sensor after being treated. An increase in 
current by about 5 -fold was recorded from the nanomaterial based enzymatic sensor surface as observed from Figure 5.8a.

This sensor was evaluated with different increasing concentrations of UA within a broad physiological range between $6 \mathrm{nM}$ and $0.4 \mathrm{mM}$. Linear increase in response was obtained at an $E \mathrm{p}_{\mathrm{a}}$ of about $0.3 \mathrm{~V}$ as seen from Figure 5.8b. With a low detection limit of

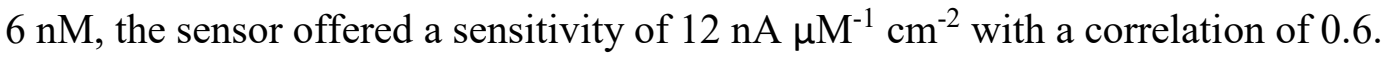
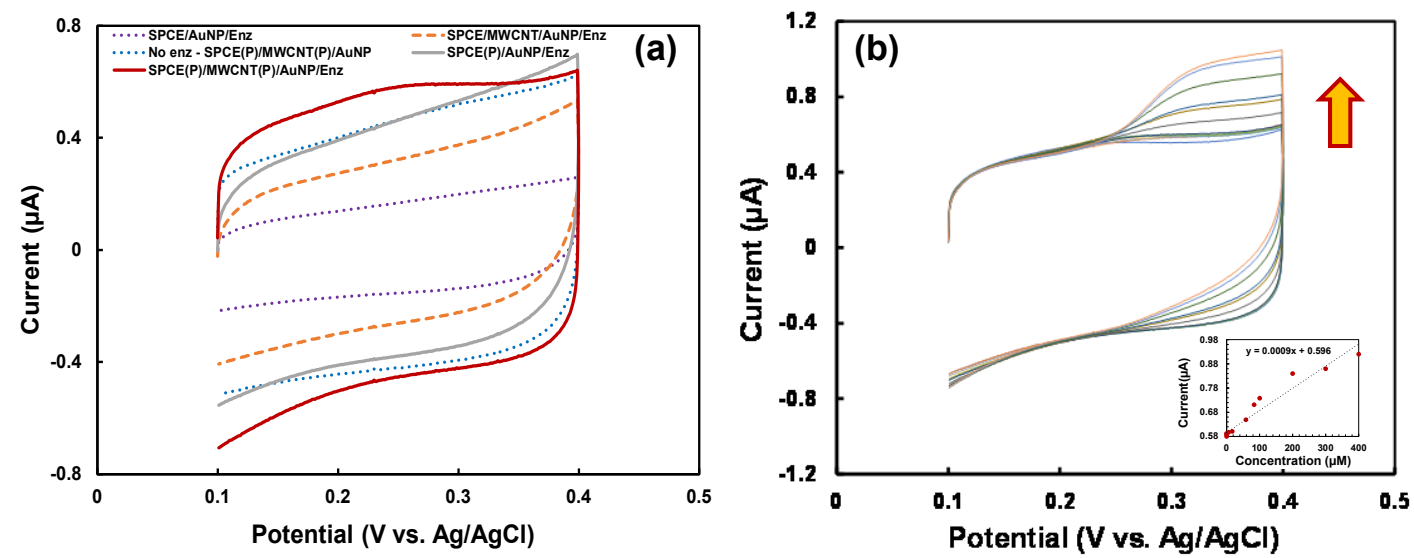

Figure 5.8 Plot depicting (a) CV response recorded from plasma treated and non-plasma treated electrodes in presence of UA, (b) Increase in response to different increasing concentrations of UA, inset: linear regression plot to increasing UA between $6 \mathrm{nM}$ and $0.4 \mathrm{mM}$

\subsection{Effect of sample volume on sensing performance}

Low sample production rates create insufficient liquid channels between the electrodes in a three-electrode system. Sufficient volume of analyte is thus necessary to facilitate charge and ion transport. The effects of different increasing sample volumes $(0.5$ to $50 \mu \mathrm{L})$ were tested using PDMS as a biocompatible substrate[227] for holding small volumes. (inset: Figure 5.9b). To enable uniform distribution of the sample on the electrode assembly, a sandwich setup was implemented. The setup includes a layer of PDMS containing the desired volume of sample, a wound dressing and then the transducer. For 

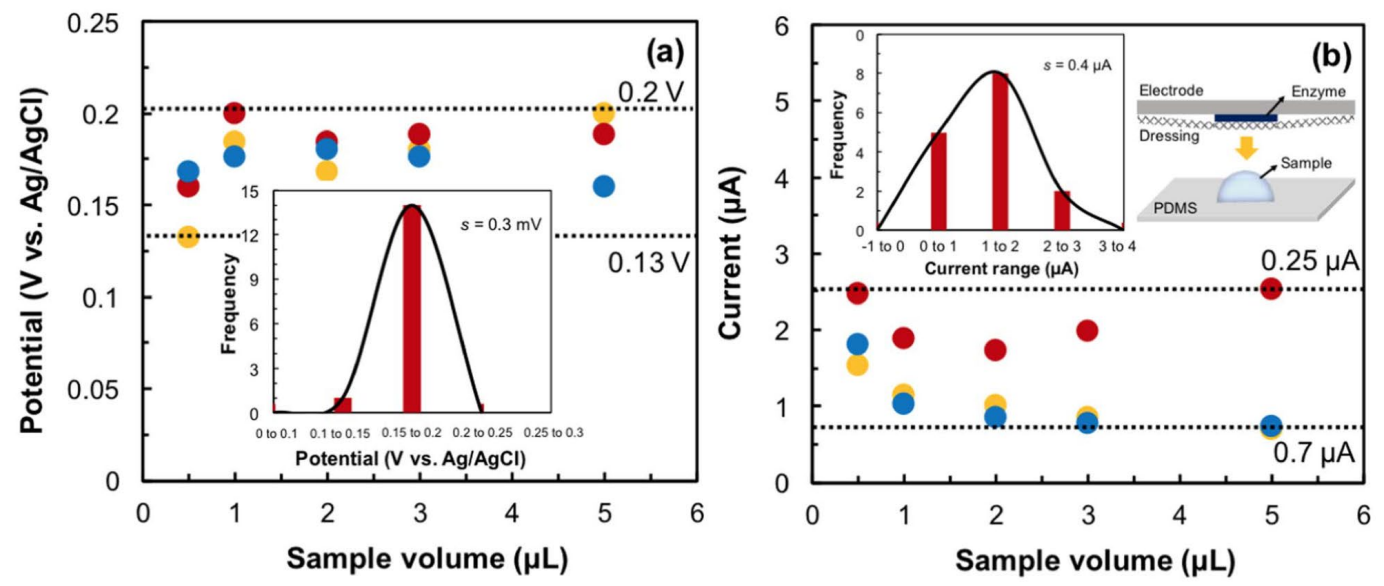

Figure 5.9 Effect of low sample volume on (a) $E \mathrm{p}_{\mathrm{a}}$ and (b) $I \mathrm{p}_{\mathrm{a}}$ of the biosensor, where $48 \mu \mathrm{M}$ uric acid was measured. The dotted lines in both graphs represent the upper and lower limits. The plots in the (a) and (b) insets are normal distribution curves representing potential and current, respectively. The inset schematic in (b) is the experimental setup [211]

small volume measures, the potential $\left(E \mathrm{p}_{\mathrm{a}}\right)$ falls in the range between $0.2 \mathrm{~V}$ and $0.13 \mathrm{~V}$ (Figure 5.9a). Normal distribution of this data provides $\sigma=0.3 \mathrm{mV}$, which demonstrates that low volumes do not affect the $E \mathrm{p}_{\mathrm{a}}$ of UA. Figure $5.9 \mathrm{~b}$ shows that $I \mathrm{p}_{\mathrm{a}}$ falls in the range between 0.25 to $0.7 \mu \mathrm{A}$ with a normal distribution, where $\sigma=0.4 \mu \mathrm{A}$. Measurements have indicated that this UA biosensor is sensitive enough to detect samples with volumes as low as $0.5 \mu \mathrm{L}$ (Figure 5.9). These studies accounted for low volumes present under the wound dressing in wound proximity of skin for real-time analysis. The measurements made have also examined for volumes that can spread evenly across the surface of the working electrode; which is enough to allow conduction of ions between the electrodes and to amplify signal effectively. 


\subsection{Clinical sample evaluation}

\subsubsection{Measurement of UA in sweat samples}

The biosensor was assessed by measuring UA levels present in human sweat obtained from de-identified samples collected for the study as discussed (Table 5.1- RS1 to RS4). These samples were measured by standard addition method in both UA assay and biosensor. A correlation between the assay and sensor measurements is drawn in Table 5.1. It can be seen from Table 5.1, that the UOx biosensor provided a varying recovery between $86.52 \%$ and $118.11 \%$ and an average RSD of $1.38 \%$. This shows the viability of noninvasive UA detection from human sweat samples.

\begin{tabular}{ccccc}
\hline Sample No. & Assay $(\boldsymbol{\mu M})$ & Biosensor $(\boldsymbol{\mu M})$ & Recovery $(\%)$ & RSD (\%) \\
\hline RS1 & 9.15 & 9.75 & 106.46 & 1.59 \\
RS2 & 29.46 & 34.80 & 118.11 & 2.19 \\
RS3 & 53.03 & 45.88 & 86.52 & 1.31 \\
RS4 & 64.30 & 63.95 & 99.46 & 0.44 \\
\hline
\end{tabular}

RS1, RS2, RS3 and RS4 represent the different sweat samples Table 5.1 Colorimetric UA assay vs. electrochemical detection of UA in human sweat samples using an enzymatic biosensor [236]

\subsubsection{Measurement of UA in extracted wound exudate}

As discussed in previous sections, healing progress can be monitored from correlations of UA concentrations in wound exudate. Extracted wound fluid from four different samples were analyzed using standard UA assay protocols to determine UA concentrations. The concentration values of the samples are given in Table 5.2, in the added column. To determine the performance of UOx biosensor in detection, electrochemical studies were performed to correlate these results with those obtained against standard colorimetric assay protocol. Different extracted wound fluid samples were tested against a 
linear calibration curve obtained from the standard assay concentrations. The concentrations values of UA obtained from the extracted samples of wound exudate are given in Table 5.2 - WF1 to WF4. Analysis from four de-identified patients with wounds of varying severity, provided us varying recovery of $85-150 \%$ and RSD of about (+/-) $5 \%$.

Sample No. Assay $(\mu M) \quad$ Biosensor $(\mu M) \quad$ Recovery (\%) $\quad$ RSD (\%)

\begin{tabular}{ccccc}
\hline WF1 & 76.64 & 65.21 & 85.09 & 4.64 \\
WF2 & 96.40 & 85.12 & 88.29 & 0.46 \\
WF3 & 106.40 & 115.83 & 108.86 & 3.91 \\
WF4 & 34.02 & 50.61 & 148.75 & 10.37 \\
\hline
\end{tabular}

WF1, WF2, WF3 and WF4 represent wound sample extracts from discarded dressings Table 5.2 Colorimetric UA assay vs. electrochemical detection of UA in extracted wound fluid of de-identified patients using an enzymatic biosensor [236]

Wound and treatment variations for each patient may vary owing to different levels of electrolytes and proteins. At any given time, debridement oozing through dressings in multiple patients may have varying levels of components (electrolytes, lactate, glucose, and proteins).[159, 199-202, 223, 227-228] Some deviation in response can thus be ascribed to these differences in wound fluid or sweat composition. For both sweat and wound extracts, each sample was assessed thrice. The mean of these values have been provided in Table 5.1 and Table 5.2. The intrinsic characteristics of the CV response obtained from a sweat and a wound sample extract have been shown in comparison with buffer (absence of sample) in Figure 5.10. This portrays that higher current is obtained from a sample extract of wound exudate, therefore suggesting the presence of elevated UA 
concentration in purulent wound fluid as compared to sweat, which contains relatively lower concentrations of the analyte.

This study establishes the detection of UA from human wound exudate and sweat, which have not been achieved before. Measurable results obtained from bio-fluids of sweat and wound pave the potential for electrochemical detection of UA in wound theranostics.

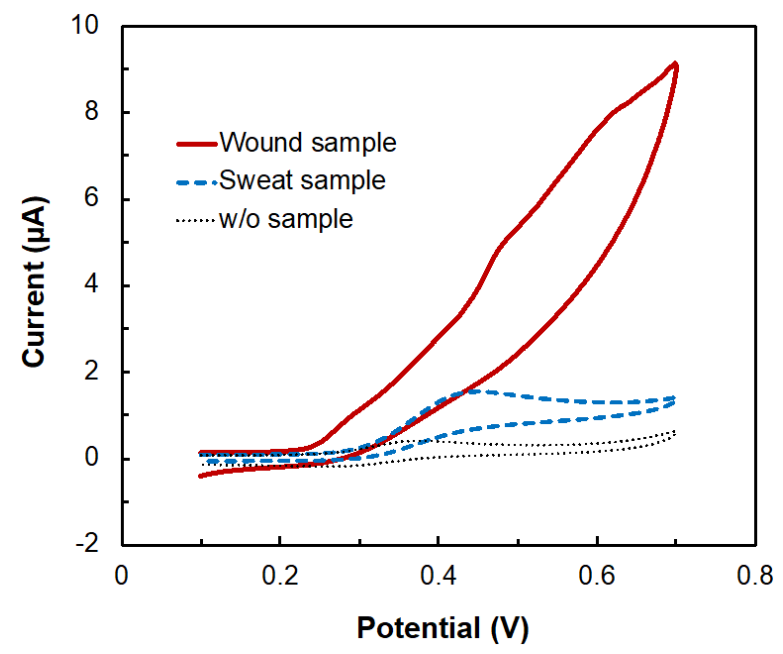

Figure 5.10 Sample plot depicting CV response of the UOx electrode to 1 of the sweat and wound samples, measured at $20 \mathrm{mV} \mathrm{s}^{-1}$. This data shows wound has higher concentration of uric acid than sweat as reported [236]

Such a method may potentially facilitate non-invasive biomarker detection from sweat around a wound, thus preventing occlusion effects from other contaminants in concentrated wound fluid when embedding the sensor directly on the wound.

\subsubsection{Effect of sweat lipid emulsification on sensor signal}

Measuring UA form sweat will be influenced by the composition of sweat metabolites. Secreted from eccrine glands of the skin, sweat helps regulate body temperature. It is mainly composed of water with dissolved minerals, proteins, and ions.[229] At any given time, sweat secreted by different people may have varying levels 
of these components. This kind of difference in content could trigger changes in biosensor measurements.

The biosensor was tested to detect UA in sweat using standard addition method with samples prepared by spiking known concentrations of UA and testing them against its linear calibration curve. Two different sources were selected for this study, one clearer than the other. Increased turbidity in sweat could result from emulsification of lipid/fat molecules in certain cases.[230] Such variations affect the electrochemical signal. Results showed that the clearer sample provided a positive recovery while the turbid sample provided a negative one. This negative value can be attributed to fat molecules blocking

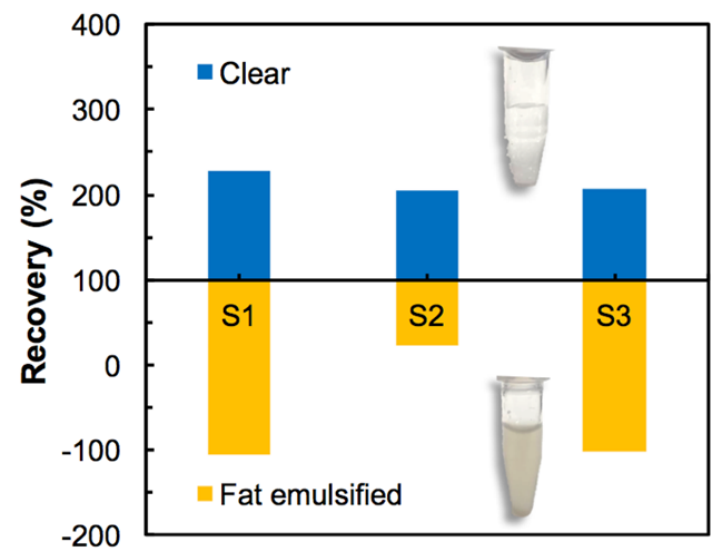

Figure 5.11 Plot representing the effect of fat emulsification in sweat on the biosensor recovery data obtained through DPV technique. S1, S2 and S3 represent 30.3, 33.9 and $37.5 \mu \mathrm{M}$ uric acid respectively. The top and bottom vial images are the clear and fat emulsified sweat samples

the electrode surface. Emulsification of lipid molecules could thus be considered as a cause of change in recovery. These results denoting recovery of UA from the sweat samples are plotted as seen in Figure 5.11. 


\subsubsection{Clinical sample evaluation for Xanthine}

Venous leg ulcers and sickle cell disease leg ulcers of de-identified patients within a week of therapy were analyzed (Figure 5.12). Therapy for such wounds is primarily compression and pressure oriented, to increase the venous return and reduce venous hypertension to allay the pressure damage caused in valves of leg veins.[231-232] Analysis from these dressings showed, that the wound exudate from larger wounds $\left(14 \mathrm{~cm}^{2}\right)$ had 3.25 $\mu \mathrm{M}$ Xanthine compared to 2.77 and $1.85 \mu \mathrm{M}$ for wounds with an area of $5 \mathrm{~cm}^{2}$ and 1 $\mathrm{cm}^{2}$, respectively. Such a relationship suggests that a correlation may be drawn between wound severity and Xanthine concentrations;[233] where wounds with a larger area contain higher concentrations of Xanthine than smaller wounds. A correlation can be drawn between severe wounds depicting higher analyte concentrations than healing wounds, with large wounds typically taking longer to heal.[234-235] However, larger number of sample studies are necessary to validate these correlations.
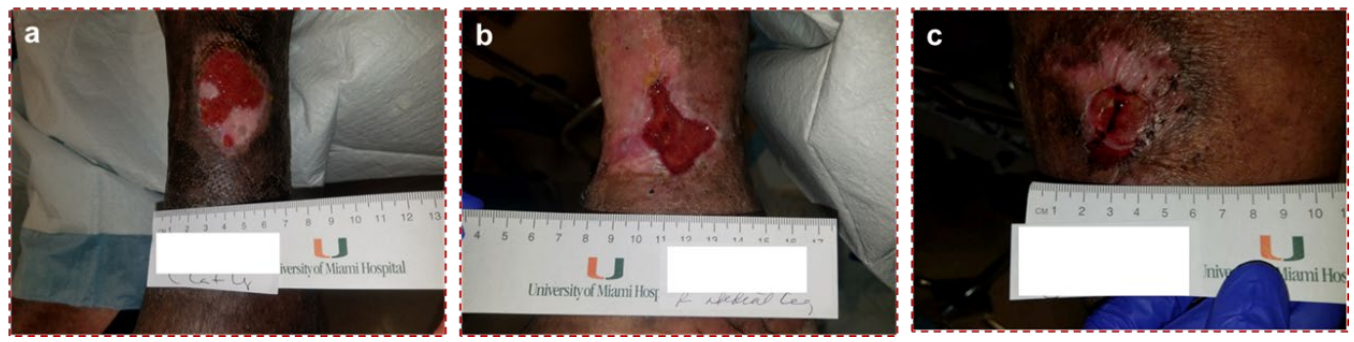

Figure 5.12 De-identified wound images of patients diagnosed with venous leg ulcers $(a, b)$ and sickle cell disease leg ulcer (c) [244] 
To assess the ability of Xanthine detection from wound vicinity non-invasively, extracted biofluids from perilesional and healthy skin were measured for the presence of Xanthine, along with exudate extracted from wound dressings of five different patients. The biosensor was evaluated against standard colorimetric assay protocols for Xanthine to analyze the extracted bio-fluids and determine the concentrations of Xanthine. Results obtained from this study have been depicted in Table 5.3 and Figure 5.13.

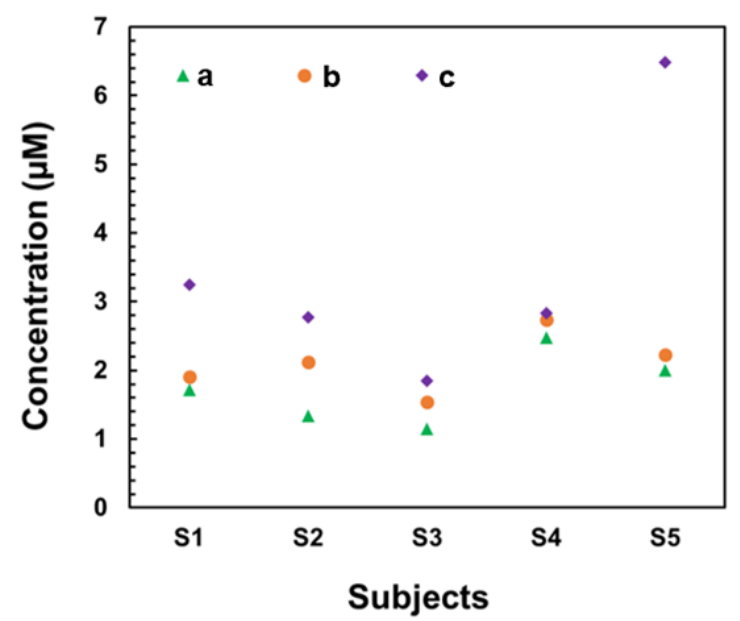

Figure 5.13 Concentrations of xanthine concentrations from extracts of biofluids sampled from (a) healthy skin, (b) perilesional skin and (c) wound exudate of five deidentified patients using the biosensor [244]

Analyte concentrations were observed to be higher from extracted wound exudate than from further away from the injury site. Biofluids from healthy skin showed comparatively lower concentrations. This indicates that concentrations of xanthine are higher in wound proximity and are reduced when further away from the lesion site. A correlation between the assay and sensor measurements as portrayed in Table 5.3, shows that the biosensor readings have high deviations for wound exudate measurements as compared to measurements from perilesional and healthy skin. These deviations are reflected in their recovery values as seen in the same table, where wound exudate has about $122 \%$ deviation, while extracts from perilesional and healthy skin have lower deviations 
of approximately $40.48 \%$ and $86.41 \%$, respectively. This deviation in response can be accredited to differences from the presence of various electrolytes and proteins in the biofluid matrices.

This study provided an understanding of the concentration gradient of the bioanalyte in wound proximity. On the occurrence of a wound, fluid oozes in and around the punctured tissue by diffusing through glands and surrounding tissue through the internal, lower dermal layers of skin due to changes in osmotic gradients of the metabolites.[236] Therefore, the area around the wound also has a rise in analyte concentrations. It can be seen from this study that measuring Xanthine from biofluids in and around the injury of a person is possible and not at all cumbersome. This shall also allow reduction of occlusion from embedding the sensor directly on the wound.

\subsubsection{Effect of biofluid occlusion on sensor surface}

The effect of biofluid occlusion on the active surface of the biosensor was studied through electrochemical analysis. It showed the rate of degrade in response to sequential deposition of extracts of wound exudate and biofluids from around the wound. The sensor showed a gradual decrease in response to extracts from perilesional and healthy skin with the same volume of analyte. As illustrated in Figure 5.14, the rate of decrease in response to wound exudate was $20 \%$ more than when subjected to extracts from around and away from the wound. This higher rate of decrease can be attributed to the occlusion of solid proteins, proteases, cytokines and neutrophils present in concentrated wound exudate, on the active area of the electrode. Such accumulation tends to block the electrode surface, limiting the diffusion of analyte to the transducer and promoting electrode fouling. Measurements recorded over different sequential deposition of analyte have shown that the 
response of the enzymatic electrode was reduced by only $10 \%$ in the presence of biofluid from healthy skin before attaining stability. It can thus be said that the enzymatic biosensor could potentially be utilized for wound healing therapy through assessment of Xanthine levels from biofluids in injured vicinity.

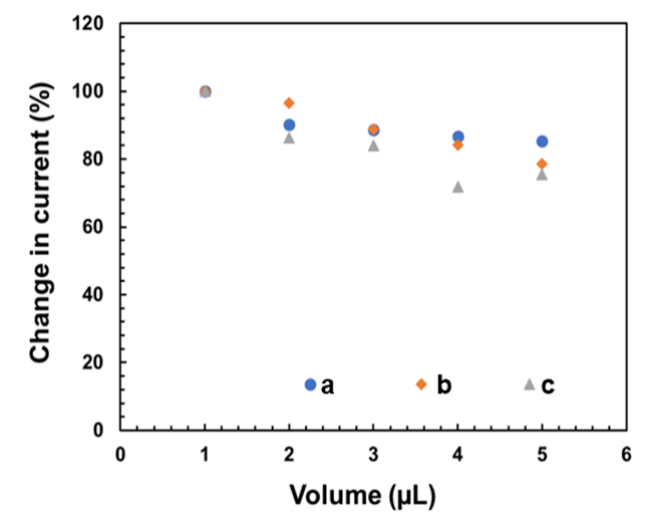

Figure 5.14 Plot depicting percentage change in current response of the biosensor as a function of volume of extracted biofluids from (a) healthy skin, (b) perilesional skin and (c) wound exudate [244]

\subsection{Electrochemical sensing performance on wearable platform}

Sensing operation from wearable platform - The enzymatic sensor designed on cotton gauze was interfaced with an analog front end (AFE), LMP91000 on a flexible platform. The customized printed circuit board (PCB) was manufactured on flexible Kapton tape with a two-layer board $\sim 800$ microns thick (Figure 5.15a). It was designed to accommodate the low-cost potentiostat (LMP91000) with a low power data processing microcontroller, CC2650 integrated with a bluetooth module for wireless data transmission. 
A schematic of the potentiostat is given in Figure 5.15b. The arrangement of the circuit is that of a non-inverting operational amplifier. LMP91000 was configured via the microcontroller, CC2650 to perform three electrode amperometry according to the schematic in Figure 5.15b. Microcontroller, CC2650 was used as an integrated wireless microcontroller with bluetooth low energy (BLE) capabilities to provide wireless communication and peripheral controls remotely at ultra-low power cost-effectively with its $2.4 \mathrm{GHz}$ transceiver. It was connected to the LMP via the $\mathrm{I} 2 \mathrm{C}$ interface, as in Figure $5.15 \mathrm{c}$.

(a)

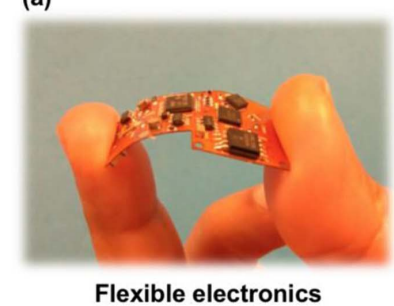

Flexible electronics (b)

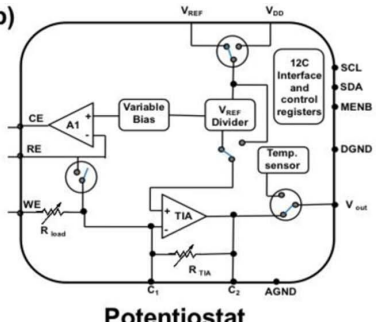

(c)

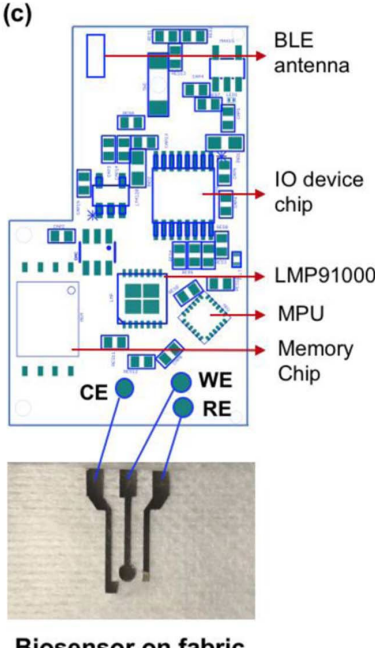

Biosensor on fabric

Figure 5.15 (a) Flexible electronics; (b) Schematic of the potentiostat and connections; (c) Circuit design of components embedded on the PCB interfaced with the flexible wound sensor [211]

As depicted in Figure 5.15b, amplifier A1 is the control amplifier that implements the potentiostat circuit. The variable bias block provides a constant potential across the working and reference terminals of A1. The voltage supplied by the source was closely followed by the voltage between the reference and working electrodes. All three electrodes on the dressing were connected to corresponding pins of the AFE, linked to the microcontroller, CC2650 as shown in Figure 5.15c. Electronic components including the microcontroller, AFE, power management systems with BLE Antenna, I/O device chip, 
MPU, memory chip and associated circuitry were mounted on the top layer as shown in Figure 5.15c.

The transition from minimal current flow to voltage was made available by the trans-impedance amplifier (TIA) whose forward voltage gain depends on feedback resistor $R_{\mathrm{tia}}$, connected to the feedback path of the TIA. This converts the current flowing between the counter and working electrodes to a proportional voltage, connected to $V_{\text {out }} V_{\text {out }}$ from the LMP91000 was routed to the microcontroller general purpose input/output, where it was conditioned by an internal analog-to-digital converter for interpretation. The internal feedback resistor was optimized for optimal amplifier gain through $R_{\text {tia }}=120 \mathrm{k} \Omega$, which offered large enough gain to allow for voltage fluctuations owing to changes in analyte concentrations. The reference voltage $\left(V_{R E F}\right)$ to the AFE sensing device was externally provided by the digital to analog converter. The developed wearable platform operated on a 3.7 $V_{\mathrm{DC}}$ lithium-ion (Li-ion) battery capable of providing up to $350 \mathrm{mAh}$. The Li-ion battery used can be recharged on the wearable platform through a micro-USB device connected directly onto a charging circuit allowing simultaneous system operation and charging. Battery voltage was regulated to provide a constant $0.3 V_{\mathrm{DC}}$ source to the system in through a battery voltage range of $3 V_{\mathrm{DC}} \leq V_{\mathrm{DC}} \leq 3.7 V_{\mathrm{DC}}$. When bluetooth capabilities were enabled by the user, the current consumption of the system increased from 2 to $\sim 40$ $\mathrm{mA}$. This current generated by the potentiostat from the working electrode, corresponds to the concentration of the analyte. [211]

Power consumption of the device depends not only on the amperometric operation of the potentiostat, but also on the BLE transmission and communication; along with the run time current drawn from the central processing unit (CPU). Since most of these 
operations do not simultaneously run except in emergency mode, the modules running them could be pushed to a low power state, reducing consumption. For precise calibration, the computational power being demanding on all devices, processing of information can be performed on the cloud on data upload. This saves battery power and comparatively limits computational power over the device and low energy Bluetooth. While the CPU runs for a shorter duration during BLE transmission and conversion of the analog output from the potentiostat, the remaining time can be used for other peripheral operations consuming an average power of $\sim 56 \mu \mathrm{W}$ over a $3.7 \mathrm{~V}$ and $350 \mathrm{mAh}$ battery, providing a system operational lifetime of multiple days. [211]

Response from wearable construct - Response from a classical electrode setup was compared to that obtained from the sensor designed on gauze. With a mean response of about $4.27 \mu \mathrm{A}$, a standard deviation of $0.6 \mu \mathrm{A}$ was observed as depicted in Figure. 5.16a. A slight shift in potential was also noticed with a standard deviation of $0.01 \mathrm{~V}$ as seen in Figure. 5.16b. This information was then sent wirelessly via bluetooth to the receiver which possesses abilities to send the data to the cloud. Information processed and sent from the
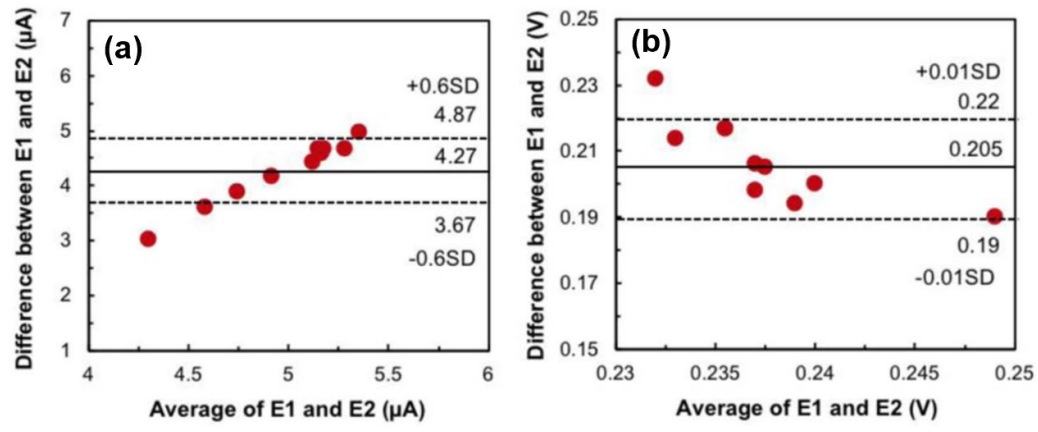

Figure 5.16 Bland-Altman plots representing variation in (a) current and (b) potential from studies using classical setup and IoT platform in presence of $100 \mu \mathrm{M}$ UA, measured at $20 \mathrm{mV} \mathrm{s}^{-1}$. E1 and E2 represent the classical SPCE and biosensor on fabric respectively [211] 
bluetooth module to the receiving device was encrypted to avoid data compromise and enable secure transmission.[237]

Such a system could therefore enable patient-physician remote monitoring.[238] Analytical tools could be built on the cloud for data analysis of corresponding wound biomarker concentrations to monitor wound severity and healing progress.

\subsection{Conclusion}

Enzymatic potentiometric biosensors were designed on cotton gauze for wearable monitoring of wound biomarkers, UA and Xanthine. The detection of UA and Xanthine from extracted biofluids of wound exudate and sweat was established for the first time through this study. Detection from bio-fluid matrices around an injured site was evaluated, with portrayed higher concentrations in wound proximity than further away. Possibility of non-invasive detection from wound milieu was explored to show the feasibility of biomarker detection from wound proximity. Results have shown a correlation between wound dimensions and analyte concentrations. This study paves the potential for detection of purine metabolites from biofluids in and around the wound, on a wearable, healing platform. This shall enable non-invasive assessment of lesion severity, providing quantitative insights on healing progress. 


\section{CHAPTER 6}

\section{SUMMARY AND FUTURE DIRECTION}

\subsection{Summary}

Point-of-care techniques, entailing the detection of specific biomolecules can enable quantitative monitoring of a wound's healing progress. As an attempt to transform woundcare with continuous monitoring of healing and effective wound management, this dissertation discusses the feasibility of a biosensing system on a wearable, healing platform. A bio-sensing system that can electrochemically detect analytes like UA and Xanthine can potentially evaluate the healing progress of a wound, bearing correlations with wound severity.

Methods to stabilize the sensing performance have been implemented through specific sensor surface modifications and robust enzyme immobilization protocols in sensor design. The use of nanomaterial in sensor design was assessed using a nanocomposite of MWCNT/AuNP. Enhanced sensing performance was noted from increased active surface area and binding sites offered by the conductive nano-composite, which enabled higher loading of enzyme and firm immobilization. The use of Corona Plasma was noted to improve sensing response with increased hydrophilicity, enabling greater surface functionalization. Stability and shelf life of the biosensor over multiple days was assessed under relevant physiological variations of temperature and $\mathrm{pH}$ using electrochemical techniques. The validation of sensor performance in extracts from bio-fluid matrices associated with an injury site was performed using electrochemical and colorimetric approaches. Viability of detection of UA and Xanthine from these extracts of relevant 
biofluids was conducted to observe correlations between wound severity and the concentrations of UA and Xanthine.

\subsection{Prospects}

Existing wound care being expensive can cause stress and inconvenience to patients. The importance of patient compliance by health professionals is crucial and can make a huge difference between optimal wound healing and unwanted health issues. Wearable biosensing for wound management offers promise in cost reduction and convenience for the patient through smart technology, minimizing exacerbation. [211]

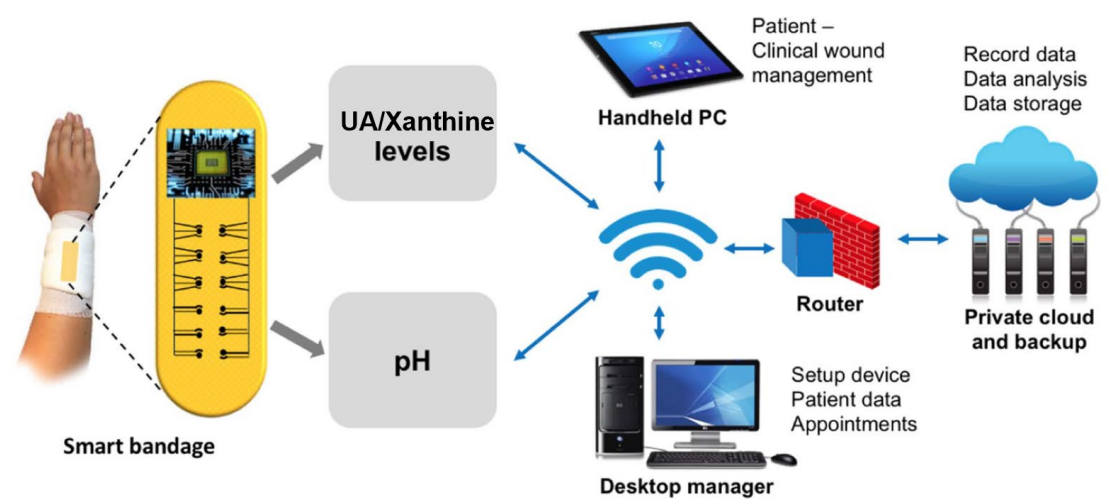

Figure 6.1 Schematic representing the convergence of smart wound care management and IOT [211]

Of late, the rise of digital medicine is revolutionizing traditional laboratory-based treatment protocols with access to real-time patient data and remote patient monitoring in personal health management.[239] The advent of the medical IoT, will enable real-time continuous tracking of healing through miniaturized, remote sensing. This evolution presents enormous potential for convenient, personalized wound care, which can assist in taking medical decisions through the healing stages as and when necessary. In this age of 
healthcare, a network of devices and appliances embedded with electronics, software and sensors, connected to exchange data, can create breakthroughs in personalized medicine.

A biosensor to monitor wound biomarkers shows the possibility of continuous monitoring of lesion healing in physiologically relevant conditions. Correlation of analyte levels with $\mathrm{pH}$ and temperature in an injured environment, can enable calibration of response through sensor fusion. Figure 6.1 depicts the convergence of wound management with medical IoT. Advancing toward personalized wellness evaluation, the sensor complements comprehensive wound management on a flexible, wound-care platform. This sensing system as shown in Figure 6.1, will allow remote patient-physician engagements[240], enable setting up of appointments and validation of treatment based on data acquired at a personal scale. Such tools will provide powerful wound care solutions for effective therapy to continuously monitor recovery progress and detect chronicity through rapid analyses from wound milieu. 


\section{REFERENCES}

1. Shafiee, H. et al. Paper and flexible substrates as materials for biosensing platforms to detect multiple biotargets. Sci. Rep. 5, 8719 (2015).

2. Bao, Z. \& Chen, X. Flexible and Stretchable Devices. Adv. Mater. 28, 4177-4179 (2016).

3. Joo Chuan Yeo, K. \& Teck Lim, C. Emerging flexible and wearable physical sensing platforms for healthcare and biomedical applications. Microsystems Nanoeng. 2, $1-$ 19 (2016).

4. Zhao, Y. \& Huang, X. Mechanisms and Materials of Flexible and Stretchable Skin Sensors. Micromachines 8, 69 (2017).

5. Rocchitta, G. et al. Enzyme Biosensors for Biomedical Applications: Strategies for Safeguarding Analytical Performances in Biological Fluids. Sensors (Basel). 16, 780 (2016).

6. Grieshaber, D., MacKenzie, R., Vörös, J. \& Reimhult, E. Electrochemical Biosensors - Sensor Principles and Architectures. Sensors (Basel). 8, 1400-1458 (2008).

7. Islam, T. \& Mukhopadhayay, S. C. Wearable sensors for physiological parameters measurement: physics, characteristics, design and applications. (IOP Publishing Ltd., 2017). doi:10.1088/978-0-7503-1505-0ch1

8. Sheth, A., Jaimini, U. \& Yip, H. Y. How Will the Internet of Things Enable Augmented Personalized Health? IEEE Intell. Syst. 33, 89-97 (2018).

9. Appelboom, G. et al. Smart wearable body sensors for patient self-assessment and monitoring. Arch. Public Health 72, 28 (2014).

10. Dias, D. \& Paulo Silva Cunha, J. Wearable Health Devices-Vital Sign Monitoring, Systems and Technologies. Sensors (Basel). 18, (2018).

11. Peake, J. M., Kerr, G. \& Sullivan, J. P. A Critical Review of Consumer Wearables, Mobile Applications, and Equipment for Providing Biofeedback, Monitoring Stress, and Sleep in Physically Active Populations. Front. Physiol. 9, 743 (2018).

12. Heikenfeld, J. et al. Wearable sensors: modalities, challenges, and prospects. Lab Chip 18, 217-248 (2018).

13. Lu, F. et al. Review of Stratum Corneum Impedance Measurement in Non-Invasive Penetration Application. Biosensors 8, 1-20 (2018). 
14. Luis, J., Luna, V., Krenn, M., Cortés Ramírez, J. A. \& Mayr, W. Dynamic Impedance Model of the Skin-Electrode Interface for Transcutaneous Electrical Stimulation. PLoS One 10, 1-15 (2015).

15. J. S. Son, US Pat., US20170010, 2013.

16. Liu, Y. et al. Flexible, Stretchable Sensors for Wearable Health Monitoring: Sensing Mechanisms, Materials, Fabrication Strategies and Features. Sensors 18, 645 (2018).

17. Farooq, M. \& Sazonov, E. Strain Sensors in Wearable Devices. in Wearable Electronics Sensors 221-239 (Springer, Cham, 2015). doi:10.1007/978-3-31918191-2_9.

18. Zhang, S.-H. et al. Wearable Wide-Range Strain Sensors Based on Ionic Liquids and Monitoring of Human Activities. Sensors (Basel). 17, (2017).

19. Lee, S. P. et al. Highly flexible, wearable, and disposable cardiac biosensors for remote and ambulatory monitoring. npj Digit. Med. 1, 1-8 (2018).

20. Wang, J. \& Rogers, K. Electrochemical Sensors For Environmental Monitoring: A Review Of Recent Technology. (1995).

21. Gao, W. et al. Fully integrated wearable sensor arrays for multiplexed in situ perspiration analysis. Nature 529, 509-514 (2016).

22. Lee, H., Hong, Y. J., Baik, S., Hyeon, T. \& Kim, D.-H. Enzyme-Based Glucose Sensor: From Invasive to Wearable Device. Adv. Healthc. Mater. 7, 1701150 (2018).

23. Karpova, E. V. et al. Noninvasive Diabetes Monitoring through Continuous Analysis of Sweat Using Flow-Through Glucose Biosensor. Anal. Chem. 91, 37783783 (2019).

24. Bhide, A., Muthukumar, S., Saini, A. \& Prasad, S. Simultaneous lancet-free monitoring of alcohol and glucose from low-volumes of perspired human sweat. Sci. Rep. 8, 6507 (2018).

25. Lee, H. et al. Wearable/disposable sweat-based glucose monitoring device with multistage transdermal drug delivery module. Sci. Adv. 3, e1601314 (2017).

26. Jia, W. et al. Electrochemical Tattoo Biosensors for Real-Time Noninvasive Lactate Monitoring in Human Perspiration. Anal. Chem. 85, 6553-6560 (2013).

27. Currano, L. J. et al. Wearable Sensor System for Detection of Lactate in Sweat. Sci. Rep. 8, 15890 (2018). 
28. Anastasova, S. et al. A wearable multisensing patch for continuous sweat monitoring. Biosens. Bioelectron. 93, 139-145 (2017).

29. Imani, S. et al. A wearable chemical-electrophysiological hybrid biosensing system for real-time health and fitness monitoring. Nat. Commun. 7, 11650 (2016).

30. Nyein, H. Y. Y. et al. A Wearable Electrochemical Platform for Noninvasive Simultaneous Monitoring of $\mathrm{Ca}^{2+}$ and pH. ACS Nano 10, 7216-7224 (2016).

31. Guinovart, T., Bandodkar, A. J., Windmiller, J. R., Andrade, F. J. \& Wang, J. A potentiometric tattoo sensor for monitoring ammonium in sweat. Analyst 138, 7031 (2013).

32. Bandodkar, A. J. et al. Epidermal tattoo potentiometric sodium sensors with wireless signal transduction for continuous non-invasive sweat monitoring. Biosens. Bioelectron. 54, 603-609 (2014).

33. Schazmann, B. et al. A wearable electrochemical sensor for the real-time measurement of sweat sodium concentration. Anal. Methods. 2, 342-348 (2010).

34. Parrilla, M., Ferré, J., Guinovart, T. \& Andrade, F. J. Wearable Potentiometric Sensors Based on Commercial Carbon Fibres for Monitoring Sodium in Sweat. Electroanalysis 28, 1267-1275 (2016).

35. Guo, S. \& Dipietro, L. A. Factors affecting wound healing. J. Dent. Res. 89, 219$29(2010)$.

36. Chiang, B. et al. Enhancing skin wound healing by direct delivery of intracellular adenosine triphosphate. Am. J. Surg. 193, 213-8 (2007).

37. Fernandez, M. L., Upton, Z. \& Shooter, G. K. Uric Acid and Xanthine Oxidoreductase in Wound Healing. Curr. Rheumatol. Rep. 16, 396 (2014).

38. Ridiandries, A., Tan, J. T. M. \& Bursill, C. A. The Role of Chemokines in Wound Healing. Int. J. Mol. Sci. 19, 1-20 (2018).

39. Martillo, M. A., Nazzal, L. \& Crittenden, D. B. The crystallization of monosodium urate. Curr. Rheumatol. Rep. 16, 400 (2014).

40. Zhou, Y., Zhao, M., Pu, Z., Xu, G. \& Li, X. Relationship between oxidative stress and inflammation in hyperuricemia. Medicine (Baltimore). 97, e13108 (2018).

41. National Institute of Diabetes and Digestive and Kidney Disease. Diabetic neuropathies: The nerve damage of diabetes. http://www.niddk.nih.gov/healthinformation/health-topics/Diabetes/diabetic-neuropathies-nerve-damagediabetes/Pages/diabetic-neuropathies-nerve-damage.aspx. 
42. Sen, C. K. et al. Human skin wounds: a major and snowballing threat to public health and the economy. Wound Repair Regen. 17, 763-71 (2009).

43. Mundaca-Uribe, R. et al. Development of a bienzymatic amperometric biosensor to determine uric acid in human serum, based on mesoporous silica (MCM-41) for enzyme immobilization. Sensors Actuators B Chem. 195, 58-62 (2014).

44. Dargaville, T. R. et al. Sensors and imaging for wound healing: A review. Biosens. Bioelectron. 41, 30-42 (2013).

45. Fernandez, M. L., Upton, Z., Edwards, H., Finlayson, K. \& Shooter, G. K. Elevated uric acid correlates with wound severity. Int. Wound J. 9, 139-149 (2012).

46. Dreifke, M. B., Jayasuriya, A. A. \& Jayasuriya, A. C. Current wound healing procedures and potential care. Mater. Sci. Eng. C. Mater. Biol. Appl. 48, 651-62 (2015).

47. Pasalic, D., Marinkovic, N. \& Feher-Turkovic, L. Uric acid as one of the important factors in multifactorial disorders--facts and controversies. Biochem. medica 22, 6375 (2012).

48. Honeychurch, K. The Determination of Uric Acid in Human Saliva by Liquid Chromatography with Electrochemical Detection. J. Anal. Bioanal. Sep. Tech. 2, 47-51 (2017).

49. Kim, J. et al. Wearable salivary uric acid mouthguard biosensor with integrated wireless electronics. Biosens. Bioelectron. 74, 1061-1068 (2015).

50. Desideri, G. et al. Is it time to revise the normal range of serum uric acid levels? Eur. Rev. Med. Pharmacol. Sci. 18, 1295-306 (2014).

51. de Oliveira, E. P. \& Burini, R. C. High plasma uric acid concentration: causes and consequences. Diabetol. Metab. Syndr. 4, 12 (2012).

52. Sila-On, A., Pavaro, U. \& Nuchpramool, W. Serum and urinary uric acid levels in healthy subjects and in patients with urolithiasis. J. Med. Assoc. Thai. 74, 352-7 (1991).

53. Horwath-Winter, J. et al. Determination of uric acid concentrations in human tear fluid, aqueous humour and serum. Acta Ophthalmol. 87, 188-192 (2009).

54. Wu, D. et al. Uricase-stimulated etching of silver nanoprisms for highly selective and sensitive colorimetric detection of uric acid in human serum. Sensors Actuators B Chem. 221, 1433-1440 (2015). 
55. Zhang, F. et al. Immobilization of uricase on $\mathrm{ZnO}$ nanorods for a reagentless uric acid biosensor. Anal. Chim. Acta 519, 155-160 (2004).

56. Yu, J., Wang, S., Ge, L. \& Ge, S. A novel chemiluminescence paper microfluidic biosensor based on enzymatic reaction for uric acid determination. Biosens. Bioelectron. 26, 3284-3289 (2011).

57. Perelló, J., Sanchis, P. \& Grases, F. Determination of uric acid in urine, saliva and calcium oxalate renal calculi by high-performance liquid chromatography/mass spectrometry. J. Chromatogr. B 824, 175-180 (2005).

58. Siekmann, L. Determination of uric acid in human serum by isotope dilution-mass spectrometry. Definitive methods in clinical chemistry, III. J. Clin. Chem. Clin. Biochem. 23, 129-35 (1985).

59. Cano Sanchez, M., Lancel, S., Boulanger, E. \& Neviere, R. Targeting Oxidative Stress and Mitochondrial Dysfunction in the Treatment of Impaired Wound Healing: A Systematic Review. Antioxidants (Basel, Switzerland) 7, (2018).

60. Mittal, M., Siddiqui, M. R., Tran, K., Reddy, S. P. \& Malik, A. B. Reactive Oxygen Species in Inflammation and Tissue Injury. Antioxid. Redox Signal. 20, 1126-1167 (2014).

61. Madigan, M. C. et al. Xanthine Oxidoreductase Function Contributes to Normal Wound Healing. Mol. Med. 21, 313-22 (2015).

62. Boulieu, R., Bory, C., Baltassat, P. \& Gonnet, C. Hypoxanthine and xanthine levels determined by high-performance liquid chromatography in plasma, erythrocyte, and urine samples from healthy subjects: the problem of hypoxanthine level evolution as a function of time. Anal. Biochem. 129, 398-404 (1983).

63. Kock, B. R., Delvowc, $\beta$, Sigmund, M. \& Greiling, H. Oxypurines and allantoin in blood of patients with ischaemic diseascs A Comparative Stiidy of the Concentrations of Hypoxanthine, Xanthine, Uric Acid and Allantoin in the Peripheral Blood of Normals and Patients with Acute Myocardial Infarcti. Eur. J. Clin. Chem. Clin. Biochem 32, 837-842 (1994).

64. Turgan, N. et al. Urinary hypoxanthine and xanthine levels in acute coronary syndromes. Int. J. Clin. Lab. Res. 29, 162-5 (1999).

65. Mendelsohn, M. E., Abramson, D. H., Senft, S., Servodidio, C. A. \& Gamache, P. H. Uric acid in the aqueous humor and tears of retinoblastoma patients. J. AAPOS Off. Publ. Am. Assoc. Pediatr. Ophthalmol. Strabismus 2, 369-71 (1998). 
66. Frycak, P., Huskova, R., Adam, T. \& Lemr, K. Atmospheric pressure ionization mass spectrometry of purine and pyrimidine markers of inherited metabolic disorders. J. Mass Spectrom. 37, 1242-1248 (2002).

67. Samanidou, V. F., Metaxa, A. S. \& Papadoyannis, I. N. Direct simultaneous determination of uremic toxins: creatine, creatinine, uric acid, and xanthine in human biofluids by HPLC. J. Liq. Chromatogr. Relat. Technol. 25, 43-57 (2002).

68. Percival, S. L., McCarty, S., Hunt, J. A. \& Woods, E. J. The effects of pH on wound healing, biofilms, and antimicrobial efficacy. Wound Repair Regen. 22, 174-186 (2014).

69. Power, G. D. pH, Exudate Composition and Temperature Measurement in Wounds - A Systematic Review. (2016).

70. Mostafalu, P. et al. A toolkit of thread-based microfluidics, sensors, and electronics for 3D tissue embedding for medical diagnostics. Microsystems Nanoeng. 2, 16039 (2016).

71. https://www.empa.ch/web/s604/wound-healing-sensor

72. Chanmugam, A. et al. Relative Temperature Maximum in Wound Infection and Inflammation as Compared with a Control Subject Using Long-Wave Infrared Thermography. Adv. Skin Wound Care 30, 406-414 (2017)

73. Dini, V. et al. Correlation Between Wound Temperature Obtained With an Infrared Camera and Clinical Wound Bed Score in Venous Leg Ulcers. Wounds a Compend. Clin. Res. Pract. 27, 274-8 (2015).

74. Dargaville, T. R. et al. Sensors and imaging for wound healing: A review. Biosens. Bioelectron. 41, 30-42 (2013).

75. Hayes, T. R. \& Su, B. Wound dressings. Electrospinning Tissue Regen. 317-339 (2011). doi:10.1533/9780857092915.2.317

76. Milne, S. D. et al. A wearable wound moisture sensor as an indicator for wound dressing change: an observational study of wound moisture and status. Int. Wound J. 13, 1309-1314 (2016).

77. Farooqui, M. F. \& Shamim, A. Low Cost Inkjet Printed Smart Bandage for Wireless Monitoring of Chronic Wounds. Sci. Rep. 6, 28949 (2016).

78. Salvo, P. et al. Sensors and Biosensors for C-Reactive Protein, Temperature and $\mathrm{pH}$, and Their Applications for Monitoring Wound Healing: A Review. Sensors 17, 2952 (2017) 
79. Bills, J. D. et al. Pilot study to evaluate a novel three-dimensional wound measurement device. Int. Wound J. 13, 1372-1377 (2016).

80. Nixon, M. \& Moore, C. A Three-Dimensional Approach To Measuring, Imaging and Documenting Wounds Evidence-Based Wound Surveillance. (2014).

81. Wang, S. C. et al. Point-of-care wound visioning technology: Reproducibility and accuracy of a wound measurement app. PLoS One 12, 1-14 (2017).

82. Barber, S. 42 OstomyWound Management FEATURE A Clinically Relevant Wound Assessment Method to Monitor Healing Progression. Ostomy. Wound. Manage. 54, 42-49 (2008).

83. Samanidou, V. F., Metaxa, A. S. \& Papadoyannis, I. N. Direct simultaneous determination of uremic toxins: creatine, creatinine, uric acid, and xanthine in human biofluids by HPLC. J. Liq. Chromatogr. Relat. Technol. 25, 43-57 (2002).

84. Tan, K. et al. Facet dependent binding and etching: Ultra-sensitive colorimetric visualization of blood uric acid by unmodified silver nanoprisms. Biosens. Bioelectron. 59, 227-232 (2014).

85. Dungchai, W., Chailapakul, O. \& Henry, C. S. Use of multiple colorimetric indicators for paper-based microfluidic devices. Anal. Chim. Acta 674, 227-233 (2010).

86. He, Y. et al. Uricase-free on-demand colorimetric biosensing of uric acid enabled by integrated CoP nanosheet arrays as a monolithic peroxidase mimic. Anal. Chim. Acta 1021, 113-120 (2018).

87. Shi, W., Fan, H., Ai, S. \& Zhu, L. Pd nanoparticles supported on nitrogen, sulfurdoped three-dimensional hierarchical nanostructures as peroxidase-like catalysts for colorimetric detection of xanthine. RSC Adv. 5, 32183-32190 (2015).

88. Wang, X.-X., Wu, Q., Shan, Z. \& Huang, Q.-M. BSA-stabilized Au clusters as peroxidase mimetics for use in xanthine detection. Biosens. Bioelectron. 26, 36143619 (2011).

89. Martinez-Pérez, D., Ferrer, M. L. \& Mateo, C. R. A reagent less fluorescent sol-gel biosensor for uric acid detection in biological fluids. Anal. Biochem. 322, 238-42 (2003).

90. Porez-Bendfto, D., Gomez-Hens, A. \& Carmen Gutlerrez, M. NonenzymaticStopped-Flow FluorimetricMethod for Direct Determinationof Uric Acid in Serum and Urine. CLIN. CHEM 35, 230 (1989). 
91. Beckman, J. S., Parks, D. A., Pearson, J. D., Marshall, P. A. \& Freeman, B. A. A sensitive fluorometric assay for measuring xanthine dehydrogenase and oxidase in tissues. Free Radic. Biol. Med. 6, 607-15 (1989).

92. Kumar, V., Misra, N., Varshney, L., Paul, J. \& Dhanawade, B. R. Enzyme immobilization on radiation grafted functional polymer support for Ag-Nanoparticle based optical biosensor Applications of ionizing radiation in AOP based degradation of persistent pollutants from aqueous streams View project Advanced Oxidation Pro. (2014). doi:10.1016/j.polymer.2014.04.012

93. Kiser, E. J., Johnson, G. F. \& Witte, D. L. Serum Uric Acid Determined by Reversed-Phase Liquid Chromatography with Spectrophotometric Detection. Clin. Chem. 24, 536 (1978).

94. Khajehsharifi, H. \& Pourbasheer, E. Simultaneous spectrophotometric determination of xanthine, hypoxanthine and uric acid in real matrix by orthogonal signal correction-partial least squares. J. Iran. Chem. Soc. 8, 1113-1119 (2011).

95. Newaz, M. A. \& Adeeb, N. N. Detection of Xanthine Oxidase In Human Plasma. Med J Malaysia 53, 70-75 (1998).

96. Devi, R., Thakur, M. \& Pundir, C. S. Construction and application of an amperometric xanthine biosensor based on zinc oxide nanoparticlesâ€"polypyrrole composite film. Biosens. Bioelectron. 26, 3420-3426 (2011).

97. Devi, R., Yadav, S. \& Pundir, C. Au-colloids-polypyrrole nanocomposite film based xanthine biosensor. Colloids Surfaces A Physicochem. Eng. Asp. 394, 38-45 (2011).

98. Jiang, M. \& Chen, J.-S. A Lable-free ECL Biosensor for The Detection of Uric Acid Based on Au NRs@TiO 2 Nanocomposite. Int. J. Electrochem. Sci 14, 2333-2344 (2019).

99. Yu, J., Ge, L., Huang, J., Wang, S. \& Ge, S. Microfluidic paper-based chemiluminescence biosensor for simultaneous determination of glucose and uric acid. Lab Chip 11, 1286 (2011).

100. Hlavay, J., Haemmerli, S. D. \& Guilbault, G. G. Fibre-optic biosensor for hypoxanthine and xanthine based on a chemiluminescence reaction. Biosens. Bioelectron. 9, 189-195 (1994).

101. Kwon, W., Kim, J. Y., Suh, S. \& In, M. K. Simultaneous determination of creatinine and uric acid in urine by liquid chromatography-tandem mass spectrometry with polarity switching electrospray ionization. Forensic Sci. Int. 221, 57-64 (2012). 
102. Ellerbe, P., Cohen, A., Welch, M. J. \& White, E. V. Determination of serum uric acid by isotope dilution mass spectrometry as a new candidate definitive method. Anal. Chem. 62, 2173-2177 (1990).

103. Rashed, M. S. et al. Determination of urinaryS-sulphocysteine, xanthine and hypoxanthine by liquid chromatography-electrospray tandem mass spectrometry. Biomed. Chromatogr. 19, 223-230 (2005).

104. Nakatani, A. et al. Xanthine oxidoreductase activity is associated with serum uric acid and glycemic control in hemodialysis patients. Sci. Rep. 7, 15416 (2017).

105. Cooper, N., Khosravan, R., Erdmann, C., Fiene, J. \& Lee, J. W. Quantification of uric acid, xanthine and hypoxanthine in human serum by HPLC for pharmacodynamic studies. J. Chromatogr. B 837, 1-10 (2006).

106. Jen, J.-F., Hsiao, S.-L. \& Liu, K.-H. Simultaneous determination of uric acid and creatinine in urine by an eco-friendly solvent-free high performance liquid chromatographic method. Talanta 58, 711-717 (2002).

107. Yong Zhao, F., Hua Wang, Z., Wang, H., Zhao, R. \& Yu Ding, M. Determination of uric acid in human urine by ion chromatography with conductivity detector. Chinese Chem. Lett. 22, 342-345 (2010).

108. RoyChoudhury, S. et al. (Invited) Multimodal Enzymatic Sensing for Continuous Wound Monitoring. ECS Trans. 88, 419-426 (2018).

109. Devi, R., Thakur, M. \& Pundir, C. S. Construction and application of an amperometric xanthine biosensor based on zinc oxide nanoparticlesâ€"polypyrrole composite film. Biosens. Bioelectron. 26, 3420-3426 (2011).

110. Devi, R., Yadav, S. \& Pundir, C. Au-colloids-polypyrrole nanocomposite film based xanthine biosensor. Colloids Surfaces A Physicochem. Eng. Asp. 394, 38-45 (2011)

111. Murugan, N., Chan-Park, M. B. \& Sundramoorthy, A. K. JES FOCUS ISSUE ON 4D MATERIALS AND SYSTEMS Electrochemical Detection of Uric Acid on Exfoliated Nanosheets of Graphitic-Like Carbon Nitride (g-C 3 N 4 ) Based Sensor. J. Electrochem. Soc. 166, 3163-3170 (2019).

112. Verma, S. et al. Uricase grafted nanoconducting matrix based electrochemical biosensor for ultrafast uric acid detection in human serum samples Biosensor View project Energy Efficient Opto-electronic Devices View project Uricase grafted nanoconducting matrix based electro. Int. J. Biol. Macromol. 130, 333-341 (2019).

113. Jiang, L., Gao, J., Huang, W., Chen, Z. \& Yi, C. Simultaneous Detection of Glucose, Uric Acid and Cholesterol Using Flexible Microneedle Electrode Array-based Biosensor and Multi-channel Portable Electrochemical Analyzer. Sensors Actuators B Chem. 287, 102-110 (2019). 
114. John, S. A. Simultaneous determination of uric acid and ascorbic acid using glassy carbon electrodes in acetate buffer solution. J. Electroanal. Chem. 579, 249-256 (2005).

115. Kassal, P. et al. Smart bandage with wireless connectivity for uric acid biosensing as an indicator of wound status. Electrochem. commun. 56, 6-10 (2015).

116. Sen, S. \& Sarkar, P. A novel third-generation xanthine biosensor with enzyme modified glassy carbon electrode using electrodeposited MWCNT and nanogold polymer composite film. $R S C A d v$. 5, 95911-95925 (2015).

117. Zen, J.-M., Lai, Y.-Y., Yang, H.-H. \& Kumar, A. S. Multianalyte sensor for the simultaneous determination of hypoxanthine, xanthine and uric acid based on a preanodized nontronite-coated screen-printed electrode. Sensors Actuators B Chem. 84, 237-244 (2002).

118. Spãtaru, N., Sarada, B. V., Tryk, D. A. \& Fujishima, A. Anodic Voltammetry of Xanthine, Theophylline, Theobromine and Caffeine at Conductive Diamond Electrodes and Its Analytical Application. Electroanalysis 14, 721 (2002).

119. Hammond, J. L., Formisano, N., Estrela, P., Carrara, S. \& Tkac, J. Electrochemical biosensors and nanobiosensors. Essays Biochem. 60, 69-80 (2016).

120. Esra Erden, P. \& K1lic, E. A review of enzymatic uric acid biosensors based on amperometric detection. Talanta 107, 312-323 (2013).

121. Numnuam, A., Thavarungkul, P. \& Kanatharana, P. An amperometric uric acid biosensor based on chitosan-carbon nanotubes electrospun nanofiber on silver nanoparticles. Anal. Bioanal. Chem. 406, 3763-3772 (2014).

122. Ali, M. et al. A paper based selective and quantitative detection of uric acid using citrate capped Pt nanoparticles (PtNPs) as a colorimetric sensing probe through a simple and remote based device (Supplementary Information). New J. Chem. 43, 7636-7645 (2019).

123. Alam, M. M., Asiri, A. M., Uddin, M. T., Islam, M. A. \& Rahman, M. M. Wetchemically prepared low-dimensional $\mathrm{ZnO} / \mathrm{Al}_{2} \mathrm{O}_{3} / \mathrm{Cr}_{2} \mathrm{O}_{3}$ nanoparticles for xanthine sensor development using an electrochemical method. RSC Adv. 8, 1256212572 (2018).

124. Yan, Z. et al. A novel colorimetric method based on copper nanoclusters with intrinsic peroxidase-like for detecting xanthine in serum samples. J. Nanoparticle Res. 19, 235 (2017).

125. El-Ansary, A. \& Faddah, L. M. Nanoparticles as biochemical sensors. Nanotechnol. Sci. Appl. 3, 65-76 (2010). 
126. Salahandish, R. et al. Sandwich-structured nanoparticles-grafted functionalized graphene based 3D nanocomposites for high-performance biosensors to detect ascorbic acid biomolecule. Sci. Rep. 9, 1226 (2019).

127. Rahman, M. M., Balkhoyor, H. B. \& Asiri, A. M. Ultra-sensitive xanthine sensor development based on wet-chemically prepared $\mathrm{Co} / \mathrm{ZnO}$ nanoparticles. Mater. Express 7, 93-103 (2017).

128. Wu, W., Min, H., Wu, H., Ding, Y. \& Yang, S. Electrochemical Determination of Uric Acid Using a Multiwalled Carbon Nanotube Platinum-Nickel Alloy Glassy Carbon Electrode. Anal. Lett. 50, 91-104 (2017).

129. Singh, B., Laffir, F., Dickinson, C., McCormac, T. \& Dempsey, E. Carbon Supported Cobalt and Nickel Based Nanomaterials for Direct Uric Acid Determination. Electroanalysis 23, 79-89 (2011).

130. Peng, B. et al. $\mathrm{CeO}_{2-\mathrm{x}} / \mathrm{C} / \mathrm{rGO}$ nanocomposites derived from Ce-MOF and graphene oxide as a robust platform for highly sensitive uric acid detection. Nanoscale $\mathbf{1 0}$, 1939-1945 (2018).

131. Wu, W.-C. et al. Nitrogen-doped carbon nanodots prepared from polyethylenimine for fluorometric determination of salivary uric acid. Microchim. Acta 186, 166 (2019).

132. Cai, W., Lai, J., Lai, T., Xie, H. \& Ye, J. Controlled functionalization of flexible graphene fibers for the simultaneous determination of ascorbic acid, dopamine and uric acid. Sensors Actuators B Chem. 224, 225-232 (2016).

133. Li, S.-M. et al. Fabrication of a silver nanowire-reduced graphene oxide-based electrochemical biosensor and its enhanced sensitivity in the simultaneous determination of ascorbic acid, dopamine, and uric acid. J. Mater. Chem. C 3, 94449453 (2015).

134. Fu, L. et al. An Electrochemical Sensor Based on Reduced Graphene Oxide and $\mathrm{ZnO}$ Nanorods-Modified Glassy Carbon Electrode for Uric Acid Detection. Arab J Sci Eng 41, 135-141 (2016).

135. Lu, Q. et al. One-step electrochemical synthesis of ultrathin graphitic carbon nitride nanosheets and their application to the detection of uric acid. Chem. Commun. 51, 12251-12253 (2015).

136. Dimcheva, N., Horozova, E. \& Jordanova, Z. An amperometric xanthine oxidase enzyme electrode based on hydrogen peroxide electroreduction. $Z$ Naturforsch $C 57$, 883-889 (2002). 
137. Anik, U. \& CUBUKCU, M. Examination of the Electroanalytic Performance of Carbon Nanotube (CNT) Modified Carbon Paste Electrodes as Xanthine Biosensor Transducers. Turk J Chem 32, 711-719 (2008).

138. Anik, Ü. \& Çevik, S. Double-walled carbon nanotube based carbon paste electrode as xanthine biosensor. Microchim. Acta 166, 209-213 (2009).

139. Li, Y., Schluesener, H. J., Xu, S. \& Org, W. G. Gold nanoparticle-based biosensors. Gold Bull. 43, 29-41 (2010).

140. Wang, J., Wang, L., Di, J. \& Tu, Y. Electrodeposition of gold nanoparticles on indium/tin oxide electrode for fabrication of a disposable hydrogen peroxide biosensor. Talanta 77, 1454-1459 (2009).

141. Dervisevic, M., Dervisevic, E., Çevik, E. \& Şenel, M. Novel electrochemical xanthine biosensor based on chitosan-polypyrrole-gold nanoparticles hybrid bionanocomposite platform. J. Food Drug Anal. 25, 510-519 (2017).

142. Çevik, S. Xanthine biosensor based on XO/AuNP/PtNP/MWCNT hybrid nanocomposite modified GCPE. Biotechnol. Bioprocess Eng. 21, 314-320 (2016).

143. Sher, M., Zhuang, R., Demirci, U. \& Asghar, W. Paper-based analytical devices for clinical diagnosis: recent advances in the fabrication techniques and sensing mechanisms. Expert Rev. Mol. Diagn. 17, 351-366 (2017).

144. Cruz, S. M. F., Rocha, L. A. \& Viana, J. C. Printing Technologies on Flexible Substrates for Printed Electronics. in Flexible Electronics (InTech, 2018). doi:10.5772/intechopen.76161

145. Bo, G., Ren, L., Xu, X., Du, Y. \& Dou, S. Recent progress on liquid metals and their applications. Adv. Phys. X 3, 412-442 (2018).

146. Castano, L. M., Flatau, A. \& Flatau, A. B. Smart fabric sensors and e-textile technologies: A review Orientation-dependent surface energy characterization of magnetostrictive metal alloys View project Smart fabric sensors and e-textile technologies: a review Topical Review Smart fabric sensors and. Smart Mater. Struct. 23, 27 (2014).

147. Kazani, I., Halili, A. \& Guxho, G. Nano-based Wearable Textile Sensors: A Review on Latest Developments. J. Nat. Tech. Sci. 43, 144-161 (2017).

148. Son, D., Koo, J. H., Lee, J. \& Kim, D.-H. High-Performance Wearable Bioelectronics Integrated with Functional Nanomaterials. in Stretchable Bioelectronics for Medical Devices and Systems 151-171 (Springer, Cham, 2016). doi:10.1007/978-3-319-28694-5_8 
149. Wang, Q. et al. Self-Healable Multifunctional Electronic Tattoos Based on Silk and Graphene. Adv. Funct. Mater. 29, 1808695 (2019).

150. Cruz, S. M. F., Rocha, L. A. \& Viana, J. C. Printing Technologies on Flexible Substrates for Printed Electronics. in Flexible Electronics (InTech, 2018). doi:10.5772/intechopen.76161

151. Yamanaka, K., Vestergaard, M. C. \& Tamiya, E. Printable Electrochemical Biosensors: A Focus on Screen-Printed Electrodes and Their Application. Sensors (Basel). 16, 1-16 (2016).

152. Svanholm, E. Printability and Ink-Coating Interactions in Inkjet Printing. (Karlstad University, 2007).

153. Palavesam, N. et al. Roll-to-roll processing of film substrates for hybrid integrated flexible electronics. Flex. Print. Electron. 3, 014002 (2018).

154. Subramanian, V. \& Sung, D. Gravure as an Industrially Viable Process for Printed Electronics. (2008).

155. Berenyi, R., Juhasz, G. \& Illyefalvi-Vitez, Z. Laser Manufacturing of Mechanical Structures in Flexible Substrates for Lifetime Increasing. in 2006 1st Electronic Systemintegration Technology Conference 1025-1031 (IEEE, 2006).

156. Colloc'h, N. et al. Crystal Structure of the protein drug urate oxidase-inhibitor complex at $2.05 \AA$ resolution. Nat. Struct. Mol. Biol. 4, 947-952 (1997).

157. Retailleau, P. et al. Complexed and ligand-free high-resolution structures of urate oxidase (Uox) from Aspergillus flavus : a reassignment of the active-site binding mode. Acta Crystallogr. Sect. D Biol. Crystallogr. 60, 453-462 (2004).

158. Poupon, A. \& Mornon, J.-P. Populations of hydrophobic amino acids within protein globular domains: Identification of conserved "topohydrophobic" positions. Proteins Struct. Funct. Genet. 33, 329-342 (1998).

159. Gethin, G. The significance of surface $\mathrm{pH}$ in chronic wounds. Wounds 3, 52-56 (2007).

160. Bhawna \& Pundir C S. Fabrication of dissolved O 2 metric uric acid biosensor based on uricase bound to PVC membrane. J. Sci. Ind. Res. 69, 695-699 (2010).

161. Kan, J., Pan, X. \& Chen, C. Polyaniline-uricase biosensor prepared with template process. Biosens. Bioelectron. 19, 1635-1640 (2004). 
162. Çete, S., Yaşar, A. \& Arslan, F. An Amperometric Biosensor for Uric Acid Determination Prepared from Uricase Immobilized in Polypyrrole Film. Artif. Cells, Blood Substitutes, Biotechnol. 34, 367-380 (2006).

163. De Fá Tima Giarola, J., Mano, V. \& Cé Sar Pereira, A. Development and Application of a Voltammetric Biosensor Based on Polypyrrole/uricase/graphene for Uric Acid Determination. Electroanalysis 30, 119-127 (2018).

164. Zhao, C., Wan, L., Wang, Q., Liu, S. \& Jiao, K. Highly sensitive and selective uric acid biosensor based on direct electron transfer of hemoglobin-encapsulated chitosan-modified glassy carbon electrode. Anal. Sci. 25, 1013-7 (2009).

165. Boateng, J. S. et al. Wound Healing Dressings and Drug Delivery Systems: A Review. J. Pharm. Sci. 97, 2892-2923 (2008).

166. Lee, H.-C. et al. Development of Anodic Titania Nanotubes for Application in High Sensitivity Amperometric Glucose and Uric Acid Biosensors. Sensors 13, 1416114174 (2013).

167. Bolivar, J. M. et al. Coating of Soluble and Immobilized Enzymes with Ionic Polymers: Full Stabilization of the Quaternary Structure of Multimeric Enzymes. Biomacromolecules 10, 742-747 (2009).

168. Bonnet, F. Macromolecular Crystallization Controlled by Colloidal Interactions: The Case of Urate Oxidase. in Crystallization - Science and Technology (InTech, 2012). doi:10.5772/47963

169. Umasankar, Y. \& Ramasamy, R. P. Enhanced Electron Transfer in Enzymatic Bioelectrodes by a Poly(vinyl alcohol) $N$-Methyl-4(4'-formylstyryl) Pyridinium Methosulfate Acetal Cationic Polymer. ChemElectroChem 1, 1834-1839 (2014).

170. Mahler, H. R., Hubscher, G. \& Baum, R. Studies on uricase. I. Preparation, purification, and properties of a cuproprotein. J. Biol. Chem. 216, 625-41 (1955).

171. Andreescu, S., Barthelmebs, L. \& Marty, J.-L. Immobilization of acetylcholinesterase on screen-printed electrodes: comparative study between three immobilization methods and applications to the detection of organophosphorus insecticides. Anal. Chim. Acta 464, 171-180 (2002).

172. Zhou, J. et al. Preparation and Characterization of Electrospun Polyvinyl Alcoholstyrylpyridinium/ $\beta$-cyclodextrin Composite Nanofibers: Release Behavior and Potential Use for Wound Dressing. Fibers Polym. 17, 1835-1841 (2016).

173. Putzbach, W., Ronkainen, N., Putzbach, W. \& Ronkainen, N. J. Immobilization Techniques in the Fabrication of Nanomaterial-Based Electrochemical Biosensors: A Review. Sensors 13, 4811-4840 (2013). 
174. Iswantini, D., Nurhidayat, N., Trivadila \& Widiyatmoko, O. Activity and stability of uricase from Lactobacillus plantarum immobilizated on natural zeolite for uric acid biosensor. Pakistan J. Biol. Sci. PJBS 17, 277-81 (2014).

175. Wang, Y. et al. Electrochemical Sensors for Clinic Analysis. Sensors 8, 2043-2081 (2008).

176. Takahiro Nakaminami, Shin-ichiro Ito, Susumu Kuwabata, A. \& Yoneyama*, H. A Biomimetic Phospholipid/Alkanethiolate Bilayer Immobilizing Uricase and an Electron Mediator on an Au Electrode for Amperometric Determination of Uric Acid. Anal. Chem. 71, 4278-4283 (1999).

177. Cass, A. E. G. et al. Ferrocene-mediated enzyme electrode for amperometric determination of glucose. Anal. Chem. 56, 667-671 (1984).

178. Freire, P. T. C., Barboza, F. M., Lima, J. A., Melo, F. E. A. \& Filho, J. M. Raman Spectroscopy of Amino Acid Crystals. in Raman Spectroscopy and Applications (InTech, 2017). doi:10.5772/65480

179. Kurouski, D., Van Duyne, R. P. \& Lednev, I. K. Exploring Structure and Formation Mechanism of Amyloid Fibrils by Raman Spectroscopy. A Review. Analyst 140, 4967-4980 (2015).

180. Haider, S. et al. Raman microspectroscopy reveals long-term extracellular activity of chlamydiae. Mol. Microbiol. 77, 687-700 (2010).

181. Krishnan, K. \& Plane, R. A. Raman spectra of ethylenediaminetetraacetic acid and its metal complexes. J. Am. Chem. Soc. 90, 3195-3200 (1968).

182. K'Owino, I. O. \& Sadik, O. A. Impedance Spectroscopy: A Powerful Tool for Rapid Biomolecular Screening and Cell Culture Monitoring. Electroanalysis 17, 21012113 (2005).

183. Selvam, A. P. et al. Development and validation of an impedance biosensor for point-of-care detection of vascular cell adhesion molecule-1 toward lupus diagnostics. Futur. Sci. OA 3, FSO224 (2017).

184. Votyakova, T. V. \& Reynolds, I. J. Detection of hydrogen peroxide with Amplex Red: interference by NADH and reduced glutathione auto-oxidation. Arch. Biochem. Biophys. 431, 138-144 (2004).

185. Schuhmann, W., Ohara, T. J., Schmidt, H. L. \& Heller, A. Electron transfer between glucose oxidase and electrodes via redox mediators bound with flexible chains to the enzyme surface. J. Am. Chem. Soc. 113, 1394-1397 (1991). 
186. Degani, Y. \& Heller, A. Direct electrical communication between chemically modified enzymes and metal electrodes. I. Electron transfer from glucose oxidase to metal electrodes via electron relays, bound covalently to the enzyme. J. Phys. Chem. 91, 1285-1289 (1987).

187. Xu, Q. et al. Graphene/polyaniline/gold nanoparticles nanocomposite for the direct electron transfer of glucose oxidase and glucose biosensing. Sensors Actuators $B$ Chem. 190, 562-569 (2014).

188. Simic, M. G. \& Jovanovic, S. V. Antioxidation mechanisms of uric acid. J. Am. Chem. Soc. 111, 5778-5782 (1989).

189. Tran, M. C. Biosensors. (Chapman \& Hall, 1993).

190. Gagne, R. R., Koval, C. A. \& Lisensky, G. C. Ferrocene as an internal standard for electrochemical measurements. Inorg. Chem. 19, 2854-2855 (1980).

191. Borland, L. M. \& Michael, A. C. An Introduction to Electrochemical Methods in Neuroscience. Electrochemical Methods for Neuroscience (CRC Press/Taylor \& Francis, 2007).

192. Kamin, R. A. \& Wilson, G. S. Rotating ring-disk enzyme electrode for biocatalysis kinetic studies and characterization of the immobilized enzyme layer. Anal. Chem. 52, 1198-1205 (1980).

193. Shu, F. R. \& Wilson, G. S. Rotating ring-disk enzyme electrode for surface catalysis studies. Anal. Chem. 48, 1679-1686 (1976).

194. Ensafi, A. A., Arabzadeh, A. \& Karimi-Maleh, H. Simultaneous Determination of Dopamine and Uric Acid by Electrocatalytic Oxidation on a Carbon Paste Electrode Using Pyrogallol Red as a Mediator. Anal. Lett. 43, 1976-1988 (2010).

195. Mickelsen, O. \& Keys, A. The composition of sweat, with special reference to the vitamins. J. Biol. Chem 149, 479-490 (1943).

196. Badrakhan, C.-D. et al. The methanol method for the quantification of ascorbic acid and dehydroascorbic acid in biological samples. J. Biochem. Biophys. Methods 58, 207-218 (2004).

197. Shome, S. et al. Dopamine Regulates Angiogenesis in Normal Dermal Wound Tissues. PLoS One 6, e25215 (2011).

198. Kelley, E. E. et al. Hydrogen peroxide is the major oxidant product of xanthine oxidase. Free Radic. Biol. Med. 48, 493-8 (2010).

199. Ball, E. G. The Journal of biological chemistry. J. Biol. Chem. 128, 51-67 (1939). 
200. Xia, M., Dempski, R. \& Hille, R. The reductive half-reaction of xanthine oxidase. Reaction with aldehyde substrates and identification of the catalytically labile oxygen. J. Biol. Chem. 274, 3323-30 (1999).

201. Hernández-Ruiz, J., Arnao, M. B., Hiner, A. N., García-Cánovas, F. \& Acosta, M. Catalase-like activity of horseradish peroxidase: relationship to enzyme inactivation by H2O2. Biochem. J. 354, 107-14 (2001).

202. Maehly, A. C. The Assay of Catalases and Peroxidases. in Methods of Biochemical Analysis (ed. David Glick) 357-424 (John Wiley \& Sons, Ltd, 1954). doi:10.1002/9780470110171.ch14

203. Tian, J. et al. Topical Delivery of Silver Nanoparticles Promotes Wound Healing. ChemMedChem 2, 129-136 (2007).

204. Guinovart, T., Valdés-Ramírez, G., Windmiller, J. R., Andrade, F. J. \& Wang, J. Bandage-Based Wearable Potentiometric Sensor for Monitoring Wound $\mathrm{pH}$. Electroanalysis 26, 1345-1353 (2014).

205. Mostafalu, P. et al. Wireless Flexible Smart Bandage for Continuous Monitoring of Wound Oxygenation. IEEE Trans. Biomed. Circuits Syst. 9, 670-677 (2015).

206. Zhao, X., Cui, X., Di, L. \& Li, W. Determination of hypoxanthine and xanthine in hippocampus by HPLC method. J. Chinese Med. Mater. 25, 716-7 (2002).

207. Çubukçu, M., Timur, S. \& Anik, Ü. Examination of performance of glassy carbon paste electrode modified with gold nanoparticle and xanthine oxidase for xanthine and hypoxanthine detection. Talanta 74, 434-439 (2007).

208. Banks, C. E., Davies, T. J., Wildgoose, G. G. \& Compton, R. G. Electrocatalysis at graphite and carbon nanotube modified electrodes: edge-plane sites and tube ends are the reactive sites. Chem. Commun. 7, 829 (2005).

209. Nugent, J. M., Santhanam, K. S. V., Rubio, A. \& P. M. Ajayan. Fast Electron Transfer Kinetics on Multiwalled Carbon Nanotube Microbundle Electrodes. Nano Lett 1, 87-91 (2001).

210. Yuan, W. et al. The edge- and basal-plane-specific electrochemistry of a single-layer graphene sheet. Sci. Rep. 3, 2248 (2013).

211. RoyChoudhury, S. et al. Continuous Monitoring of Wound Healing Using a Wearable Enzymatic Uric Acid Biosensor. J. Electrochem. Soc. 165, B3168-B3175 (2018). 
212. Dumee, L. et al. Control of Porosity and Pore Size of Metal Reinforced Carbon Nanotube Membranes. Membranes (Basel). 1, 25-36 (2011).

213. Du, D., Wang, M., Qin, Y. \& Lin, Y. One-step electrochemical deposition of Prussian Blue-multiwalled carbon nanotube nanocomposite thin-film: preparation, characterization and evaluation for $\mathrm{H}_{2} \mathrm{O}_{2}$ sensing. J. Mater. Chem. 20, 1532-1537 (2010).

214. Chen, W., Cai, S., Ren, Q.-Q., Wen, W. \& Zhao, Y.-D. Recent advances in electrochemical sensing for hydrogen peroxide: a review. Analyst 137, 49-58 (2012).

215. Nugent, J. M., Santhanam, K. S. V., Rubio, A. \& P. M. Ajayan. Fast Electron Transfer Kinetics on Multiwalled Carbon Nanotube Microbundle Electrodes. Nano Lett 1, 87-91 (2001).

216. Fridovich, I. \& Handler, P. Xanthine Oxidase II. Studies of the active site. J. Biol. Chem 231, 899-911 (1958).

217. Veitch, N. C. Horseradish peroxidase: a modern view of a classic enzyme. Phytochemistry 65, 249-259 (2004).

218. Sanz, V., de Marcos, S. \& Galbán, J. Uric acid determination using uricase and the autotransducer molecular absorption properties of peroxidase. Anal. Chim. Acta 607, 211-218 (2008).

219. Tang, D., Yuan, R. \& Chai, Y. Ultrasensitive Electrochemical Immunosensor for Clinical Immunoassay Using Thionine-Doped Magnetic Gold Nanospheres as Labels and Horseradish Peroxidase as Enhancer. Anal. Chem. 80, 1582-1588 (2008).

220. Cushley, M. J. \& Holgate, S. T. Bronchodilator actions of xanthine derivatives administered by inhalation in asthma. Thorax 40, 176-9 (1985).

221. Ansari, A. A., Alhoshan, M., Alsalhi, M. S. \& Aldwayyan, A. S. Prospects of nanotechnology in clinical immunodiagnostics. Sensors (Basel). 10, 6535-81 (2010).

222. Talley, K. \& Alexov, E. On the pH-optimum of activity and stability of proteins. Proteins 78, 2699-706 (2010).

223. Keith F. Cutting. Wound exudate: composition and functions. Br. J. Community Nurs. 4-9 (2003).

224. http://www.chem.latech.edu/ deddy/chem121/Alcohols.htm. 
225. Bewig, K. W. \& Zisman, W. A. The Wetting of Gold and Platinum by Water. J. Phys. Chem. 69, 4238-4242 (1965).

226. Huang, C.-T., Chen, M.-L., Huang, L.-L. \& Mao, I.-F. Uric acid and urea in human sweat. Chin. J. Physiol. 45, 109-15 (2002).

227. Peterson, S. L., McDonald, A., Gourley, P. L. \& Sasaki, D. Y. Poly(dimethylsiloxane) thin films as biocompatible coatings for microfluidic devices: Cell culture and flow studies with glial cells. J. Biomed. Mater. Res. 72A, 10-18 (2005).

228. Trengove, N. J., Langton, S. R. \& Stacey, M. C. Biochemical analysis of wound fluid from nonhealing and healing chronic leg ulcers. Wound Repair Regen. 4, 234239 (1996).

229. Montain, S. J., Cheuvront, S. N. \& Lukaski, H. C. Sweat mineral-element responses during $7 \mathrm{~h}$ of exercise-heat stress. Int. J. Sport Nutr. Exerc. Metab. 17, 574-82 (2007).

230. von Schenck, H., Lennmarken, C. \& Larsson, J. The effect of a fat emulsion on some common clinical chemical analyses. Clin. Nutr. 8, 79-81 (1989).

231. Tate, S., Price, A. \& Harding, K. Dressings for venous leg ulcers How well do dressings work? BMJ 1-7 (2018). doi:10.1136/bmj.k1604

232. Briggs, M., Nelson, E. A. \& Martyn-St James, M. Topical agents or dressings for pain in venous leg ulcers. Cochrane Database Syst. Rev. (2012). doi:10.1002/14651858.CD001177.pub3

233. Fernandez, M. L., Stupar, D., Croll, T., Leavesley, D. \& Upton, Z. Xanthine Oxidoreductase: A Novel Therapeutic Target for the Treatment of Chronic Wounds? Adv. Wound Care 7, 95-104 (2018).

234. Sheehan, P., Jones, P., Caselli, A., Giurini, J. M. \& Veves, A. Percent change in wound area of diabetic foot ulcers over a 4-week period is a robust predictor of complete healing in a 12-week prospective trial. Diabetes Care 26, 1879-82 (2003).

235. Coerper, S., Beckert, S., Küper, M. A., Jekov, M. \& Königsrainer, A. Fifty percent area reduction after 4 weeks of treatment is a reliable indicator for healing - analysis of a single-center cohort of 704 diabetic patients. J. Diabetes Complications 23, 4953 (2009).

236. RoyChoudhury, S. et al. Uricase Based Enzymatic Biosensor for Non-invasive Detection of Uric Acid by Entrapment in PVA-SbQ Polymer Matrix. Electroanalysis 30, 2374-2385 (2018). 
237. Haghi, M., Thurow, K. \& Stoll, R. Wearable Devices in Medical Internet of Things: Scientific Research and Commercially Available Devices. Healthc. Inform. Res. 23, 4-15 (2017).

238. Doukas, C. \& Maglogiannis, I. Bringing IoT and Cloud Computing towards Pervasive Healthcare. in 2012 Sixth International Conference on Innovative Mobile and Internet Services in Ubiquitous Computing 922-926 (IEEE, 2012). doi:10.1109/IMIS.2012.26.

239. Dimitrov, D. V. Medical Internet of Things and Big Data in Healthcare. Healthc. Inform. Res. 22, 156 (2016).

240. Wang, Y.-W., Yu, H.-L. \& Li, Y. Internet of things technology applied in medical information. in 2011 International Conference on Consumer Electronics, Communications and Networks (CECNet) 430-433 (IEEE, 2011). doi:10.1109/CECNET.2011.5768647.

241. RoyChoudhury, S., Shah, A., Shah, I., Umasankar, Y. \& Bhansali, S. NanoComposite Enzymatic Xanthine Biosensor for Wound Diagnostics. in 2018 IEEE SENSORS 1-4 (IEEE, 2018). doi:10.1109/ICSENS.2018.8589952.

242. https://www.alimed.com/disposable-wound-measuring-guides.html

243. Monitoring wound severity and healing using an enzymatic electrochemical approach, ASSIST NERC NSF Site Visit, Miami, 2017-2018.

244. RoyChoudhury, S. et al. Nanocomposite Bienzymatic Sensor for Monitoring Xanthine in Wound Diagnostics. J. Electrochem. Soc. 166, B3295-B3301 (2019). 
VITA

\section{SOHINI ROYCHOUDHURY}

Born, Kolkata, India

2006-2010

Bachelor of Engineering in Electronics and Communication (B. E.) Sikkim Manipal Institute of Technology

Manipal University-Sikkim Campus

Sikkim, India

2014-2019

Doctoral Candidate

Electrical and Computer Engineering

Florida International University

Miami, Florida

\section{JOURNAL PUBLICATIONS and CONFERENCE PROCEEDINGS}

1. RoyChoudhury, S., Rawat, V., Jalal, A. H., Kale, S. N., and Bhansali, S., (2016). Recent advances in metamaterial split-ring-resonator circuits as biosensors and therapeutic agents. J. Biosensors and Bioelectronics, 86:595-608.

2. RoyChoudhury, S., Umasankar, Y., Jaller, J., Herskovitz, I., Mervis, J., Darwin, E., Hirt, P. A., Borda, L. J., Lev-Tov, H. A., Kirsner, R. S., and Bhansali, S., (2018). Continuous Monitoring of Wound Healing Using a Wearable Enzymatic Uric Acid Biosensor. J. Electrochem. Soc., 165 (8): B3168-B3175.

3. RoyChoudhury, S., Umasankar, Y., Hutcheson, J., Lev-Tov, H. A., Kirsner, R. S., and Bhansali, S., (2018). Uricase Based Enzymatic Biosensor for Non-invasive Detection of Uric Acid by Entrapment in PVA-SbQ Polymer Matrix. J. Electroan., $30: 2374-2385$.

4. Alam, F., RoyChoudhury, S., Jalal, A. H., Umasankar, Y., Forouzanfar, S., Akter, N., Bhansali, S., and Pala, N., (2018). Lactate biosensing: The emerging point-ofcare and personal health monitoring. J. Biosensors and Bioelectronics, 117:818829.

5. Jalal, A. H., Alam, F., RoyChoudhury, S., Umasankar, Y., Pala, N., and Bhansali, S., (2018). Prospects and Challenges of Volatile Organic Compound Sensors in Human Healthcare. J. ACS Sensors, 3:1246-1263.

6. RoyChoudhury, S., Umasankar, Y., Bhushan, P., Hirt, P. A., MacQuhae, F. E., Borda, L. J., Lev-Tov, H. A., Kirsner, R. S., and Bhansali, S., (2019). Nanocomposite Bienzymatic Sensor for Monitoring Xanthine in Wound Diagnostics. J. Electrochem. Soc., 166 (9) B3295-B3301. 
7. Bhushan, P., Umasankar, Y., RoyChoudhury, S., Hirt, P. A., MacQuhae, F. E., Borda, L. J., Lev-Tov, H. A., Kirsner, R. S., and Bhansali, S., (2019). Biosensor for Monitoring Uric Acid in Wound and its Proximity: A Potential Wound Diagnostic Tool. J. Electrochem. Soc., 166 (10) B830-B836.

8. RoyChoudhury, S., Manickam, P., Umasankar, Y., and Bhansali, S., (2015). Enzyme Functionalized Metal Nanostructures for Enhanced Electrochemical Sensing of Lactate, ECS Trans., 69 (37): 7-15.

9. RoyChoudhury, S., Manickam, P., Umasankar, Y., and Bhansali, S., (2015). Biosensor Device for Continuous Lactate Monitoring, Defense Innovation Technology Acceleration Challenges.

10. RoyChoudhury, S., Umasankar, Y., and Bhansali, S., (2017). A Wearable Electrochemical Sensor to Monitor Progression of Wound Healing, 232nd ECS Conf.

11. Pierre, M., RoyChoudhury, S., Umasankar, Y., Manickam, P., Madhu, S., Fernandez, R. E., Chinnuswamy, V., Munroe N., and Bhansali, S., (2017). Textile Fiber Electrode to Monitor Uric Acid as a Marker for Assessing Wound Chronicity, 232nd ECS Conf.

12. RoyChoudhury, S., Umasankar, Y., and Bhansali, S., (2018). Purine precursor detection for pre-symptomatic diagnosis of wounds, 255th ACS National Meeting.

13. RoyChoudhury, S., Shah, A. P., Shah, I., Umasankar, Y., and Bhansali, S., (2018). Nano-Composite Enzymatic Xanthine Biosensor for Wound Diagnostics, IEEE Sensors Xplore.

14. RoyChoudhury, S., Bhushan, P., Umasankar, Y., Hirt, P. A., Lev-Tov, H. A., Kirsner, R. S., and Bhansali, S., (2018). Multimodal Enzymatic Sensing for Continuous Wound Monitoring, ECS Trans. 88(1): 419-426. 